(c) Copyright 2014

Nishita Anandan 



\title{
Heat resistant polymer electrolyte for enhanced organic electrochromic windows based on poly (3,3-dimethyl-3, 4-dihydro-2H-thieno[3,4-b][1,4]dioxepine
}

\author{
Nishita Anandan
}

A thesis

submitted in partial fulfillment of the requirements for the degree of

Master of Science

University of Washington

2014

Committee:

Minoru Taya, Chair

Joyce Cooper

Sooyeun Kim

Program Authorized to Offer Degree: Mechanical Engineering 

University of Washington

\begin{abstract} poly (3,3-dimethyl-3, 4-dihydro-2H-thieno[3,4-b][1,4]dioxepine

\author{
Nishita Anandan \\ Chair of the Supervisory Committee: \\ Professor Minoru Taya \\ Department of Mechanical Engineering
}

Heat resistant polymer electrolyte for enhanced organic electrochromic windows based on

Electrochromic Windows(ECWs) have the potential to save energy through dynamic control of light and solar energy entering a room (via solar heat gain coefficient control). ECWs have been developed as an optical shutter in airplane, building and automobile applications. An ECW is composed of three components, a working electrode based on electrochromic materials, a counter electrode based on ion storage materials and the electrolyte as an ionic conducting layer. Organic ECWs have been gaining popularity due to easy and cost effective manufacturing, availability of wide range of colors, high optical contrast and flexibility in design. However there are challenges in commercialization and application of organic ECWs. The application of ECWs as a sunroof in automobiles demands operation in harsh environment conditions like elevated temperature. Consequently the University of Washington, Center for Intelligent Materials and Systems has been developing a heat resistant organic ECW that can be operated at elevated temperatures maintaining high optical contrast, fast switching speed, optical color memory and electrochemical stability. The proposed design is an ECW based on poly (3,3-dimethyl-3,4-dihydro-2H-thieno[3,4b] $[1,4]$ dioxepine),PPRODOT- $\mathrm{Me}_{2}$ as a working electrode, $\mathrm{V}_{2} \mathrm{O}_{5}-\mathrm{TiO}_{2}$ composite materials as a counter electrode and poly(ethylene imine) based electrolyte. The ionic conductivity of the electrolyte was calculated through complex impedance method and temperature dependence of the electrolyte was determined using environment test chamber to control a 
temperature range of $15^{\circ}$ to $80^{\circ}$ Celsius for 100 hours. A $76 \times 76 \mathrm{~mm}^{2}$ ECW was developed and the optical transmittance change was observed by Chronoamperomerty and Time course measurement. The developed electrochromic window showed good optical contrast, electrochemical stability and fast response time after testing at elevated temperatures for 100 hours. 


\section{TABLE OF CONTENTS}

Page

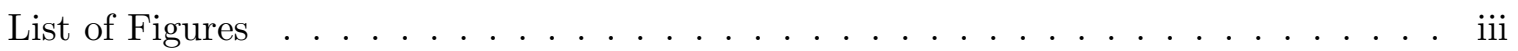

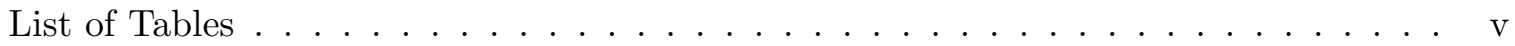

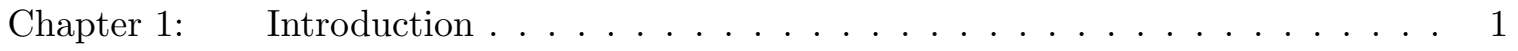

1.1 Electrochromic Windows $(\mathrm{ECWs}) \ldots \ldots \ldots \ldots \ldots \ldots$

1.2 Inorganic ECWs . . . . . . . . . . . . . . . . . . 3

1.3 Organic ECWs . . . . . . . . . . . . . . . . . . 7

1.3 .1 Viologen systems . . . . . . . . . . . . . . . . . 9

1.3.2 Conjugated electrochromic polymers . . . . . . . . . . . . 12

1.4 Organometallic ECW . . . . . . . . . . . . . . . . . 17

Chapter 2: Applications of Electrochromic Windows . . . . . . . . . . . 19

2.1 Electrochromic windows as sunroof in automobiles . . . . . . . . . . . 20

2.1 .1 Problem Statement . . . . . . . . . . . . . . . . . . . . . . . . . . . 22

2.1.2 Components of ECW . . . . . . . . . . . . . 23

Chapter 3: Electrolyte development . . . . . . . . . . . . . . 35

3.1 Development of electrolyte for ECWs . . . . . . . . . . . . . . . 35

3.2 Ionic conductivity of electrolyte . . . . . . . . . . . . . . 35

3.3 Polyethylene Imine $(\mathrm{PEI})$ based electrolyte . . . . . . . . . . . . . . . 37

3.3.1 Preparation of heat resistant PEI based electrolytes . . . . . . . . . 39

Chapter 4: Development of heat resistant ECW . . . . . . . . . . 58

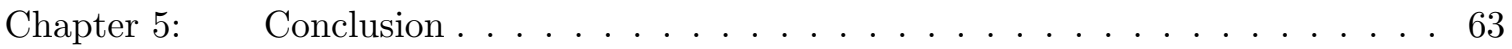

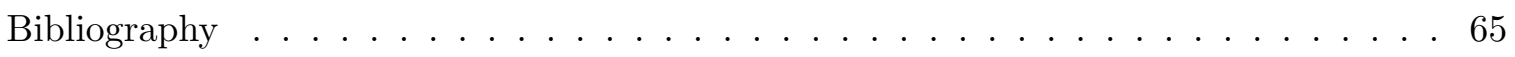

Appendix A: Experimental results: Variation of Transmittance of electrolytes from $20^{\circ} \mathrm{C}$ to $120^{\circ} \mathrm{C} \ldots \ldots \ldots \ldots \ldots 71$ 
Appendix B: Experimental results: Variation of Ionic Conductivity of electrolytes from $20^{\circ} \mathrm{C}$ to $120^{\circ} \mathrm{C} \ldots \ldots \ldots$. . . . . . . . . . . . . . . .

Appendix C: Experimental results: Counter Electrode Transmittance . . . . . . . . 91

Appendix D: Experimental results: Working Electrode Transmittance . . . . . . . . 94 


\section{LIST OF FIGURES}

Figure Number $\quad$ Page

1.1 Basic design of electrochromic windows with cations moving towards the electrochromic layer under applied voltage $[3] \ldots \ldots$. . . . . . . . 2

1.2 (a) Setup of WONWS-RGO composite-based EC system, (b) schematically structure illustrating the electrochromism of WONWS-RGO composite, and

(c) crystal structure of monoclinic $W_{18} O_{49}$ containing different tunnels that can accommodate $L i^{+}$ions[14]. . . . . . . . . . . . . . . 8

1.3 Three common redox states of viologen[31] . . . . . . . . . . . . . . . 10

1.4 Proposed mechanism of electropolymerization of pyrrole. Example for radicalcation/radical-cation coupling[28]. . . . . . . . . . . . . . . . . 12

1.5 Polypyrole exhibiting electrochromism [34]. . . . . . . . . . . . . . . . 13

1.6 Spectroelectrochemistry for PEDOT film on ITO[36] . . . . . . . . . . . . . 15

1.7 Synthesis of ProdDOT-Me $2[34] \ldots \ldots \ldots \ldots \ldots \ldots$

2.1 Heating curves for the air inside a parked car under different conditions [45] . 21

2.2 Short wavelengths of visible light are readily transmitted through thetransparentwindshield. [46] . . . . . . . . . . . . . . . . 22

2.3 Working of ECW, positive voltage applied PProDOT-Me2 film. The film is oxidized and turns light blue with high transmittance of $71 \%$. . . . . . . . . 24

2.4 Working of ECW, negative voltage applied PProDOT-Me2 film. The film is reduced and turns dark blue with transmittance less than 5\%. . . . . . . . 25

2.5 ProDOT- $-\mathrm{Me}_{2}$ structure in a) neutral and b) oxidized states[?] . . . . . . . . . 26

2.6 As the conjugation length increases from ethylene to polyacetylene the band gap decreases. . . . . . . . . . . . . . . . . . . 27

2.7 Variation of transmittance of PProDOT-Me $\mathrm{M}_{2}$ film from $20{ }^{\circ} \mathrm{C}$ to $120{ }^{\circ} \mathrm{C}$ in the oxidized and neutral state. . . . . . . . . . . . . . . . 31

2.8 Variation of transmittance of PProDOT-Me $\mathrm{Me}_{2}$ film from $20{ }^{\circ} \mathrm{C}$ to $120{ }^{\circ} \mathrm{C}$ in the oxidized and reduced state. . . . . . . . . . . . . . . . 34

3.1 Schematic representation of cells used for ionic conductivity measurement by impedance method as an example. (a) Solid-state samples; (b) liquid-state samples[55] . . . . . . . . . . . . . . . . . . 36 
3.2 Variation of transmittance of electrolytes 1 to 8 at $20^{\circ} \mathrm{C}, 40{ }^{\circ} \mathrm{C}, 60{ }^{\circ} \mathrm{C}, 80$ ${ }^{\circ} \mathrm{C}, 100{ }^{\circ} \mathrm{C}$ and $120{ }^{\circ} \mathrm{C}$. . . . . . . . . . . . . . . . . 42

3.3 Stability of ionic conductivity of electrolytes 1 to 8 at $20^{\circ} \mathrm{C}, 40{ }^{\circ} \mathrm{C}, 60{ }^{\circ} \mathrm{C}$, $80{ }^{\circ} \mathrm{C}, 100{ }^{\circ} \mathrm{C}$ and $120^{\circ} \mathrm{C} \ldots \ldots \ldots \ldots \ldots$. . . . . . . . . . . . . 44

3.4 DSC measurements of electrolytes 1 to 8 from $20^{\circ} \mathrm{C}$ to $130{ }^{\circ} \mathrm{C} \ldots \ldots$. . . 45

3.5 Structure of DGEBA $[66] \ldots \ldots \ldots \ldots \ldots \ldots$

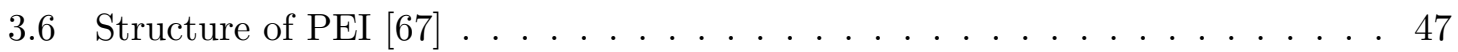

3.7 Epoxide-amine structure $[60] \ldots \ldots \ldots \ldots \ldots$

3.8 Variation of transmittance of electrolytes 9 to 16 at $20{ }^{\circ} \mathrm{C}, 40{ }^{\circ} \mathrm{C}, 60{ }^{\circ} \mathrm{C}, 80$

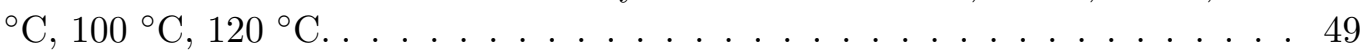

3.9 Stability of ionic conductivity of electrolytes 9 to 16 at $20^{\circ} \mathrm{C}, 40{ }^{\circ} \mathrm{C}, 60{ }^{\circ} \mathrm{C}$, $80{ }^{\circ} \mathrm{C}, 100{ }^{\circ} \mathrm{C}, 120{ }^{\circ} \mathrm{C} \ldots \ldots \ldots \ldots \ldots \ldots$

3.10 DSC measurements of electrolytes 9 to 16 from $20{ }^{\circ} \mathrm{C}$ to $130{ }^{\circ} \mathrm{C}$. . . . . . . 51

3.11 Structure of PEGMA $[68] \ldots \ldots \ldots \ldots \ldots \ldots$

3.12 Structure of PEG $200[69] \ldots \ldots \ldots \ldots \ldots \ldots$

3.13 Structure of DMPA $[70] \ldots \ldots \ldots \ldots \ldots$. . . . . . . . . . . . 54

3.14 Variation of transmittance of electrolytes 17 to 20 at $20^{\circ} \mathrm{C}, 40^{\circ} \mathrm{C}, 60{ }^{\circ} \mathrm{C}, 80$ ${ }^{\circ} \mathrm{C}, 100{ }^{\circ} \mathrm{C}, 120^{\circ} \mathrm{C} \ldots \ldots \ldots \ldots \ldots \ldots \ldots 5$

3.15 Stability of ionic conductivity of electrolytes 17 to 20 at $20^{\circ} \mathrm{C}, 40{ }^{\circ} \mathrm{C}, 60{ }^{\circ} \mathrm{C}$, $80{ }^{\circ} \mathrm{C}, 100{ }^{\circ} \mathrm{C}, 120{ }^{\circ} \mathrm{C} \ldots \ldots \ldots \ldots \ldots \ldots$

3.16 DSC measurements of electrolytes 17 to 20 from $20{ }^{\circ} \mathrm{C}$ to $130{ }^{\circ} \mathrm{C}$. . . . . . . 57

4.1 Time course measurement of ECWs with electrolytes 3, 4 and 5 . These electrolytes have lower PEI/DMSO weight ratio. . . . . . . . . . . . . . 59

4.2 Time course measurement of ECWs with electrolytes 8, (a) before the heat treatment, (b) after the heat treatment. . . . . . . . . . . . 6 60

4.3 Time course measurement of ECWs with electrolyte 10 (a) Before the heat treatment, (b) after the heat treatment at $80^{\circ} \mathrm{C}$ for 100 hours, (c) after the heat treatment at $120^{\circ} \mathrm{C}$ for 30 minutes. . . . . . . . . . . . . . . . 61

4.4 Time course measurement of ECWs with electrolyte 11 (a) Before the heat treatment, (b) after the heat treatment. . . . . . . . . . . . . 62

4.5 Time course measurement of ECWs with electrolyte 18 (a) Before the heat treatment, (b) after the heat treatment at $80^{\circ} \mathrm{C}$ for 100 hours, (c) after the heat treatment at $120^{\circ} \mathrm{C}$ for 30 minutes. . . . . . . . . . . . 62 


\section{LIST OF TABLES}

Table Number $\quad$ Page

1.1 Composition and properties of electrochromic oxides, $[3] \ldots \ldots \ldots$

3.1 Weight ratio of PEI, DMSO and $\mathrm{LiClO}_{4}$ or $\mathrm{LiCF}_{3} \mathrm{SO}_{3}$ in developed electrolytes. 40

3.2 Weight ratio of PEI, DMSO, $\mathrm{LiClO}_{4}$ and DGEBA in developed electrolytes. . 46

3.3 Weight ratio of PEI, DMSO, $\mathrm{LiClO}_{4}$, PEGMA, PEG 200 and DMPA in developed electrolytes . . . . . . . . . . . . . . . . 52 


\section{ACKNOWLEDGMENTS}

This study was conducted under a grant from National Science Foundation to University of Washington (NSF-EFRI 1038165), PI : Dr. Taya, Co-PIs: Dr. Cooper, Dr. Luscombe, Dr. Kuga and Argil Inc., sample based research seed fund. I would like to thank my committee members Dr.Taya, Dr. Cooper and Dr. Kim for providing the opportunity to work on this project, and for their continuous guidance and support. I would like to acknowledge Rodriguez Pham, Hiromi Yasuda and Dr.Keishi Naito, colleagues in Center for Intelligent Materials and Systems at University of Washington, who assisted me through my Masters research. Lastly, I would like to express special gratitude to Rahul Warrier, my fiancé for being my biggest pillar of strength and support in completing this project and thesis. 


\section{DEDICATION}

to my parents 



\section{Chapter 1}

\section{INTRODUCTION}

\subsection{Electrochromic Windows (ECWs)}

An electrochromic window (ECW) is a device which changes its optical properties in a reversible and persistent way under the action of a voltage pulse. ECWs have significant potential to reduce energy use in buildings. Buildings account for $\approx 40 \%$ of the worlds energy use with the resulting carbon emissions substantially more than those in the transportation sector[1]. In the US, the energy lost through todays inefficient window stock accounts for $\approx 30 \%$ of building heating and cooling energy[2]. ECWs can be a significant factor in reducing building energy use and ultimately in achieving net zero energy buildings. Dynamic windows are key to achieving this goal while preserving the view and enhancing the comfort and productivity of knowledge workers.

There are several possible configurations for ECWs but the most practical one is shown in Figure 1.1. ECWs contain seven layers, the outermost being the glass substrate on which transparent conductive oxide film is coated. The conductive layer is required to be transparent to enhance the optical performance of the ECW. The electrochromic and ion storage layer is now coated on the conductive oxide. Finally the electrolyte is sandwiched between the electrochromic and the ion storage layer. The assembled device is sealed with an appropriate adhesive and tested for optical performance. The electrochromic material has mixed conductivity for both electrons and ions, if ions are introduced from electrolyte or from an adjacent ion conductor there is a corresponding charge-balancing counter flow of electrons from the transparent electron conductor. The electrons remain in the electrochromic film as long as the ions reside there and, the electrons will then evoke persistent change of the optical properties. Depending on the nature of electrochromic film used the 
injected electron may increase or decrease transparency. The electrolyte can be a thin film or bulk material like solid inorganic or organic polymeric material. Lastly, the ion storage material provides cyclic stability to the ECW by maintaining ions for the next cycle. The ion storage material should also have high ionic and electronic conductivity as the electrochromic material [3]. The ion storage material may or may not have electrochromic properties. When a voltage is applied between the transparent conductors as described in Figure 1.1, a distributed electrical field is set up and ions are moving in and out of electrochromic film causing change in their optical property. The charge-balancing counterflow of electrons through the external circuit then leads to a variation of electron density in the electrochromic material thereby result in modulation of their optical properties. If the ion conductor has negligible electronic conductivity, the device will exhibit open circuit memory, so that the optical properties remain stable over periods of time. The applied voltage to the ECW should be of the order of few volts only since higher voltages may lead to rapid deterioration of the device[3].

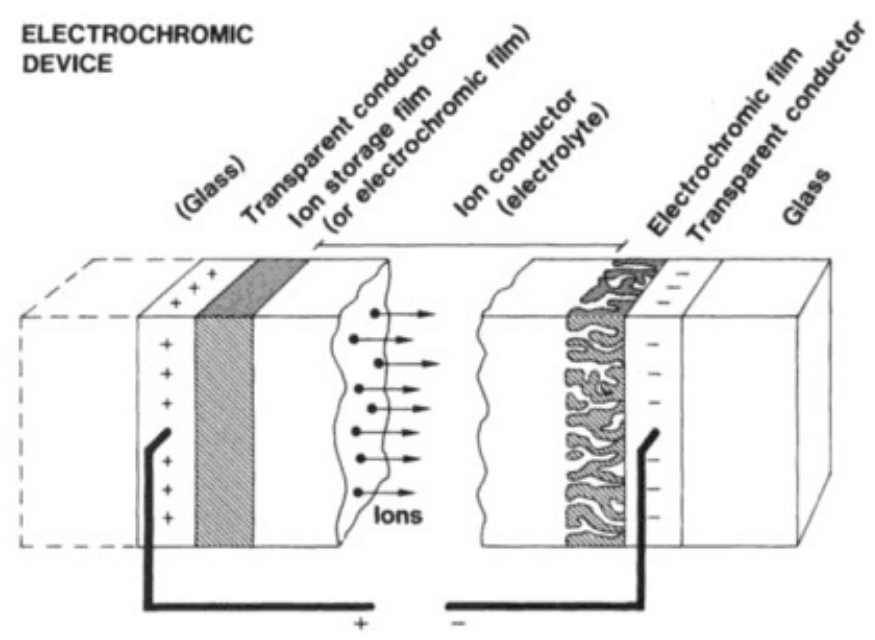

Figure 1.1: Basic design of electrochromic windows with cations moving towards the electrochromic layer under applied voltage [3] 


\subsection{Inorganic $\mathrm{ECWS}$}

Oxides of some transition metals show electrochromism. Cathodic coloration is found in oxides of Titanium, Niobium, Molybdenum, Tantalum and Tungsten of which Tungsten oxide is most widely used. Whereas, anodic coloration was found in oxides of Chromium, Manganese, Nickel, Iron, Cobalt and Iridium with Nickel and Iridium oxide being the most popular electrochromic materials. Vanadium pentoxide is exceptional since it exhibits both anodic and cathodic coloration within different wavelength ranges whereas Vanadium dioxide exhibits anodic electrochromism. Oxides of Titanium, Nickel, Molybdenum, Tantalum, Tungsten and Iridium had the capacity to be fully transparent to visible light whereas other oxides have residual absorption either across the entire visible range or in the blue part of the spectrum[3].

Most of the electrochromic oxides reported are oxides of transient metals which implies that electronic structure plays an important role in electrochromism. It is important to note that for oxides based on Iron, Nickel and Cobalt the Hydrogen plays a critical part by stabilizing a favorable structure. All electrochromic oxides are constructed from one type of building blocks, viz. $\mathrm{MeO}_{6}$ octahedra with a central transition metal (Me) atom surrounded by six equidistant oxygen atoms. The building blocks are connected either by corner-sharing or by a combination of cornersharing and edge sharing, and many different crystal structures are known. These structures can be divided into three dimensional framework (F) structures and weakly coupled, essentially two dimensional layer (L) structures [3].

The electrochromic oxides are permeable to ions and also have reasonable electrical conductivity. The ions can move and reside in spaces between the $\mathrm{MeO}_{6}$ octahedra. However, for the framework structures these spaces is not large enough to yield reasonable intercalation/deintercalation rates and therefore the materials must have fine grained character with low density grain boundaries. Extended columnar configuration that allows easy transport of ions over the cross-section of the electrochromic film is also desirable. These columns further open up the structure and provides conduits and intercalation sites for the ions. 


\begin{tabular}{lccc}
\hline \hline Oxide & Coloration & $\begin{array}{c}\text { Full } \\
\text { transp. }\end{array}$ & $\begin{array}{c}\text { Structure } \\
\text { type }\end{array}$ \\
\hline $\mathrm{TiO}_{2}$ & $\mathrm{C}$ & $\mathrm{Y}$ & $\mathrm{F}$ \\
$\mathrm{V}_{2} \mathrm{O}_{5}$ & $\mathrm{C} / \mathrm{A}$ & $\mathrm{N}$ & $\mathrm{L}^{1}$ \\
$\mathrm{Cr}_{2} \mathrm{O}_{3}$ & $\mathrm{~A}$ & $\mathrm{~N}$ & $\mathrm{~F}$ \\
$\mathrm{MnO}_{2}$ & $\mathrm{~A}$ & $\mathrm{~N}$ & $\mathrm{~F}$ \\
$\mathrm{FeO}_{2}$ & $\mathrm{~A}$ & $\mathrm{~N}$ & $\mathrm{~F}$ \\
$\mathrm{CoO}_{2}$ & $\mathrm{~A}$ & $\mathrm{~N}$ & $\mathrm{~L}$ \\
$\mathrm{NiO}_{2}$ & $\mathrm{~A}$ & $\mathrm{Y}$ & $\mathrm{L}$ \\
$\mathrm{Nb}_{2} \mathrm{O}_{5}$ & $\mathrm{C}$ & $\mathrm{Y}$ & $\mathrm{F}$ \\
$\mathrm{MoO}_{3}$ & $\mathrm{C}$ & $\mathrm{Y}$ & $\mathrm{F} / \mathrm{L}$ \\
$\mathrm{RhO}_{2}$ & $\mathrm{~A}$ & $?$ & $\mathrm{~F}$ \\
$\mathrm{Ta}_{2} \mathrm{O}_{5}$ & $\mathrm{C}$ & $\mathrm{Y}$ & $\mathrm{F}$ \\
$\mathrm{WO}_{3}$ & $\mathrm{C}$ & $\mathrm{Y}$ & $\mathrm{F}$ \\
$\mathrm{IrO}_{2}$ & $\mathrm{~A}$ & $\mathrm{Y}$ & $\mathrm{F}$ \\
& & & \\
\hline \hline
\end{tabular}

Table 1.1: Composition and properties of electrochromic oxides, [3] 
Of all the single oxide electrochromic materials discussed Tungsten oxide is the most popular due to its capacity to be fully transparent, easy preparation, fast color switching speed, high coloration efficiency and ideal cyclic stability[13]. In recent years researchers are putting in considerable effort in the development of $1 \mathrm{D}$ tungsten oxide nanomaterials(WONMS) since they exhibit enhanced electrochromic properties compared with their bulk counterpart form due to larger surface to volume ratio, open structures and short diffusion length of ions[14]. Series of 1D tungstun oxide nanostructures as electrochromic materials have been prepared till now, such as $\mathrm{WO}_{3}$ nanowires[15], $\mathrm{WO}_{3}$ nanowires arrays[16], $\mathrm{WO}_{3}$ nanowires plates[17] etc. For electrochromic application, the 1D WONMs need to be re-constructed as EC films on conductive substrates. During the preparation of EC films, however, the 1D WONMs prefer to aggregate or stack and form large structures, which would reduces their interfacial contact area with the electrolyte and hinder the ion diffusion in the EC films. Therefore, it is still challenge to take full advantages of the 1D WONMs as EC materials.

Conductive substrates to grow the nanoparticles is essential to the use of EC nanoparticles. Recently, graphene and its derivatives such as graphene oxide (GO) and chemically reduced graphene oxide (RGO) have emerged as new promising platforms for the design of a diversity of electronic devices[18]. In particular, the GO sheets, which possess many oxygenated functional groups, provide chemically reactive sites for anchoring functional inorganic oxide semiconductors[19]. Under well controlled synthetic conditions, the oxide semiconductors can be strongly coupled with the GO sheets to form novel hybrid composite, while the GO sheets can be simultaneously reduced to RGO sheets [20]. In addition, the interaction between the inorganic precursors and the GO supports could realize in situ growth of lowdimensional oxide semiconductor nanostructures on the GO sheets with controllable morphology, size, structure, dispersion, and coverage density [21]. In spite of lower electrical conductivity and slower carrier mobility than graphene, the RGO sheets could also significantly enhance functionalities of the inorganic oxide semiconductors. Up to now, the RGO sheets have been widely composited with diverse oxide semi- 
conductors and they have found many potential applications in supercapacitors[22], Li ion battery[23] etc. Since the EC performance depends strongly on the diffusion of ions in the EC materials]. Due to the large specific surface area, open 2D permeable channels, a certain degree of electrical conductivity, and the ability to anchor other inorganic materials, the RGO sheets would certainly facilitate the ion diffusion and accelerate the charge transfer in the EC device[24]. Therefore, it can be predicted that, the distinctive EC performance of the 1D WONMs could be strengthened through the incorporation with the RGO sheets.

Recently, Chang et al have grown Tungsten oxide on GO sheets to develop high performance electrochromic material [29]. In this study, the synthesis of hybrid electrochromic composite through nucleation and growth of ultrathin tungsten oxide nanowires on GO sheets using a facile solvothermal route is discussed. GO sheets form an ideal template for the growth of oxide semiconductors on their surfaces or edges since they contain abundant of oxygenated functional groups. The oxygencontaining functional groups serve as favourable nucleation and anchor sites for the in situ growth of tungsten oxide nanocrystals on the GO sheets owing to their coordination with $\mathrm{W}(\mathrm{VI})$ ions. In this case GO sheets were effective supports for the growth of WONMs. The WONMs were formed on the GO sheets and were well dispersed. There are no extra WONMs protruding from the GO sheets or remained in the solution proving that nucleation and growth of the WONMs are aided by oxygenated functional groups on the surface. The competition between the growth of tungsten oxide nanowires and the reduction of GO sheets to the formation of sandwichstructured tungsten oxide-reduced graphene oxide composite[14]. The intercalation of electrons and $\mathrm{Li}^{+}$into $\mathrm{W}_{18} \mathrm{O}_{49}$ to form lithium tungsten bronzes leading to the coloration process. Therefore, as discussed earlier intercalation and deintercalation of lithium ions from the electrolyte and electrons from the electrode play an important role in electrochromism. The strongly coupled effect between the tungsten oxide nanowires and RGO nanosheets exhibits high quality electrochromic performance with fast color switching speed, good cyclic stability and coloration efficiency. The assembled view of the electrochromic window is shown in Figure 1.2(a). The RGO 
sheets possess large surface area and small amount of residual oxygen-containing groups, which can favorably adsorb the $L i^{+}$ions via electrostatic interactions[25]. Also, the RGO sheets with low oxidation are electrical conducting and have a certain degree of carrier mobility[26]. Due to the covalent chemical bonding between the WONWs and the RGO sheets, a rapid, effective, and facile charge transport occurred during the whole electrochromic process, as schematically shown in Figure 1.2(b). Therefore, the coloration and bleaching processes could be completed in a short time. As shown in Figure 1.2(c), the structure of monoclinic $W_{18} O_{49}$ is constructed by pentagonal columns that are linked by an edge-sharing octahedron, leading to the occurrence of hexagonal, quadrangular and triangular tunnels [14]. These tunnels provide sufficient opportunity for the intercalation and de-intercalation of $\mathrm{Li}^{+}$ions. Importantly, the WONWS grown with the GO sheets as templates have huge aspect ratios (with diameter as thin as $1 \mathrm{~nm}$ and length up to few microns) and are welldispersed (no agglomeration happened), which could reduce the diffusion paths of $\mathrm{Li}^{+}$ions significantly. As a result, the WONWS-RGO composite exhibited not only faster $\mathrm{Li}^{+}$diffusion coefficient but also higher $\mathrm{CE}$ than the reported pure $W_{18} \mathrm{O}_{49}$ nanostructures [26].

\subsection{Organic ECWs}

In applications where lightness, thickness and lower power consumption are desired organic materials that can be precisely printed, sprayed, spin coated, stamped, drop casted into predefined patterns offer competitive alternative to inorganic counterparts. However, solution-processability, solid-state ordering, efcient electroluminescence, stable color-switching, and high charge-carrier mobility are difficult to match up with inorganic materials.

Many organic materials exhibit redox states with distinct electronic (UV/visible) absorption spectra. Where the switching of redox states generates new or different visible region bands, the material is said to be electrochromic[27]. Colour changes are commonly between a transparent ('bleached') state, where the chromophore only absorbs in the UV region, and a coloured state or between two coloured states. Where 


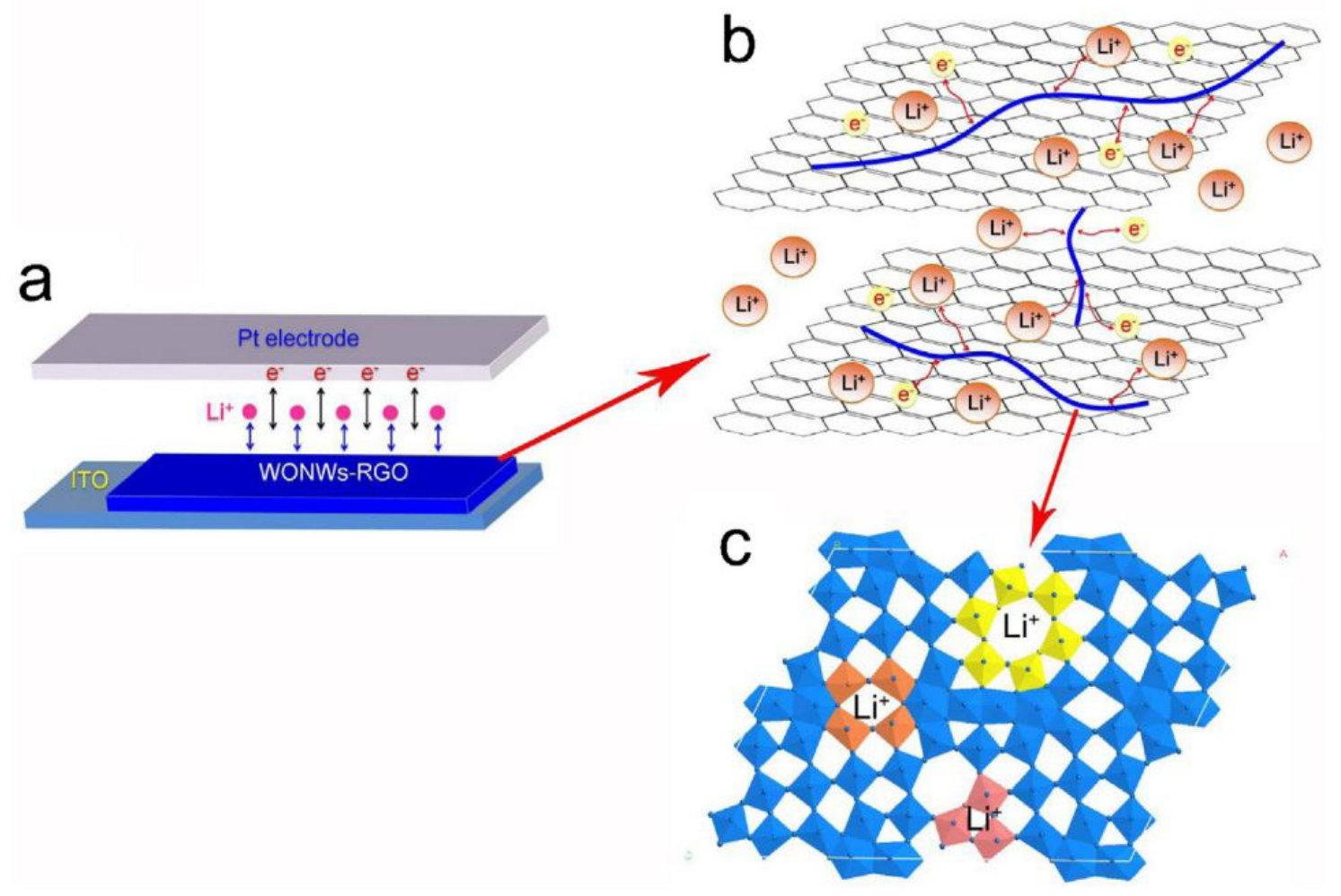

Figure 1.2: (a) Setup of WONWS-RGO composite-based EC system, (b) schematically structure illustrating the electrochromism of WONWS-RGO composite, and (c) crystal structure of monoclinic $W_{18} \mathrm{O}_{49}$ containing different tunnels that can accommodate $\mathrm{Li}^{+}$ ions $[14]$. 
more than two redox states are electrochemically accessible in a given electrolyte solution, the electrochromic material may exhibit several colours and be termed polyelectrochromic.The organic electrochromic windows are of three basic types, type 1 where electrochromic materials are soluble in both the reduced and oxidized state in a given electrolyte solution[28], for example, 1,1-di-methyl-4,4-bipyridilium(methyl viologen) disolves in both oxidized and reduced states, for such materials soluble electrochemically generated product material diffuses away and the current flow needs to be maintained until the whole solution becomes electrolysed to maintain its optical properties in a given state. Type II electrochromic materials where only one of the reduced or oxidized states is soluble, for example 1,1-di-heptyl-4,4-bipyridilium(heptyl viologen). In type III materials, such as conductive polymers, both redox states are solids and such systems are studied as solid thin films on substrates[27]. Type II and III materials have optical memory which means that once the redox state has been switched, no further charge injection is needed to retain the new electrochromic state $[27]$.

\subsubsection{Viologen systems}

Type (III) ECDs comprise viologen-modified working electrodes and counter electrodes made of inorganic redox film, such as Prussian blue or $\mathrm{V}_{2} \mathrm{O}_{5}$ film. The degree of color change, the color changing voltage and the charge capacities of the inorganic films do not match very well with organic viologen modified electrodes. This kind of low match leads to lower stability of type III device than type I or II [29]. In view of the above advantages and disadvantages, we focused on exploring improvement of stability for type III devices. Triphenylamine is one kind of organic anodic electrochromic material. Being electron-rich, triphenylamine is easily oxidized to form stable polarons and a noticeable change in color always accompanies the oxidation process [30]. For triphenylamine itself, it shows colorless in the neutral state and green in the oxidized state. While for its derivatives, the color depends on their structures. In addition, small organic electrochromic molecules of viologen and 
triphenylamine, the existing way have similarities intrinsically.

Viologens have become popular organic electrochromic material due to its wide availability and ease of varying the quaternizing agent. Viologen has three common redox states, shown in Figure 1.3, of which dication is the most stable and is colorless when pure unless optical charge transfer with counter anion occurs. Reductive electron transfer to the viologen dication forms the radical cation, the stability of which is attributable to the delocalisation of the radical electron throughout the framework of the bipyridyl nucleus, the 1 and 1 substituents commonly bearing some of the charge[31].
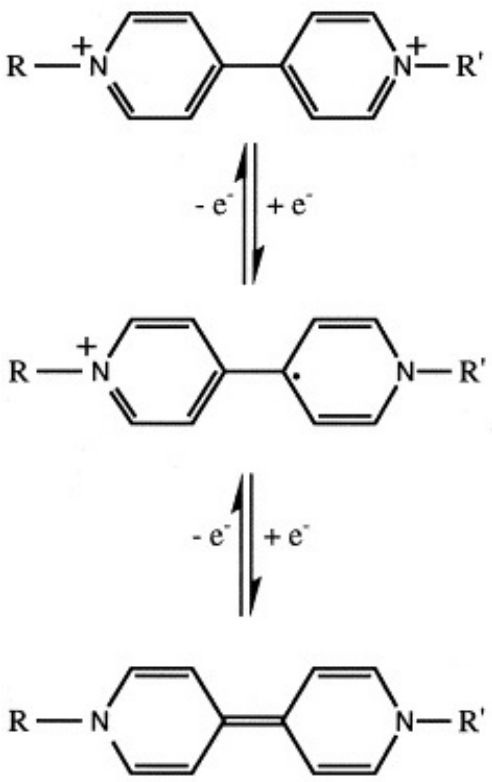

Figure 1.3: Three common redox states of viologen[31].

The viologen radical cations are intensely colored, with high molar absorption coefficients, owing to optical charge transfer between the +1 and zero valent nitrogens. Suitable choice of nitrogen substituents in viologens to attain the appropriate molecular orbital energy levels can, in principle, allow color choice of the radical cation. For example, alkyl groups promote a blue/violet color whereas the radical cation of 1,1-bis(4-cyanophenyl)-4,4-bipyridilium (cyanophenyl paraquat, CPQ) [32] 
in acetonitrile has an intense green color $(83,300 \mathrm{dm} 3 \mathrm{~mol} 1 \mathrm{~cm} 1$ at the $\max (674$ $\mathrm{nm})$ ). The intensity of the color exhibited by di-reduced viologens, Figure 1.3, is low since no optical charge transfer or internal transition corresponding to visible wavelengths is accessible.

One of the interesting electrochromic devices developed using viologen is the automatic dimming interior Night Vision Safety(NVS) mirror commercialized by Gentex. This system functions wholly by solution electrochromism, an ITO glass surface and reflective metallic surface, spaced a fraction of a millimeter apart from the two electrodes of the cell with a solvent containing two electroactive chemical species that function as electrochromic material and electrolyte. Electroactive species comprises of viologen, which is the cathodic coloring electrochromic species and a negatively charged phenylene diamine as the anodically coloring electrochromic material. After switching the device on, the species migrate to the respective electrodes. Once the dual electrochromic coloration process has begun, the products will diffuse away from their respective electrodes and meet in the intervening solution, where a mutual reaction regenerating the original uncolored species takes place. This type of electrochromic device therefore requires application of a continuous small current for replenishment of the colored electroactive species lost by their mutual redox reaction in solution. Bleaching occurs at short or open circuit by homogeneous electron transfer in the bulk of the solution. Although not an electrochromic phenomenon, the ingenious control system for this device is noteworthy. A photosensitive detector is placed facing rearward to monitor any dazzling incident light. However, this would also be triggered in daylight, resulting in an unwanted darkening of the mirror. This problem is avoided by a second forward-looking detector, which, on seeing daylight, is programed to cancel any operation of the controlling sensor, which therefore only responds at night[28]. 


\subsubsection{Conjugated electrochromic polymers}

Pi-conjugated organic polymers combining mechanical exibility, and ease in bandgap/color-tuning via structural control, along with the potential for low-cost scalability and processing are attractive as electrochromic materials. Chemical or electrochemical oxidation of numerous resonance-stabilized aromatic molecules, such as pyrrole, thiophene, aniline, furan, carbazole, azulene, indole, and others, produces electronically conducting polymers[33]. Polymerization reaction involves radicalcation/radical-cation coupling or reaction of a radical cation and a neutral monomer. The example of radical-cation/radical-cation coupling is illustrated in Figure 1.4 through the mechanism of electropolymerization of five-membered heterocycle, pyrrole.

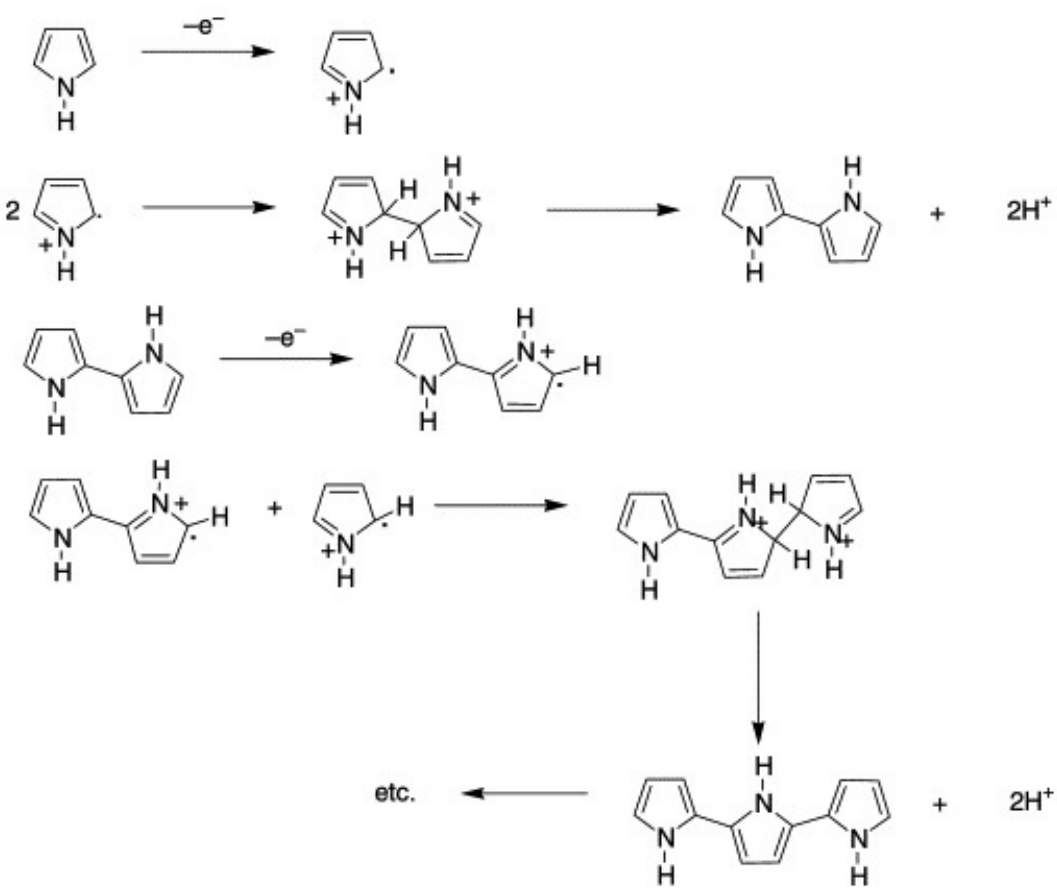

Figure 1.4: Proposed mechanism of electropolymerization of pyrrole. Example for radicalcation/radical-cation coupling[28].

During polymerization, after losing two protons and re-aromatization, the dimer 


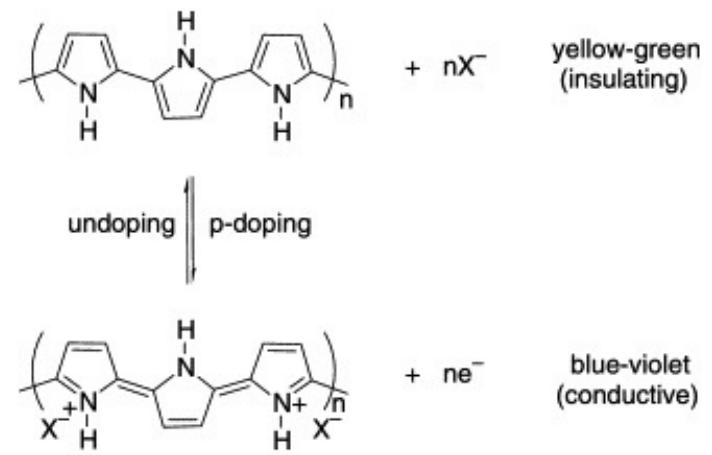

Figure 1.5: Polypyrole exhibiting electrochromism [34].

forms from the di-hydro dimer dication, The dimer and the succeeding oligomers can be more easily oxidized than the monomer and the resulting dimer radical cation undergoes further coupling reactions, proton loss and re-aromatization. Electropolymerization proceeds through successive electrochemical and chemical steps according to a general $\mathrm{E}(\mathrm{CE}) \mathrm{n}$ scheme, until the oligomers become insoluble in the electrolyte solution and precipitate onto the electrode surface[34]. In this manner, high-quality oxidized conducting films can be formed directly.

In the oxidized state, the conducting polymer film is charge balanced, doped with counter anions, also called p-doping and have a delocalized pi-electron band structure. Reduction of p-doped conducting polymer film by electrolyte cation incorporation removes the electronic conjugation to give neutral, undoped electrically insulating form. The band gap, $E_{g}$ between the highest occupied pi-electron band and lowest unoccupied pi-electron band determines the intrinsic properties of these materials. This can e illustrated through polypyrole, Figure 1.5 [34].

In some cases, the neutral state undergoes reductive cathodic doping with cation injection, n-doping to balance the injected charge. However, the stability of the negatively charged polymer state is limited and n-doping is harder to achieve. The similarity between conducting polymers and doped semiconductors arises from the manner in which the redox charges in the polymer change its optoelectronic properties. 
All conducting polymers are potentially electrochromic, redox switching giving rise to new optical absorption bands in accompaniment with simultaneous transport of electronic charge and counter ions in the polymer matrix. Oxidative p-doping shifts the optical absorption band towards the lower energy part of the spectrum. The color change or contrast between doped and undoped forms of the polymer depends on the magnitude of the bandgap of the undoped polymer. Thin films of conducting polymers with $E_{g}$ greater than $3 \mathrm{eV}(400 \mathrm{~nm})$ are colorless and transparent in the undoped form, while in the doped form they are generally absorbing in the visible region. Those with $E_{g}$ equal to or less than $1.5 \mathrm{eV}$ (800 nm) are highly absorbing in the undoped form but, after doping, the free carrier absorption is relatively weak in the visible region as it is transferred to the near infrared. Polymers with intermediate gaps have distinct optical changes throughout the visible region and can be made to induce many color changes[28].

ProDOT-Me2 as electrochromic material: Polythiophenes are of interest as electrochromic materials due to their ease of chemical and electrochemical synthesis, environmental stability, and processability. A large number of substituted thiophenes has been syn- thesized, and this has led to the study of numerous novel polythiophene(s), with particular emphasis on poly(3- substituted thiophenes) and poly(3,4disubstituted thiophenes) [33]. Thin polymeric films of the parent polythiophene are blue $(730 \mathrm{~nm})$ in the doped (oxidized) state and red $(470 \mathrm{~nm})$ in the undoped form. However, due to its lower oxidation potential, the electropolymerization and switching of 3-methylthiophene have been more intensively studied than the parent thiophene. Furthermore, the introduction of a methyl group at the b position of the thiophene ring leads to a significant increase of the polymer conjugation length and hence electronic conductivity [33]. This effect has been attributed to the statistical decrease in a number of a-b0 couplings and to the decrease of the oxidation potential caused by the inductive effect of the methyl group [33]. Poly(3-methylthiophene) is purple when neutral with an absorption maximum at $530 \mathrm{~nm}(2.34 \mathrm{eV})$ and turns pale blue on oxidation[35].

The evolution of the electronic band structure during electrochemical p-doping of 


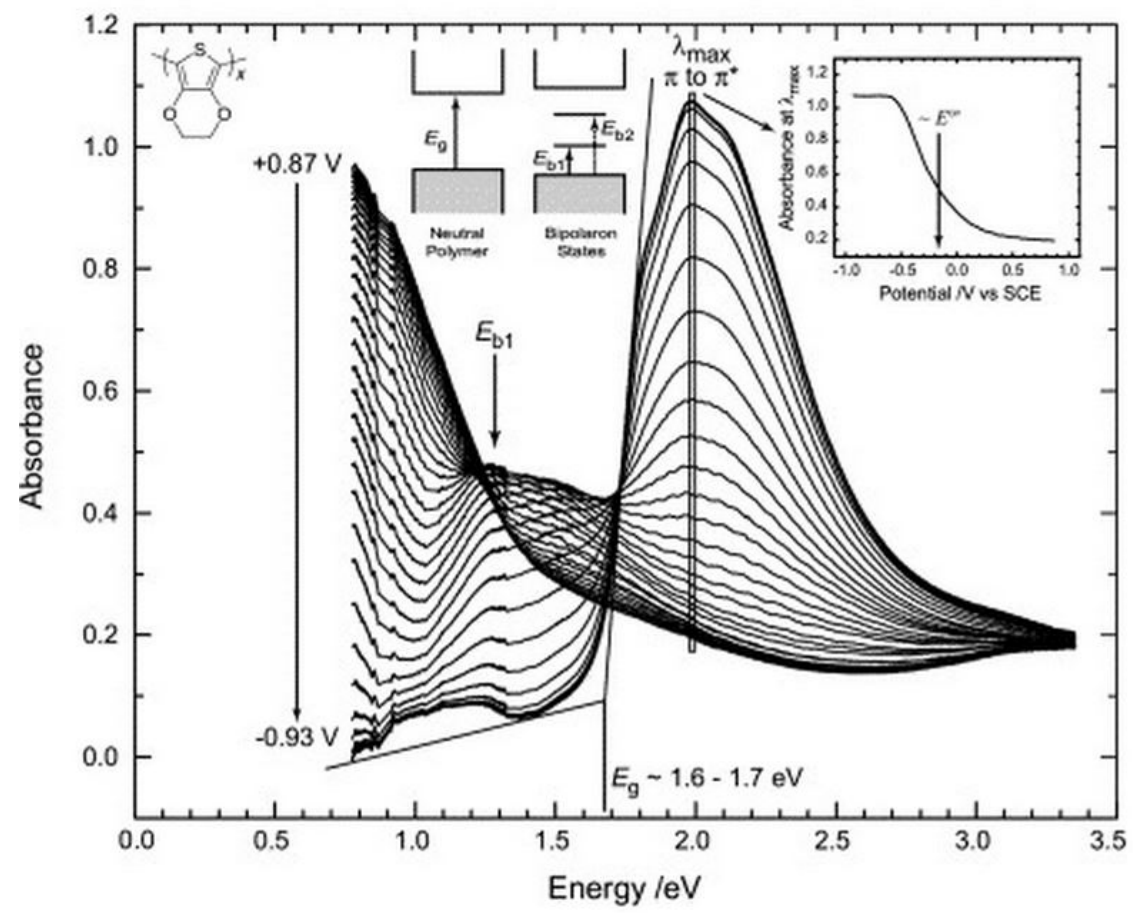

Figure 1.6: Spectroelectrochemistry for PEDOT film on ITO[36].

electrochromic polymers can be followed by recording in situ visible and NIR spectra. shown in Figure 1.6 shows thespectroelectrochemical series for an alkylenedioxysubstituted thiophene polymer, poly(3,4-(ethylenedioxy)thiophene). The undoped polymer's strong absorption band, with a maximum at $621 \mathrm{~nm}(2.0 \mathrm{eV})$, is characteristic of a interband transition. Upon doping, the interband transition decreases, and two new optical transitions (at 1.25 and $0.80 \mathrm{eV}$ ) appear at lower energy, corresponding to the presence of a polaronic charge carrier (a single charge of spin 1/2). Further oxidation leads to formation of a bipolaron and the absorption is enhanced at lower energies. The characteristic absorption pattern of the free carrier of the metallic-like state then appears when the bipolaron bands finally merge with the valence and conduction bands.

Tuning of color states is possible by suitable choice of thiophene monomer. This represents a major advantage of using conducting polymers for electrochromic ap- 
plications. Subtle modifications to the monomer can significantly alter spectral properties. For example, the colors available with polymer films prepared from 3methylthiophene-based oligomers are strongly dependent on the relative positions of methyl groups on the polymer backbone [36]. Colors available include pale blue, blue and violet in the oxidized form, and purple, yellow, red and orange in the reduced form. The color variations have been ascribed to changes in the effective conjugation length of the polymer chain. Cast films of chemically polymerized thiophene-3-acetic acid reversibly switch from red to black on oxidation, demonstrating that subtle changes in structure can cause large effects in the colored states.

Study of the effects of steric factors is provided by the electronic properties of polythiophenes with 3,4-dialkyl substituents. In principle, disubstitution at the, positions should provide the synthetic basis to perfectly stereoregular polymers. However, this approach is severely limited by the steric interactions between substituents, which lead to a decrease in polymer conjugation length. In fact, poly(3,4-dialkylthiophenes) have higher oxidation potentials, higher optical bandgaps, and lower conductivities than poly(3-alkylthiophenes) [34]. Cyclization between the 3 and 4 positions relieves steric hindrance in thiophenes, but many are harder to electropolymerize than, say, 3-methylthiophene.

In 1999, dipropoxythiophene with dimethyl substituted at , positions,Dimethyl 3,4-dipropoxythiophene(ProDOT-Me2) was first synthesized [34]. The methyl group at , positions does not give rise to significant steric interactions. In the publication the synthesis of ProDOT was performed through williamson ether synthesis with 1,3 dibromopropane and 3,4-dihydroxythiophene-2,5diethylcarboxylate and subsequent ester hydrolysis and decarboxylation, shown in Figure 1.7.

In this case, higher dialkoxythiophene like 3,4-diethoxy and 3,4dipropoxythiophene which exhibits enhanced storage stability can also function as starting materials. The di-hydroxy functional compound is generally observed as the by product especially when excessive neopentyl glycol is used to ensure that the reaction is complete.

Reynolds et. al have reported several intriguing for electrochemically prepared 


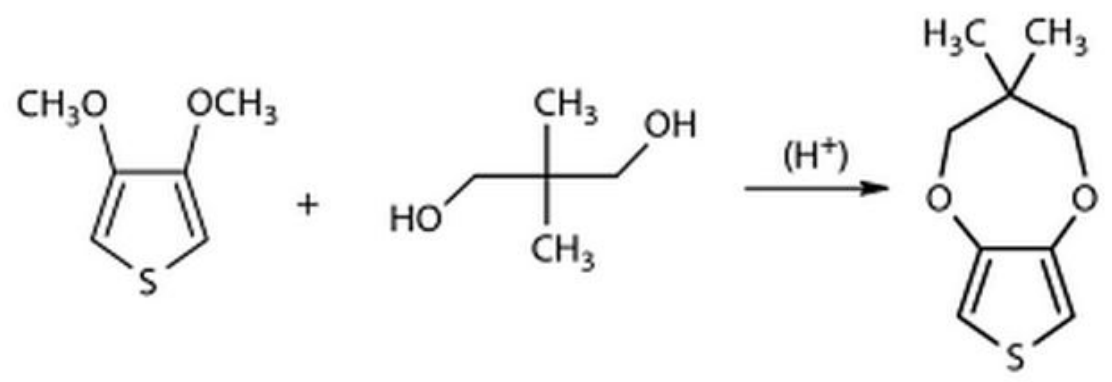

Figure 1.7: Synthesis of ProdDOT-Me 2 [34]

ProdDOT-Me2 when depoisted as a film on a conducting surface. The switching speed of ProdDOT-Me2 from almost fully transparent, oxidized state to dark blue, reduced state is an order magnitude higher thanits polyalkylthiophene counterparts and about three to seven times higher than PEDOT. Compared to poly(3,4-ethylene dioxythiophene):poly(styrene sulfonate) (PEDOT:PSS), electropolymerized PEDOT or other derviatives of ProDOT, transmittance change between the doped and undopped state is significantly higher in ProDOT-Me 2 .

\subsection{Organometallic ECW}

Metallo-supramolecular materials, also called coordination polymers were recently introduced as a new field for electrochromic applications. Researchers combine the inorganic metal ions with organic ligands to form inorganic-organic hybrid systems. Because the interactions between metal centers and ligands influence the characteristics of coordination polymers directly, redox-active transition metal coordination complex exhibit intense color changes due to metal-to-ligand charge transfer (MLCT) [36]. At increasing applied potentials the metal centers are oxidized to higher valence state and the MLCT decreases. Optically this results in a decrease in the visible absorbance caused by the coordinated polymer switching from the colored to the transparent state [37]. Therefore, both the selection of metal ion centers and ligands play an important role on electrochromic characteristics in coordination polymer system 
and influence the performance in electrochemical and optical properties[38].

Among the metallo-supramolecular materials, metallo-supramolecular coordination polyelectrolytes (MEPE) series belong to coordination polymers having homotopic bis-terpyridine ligand and transition metal ions [39], shown in figure 8. 2,2 - 6,2-terpyridines have high binding affinity with transition metal ions to form a well-defined octahedral structure [40]. Due to dp back bonding of the metal to the pyridine rings and the chelate effects, high binding affinity offers the thermodynamic driving force for metallo-supramolecular formation [41]. Han et al. have investigated a series of MEPEs as electrochromic materials and the research focuses on pyridine-ring functionalized bisterpyridines linked with rigid, linear phenyl spacers with different transition metal ions (Fe(II), $\mathrm{Ru}(\mathrm{II})$ and $\mathrm{Co}(\mathrm{II})$ )[42]. The function of substituents on the terpyridines is to influence the strength of coordinate bond and MLCT changes with difference between maximal absorbance wavelength, electrochemical and electrochromic properties [43]. Their research resulted in a heterometallic MEPE consisting of equivalent Fe(II) and $\mathrm{Co}(\mathrm{II})$. Through mixing MEPE-Fe(II) and MEPE-Co(II), the polymer blend exhibits two steps of color changes (Purple $\rightarrow$ blue $\rightarrow$ transparent).

Chen et al developed a new cathodically coloring electrochromic material based on metallo-supramolecular polyelectrolyte containing $\mathrm{Cu}(\mathrm{I})$ metal ion $(\mathrm{MEPE}-\mathrm{Cu}(\mathrm{I})$ ). The electrochromic mechanism for MEPE series involves the redox reaction of $\mathrm{Cu}(\mathrm{I}) / \mathrm{Cu}(\mathrm{II})$ pair and MLCT. Both the redox states would drive MEPEs to change color upon the passage of a small reaction charge. The observed transmittance change of MEPC$\mathrm{Cu}(\mathrm{I})$ thin film at $580 \mathrm{~nm}$ is approximately $20 \%$ with applied potential between 0 to $1.5 \mathrm{~V}$. It has a fast response time of less than 1s and a coloration efficiency value of 260 $\mathrm{cm}^{2} / \mathrm{C}$ at $580 \mathrm{~nm}$. The electrochromic device was fabricated by choosing polyanilinecarbon nanotube to act as the ion storage layer for MEPE-Cu(I). A liquid electrolyte containing tetrabutylammonium perchlorate in acetonitrile with perchloric acid is sandwiched between the working and the counter electrode [43]. 


\section{Chapter 2}

\section{APPLICATIONS OF ELECTROCHROMIC WINDOWS}

Electrochromic windows have evolved to be the most promising switchable window technology today. The electrochromic window contains thin film stack that is deposited on a glass substrate. The stack consists of conductive oxide layer, electrochromic material, ion storage material and electrolyte. When a voltage is applied between the transparent conducting oxide, a distributed electrical field is set up. This field moves Lithium ions between the ion storage film through the electrolyte into the electrochromic film. The effect of this movement is that the window switches between transparent and opaque state.

Low voltage power requirement is one of main advantages of using ECW. The ECW can be modulated to intermediate states between clear and fully colored state. In the tinted state solar radiation is absorbed and becomes dark. Whereas in the transparent state most of the solar radiation passes through the window making it transparent. Lower emittance coatings and an insulating glass unit configurations or thermochromic layer coating can be used to reduce heat transfer from this absorptive glazing layer to the interior. Typical ECWs have a visible transmittance between 0.50 to 0.70 and a lower range of 0.02 to 0.2544 . a low transmission is desirable for privacy during the day and for control of direct sunlight and glare, potentially eliminating the need for interior shading. A high transmission is desirable for admitting daylight during the time of the day that the sun is not shining directly into the space, during overcast periods and it can also be used for passive solar heating in winter. Therefore, greater the range of transmission, the more able the window is to satisfy a wide range of environmental requirements.

Generally in polymeric ECWs when the device is switched to the desired state and maintains the desired state without any additional power supply. Inorganic ECWs 
have color memory making them more energy efficient. Other type of ECWs require minimal low-voltage power to both change and maintain the desired transmittance level. Inorganic ECWs have proven to be very stable under hot and cold conditions and under intense sun. The commercially available ECWs are expected to have a lifetime between 20 to 30 years.

The switching speed of ECW is defined as the time taken by the ECW to switch $90 \%$ of its color. Switching speed is closely related to the size and operation temperature of the window. Typically in ECW switching from colored to transparent state takes longer than switching from transparent to colored state. Organic ECWs have better switching speed compared to their inorganic counterparts. In buildings a gradual transmission change is advantageous since it allows the occupants eyes to get adjusted to the change in light intensity entering the room without causing discomfort. Modulating the amount of energy entering the room is a technique widely used by lighting designers to prevent occupant distraction.

In applications like sunroofs in automobiles organic ECWs would be more suitable since they have faster switching speed compared to their inorganic counterparts. In automobile applications when the driver wishes to change the transmittance of the sunroof it should respond immediately for safety purposes. The driver can also choose intermediate level of transmittance for enhanced comfort.

Controlling and modulating the light and amount of heat entering the room leads to lower energy bills and increased comfort. ECWs are initially expensive but the cost can be partially offset by lower electricity bill. In the long run, the cost of installing ECW is cheaper than installing static glass and additional HVAC equipments. Electrochromic window give building owners the ability to modulate heat gain through the window, reducing cycling stress on HVAC motors and other equipment.

\subsection{Electrochromic windows as sunroof in automobiles}

As discussed earlier, organic ECWs would best suit for application as sunroof in automobiles. In order to use organic ECW in automobiles the window should be capable of operating under harsh environment. In Figure 2.1 it can be observed 


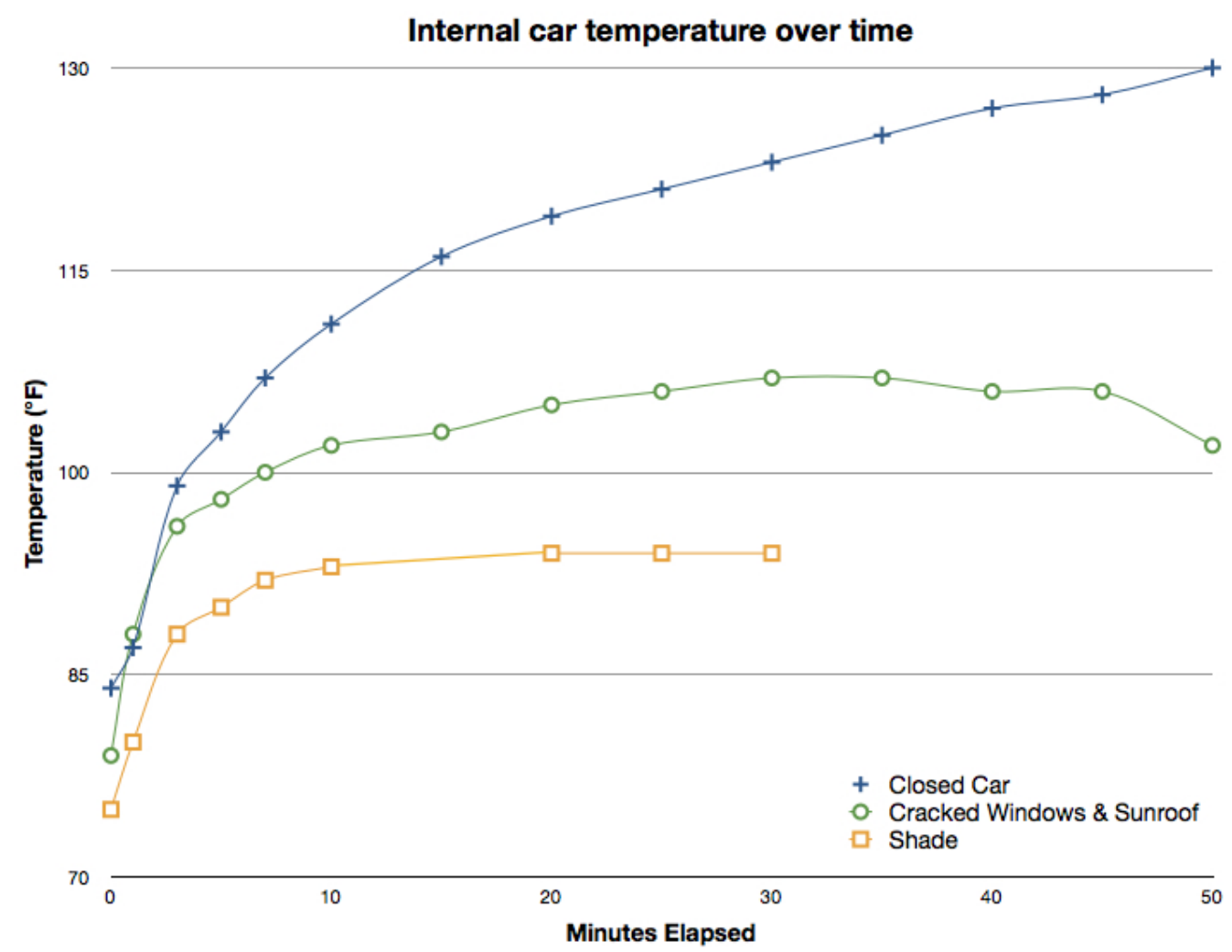

Figure 2.1: Heating curves for the air inside a parked car under different conditions [45]

that when the automobile is parked directly under the sun or shade it experiences a significant increase in temperature. When the automobile is parked under the sun the temperature on sunroof can reach upto $90^{\circ} \mathrm{C}$ and when the automobile is parked directly under the sun the temperature may rise upto $130^{\circ} \mathrm{C}$.

The rise in internal car temperature is due to greenhouse effect. The greenhouse effect refers to circumstances where the short wavelengths of visible light from the sun pass through a transparent medium and are absorbed, but the longer wavelengths of the infrared re-radiation from the heated objects are unable to pass through that medium. The trapping of the long wavelength radiation leads to more heating and a higher resultant temperature. Besides the heating of an automobile by sunlight 


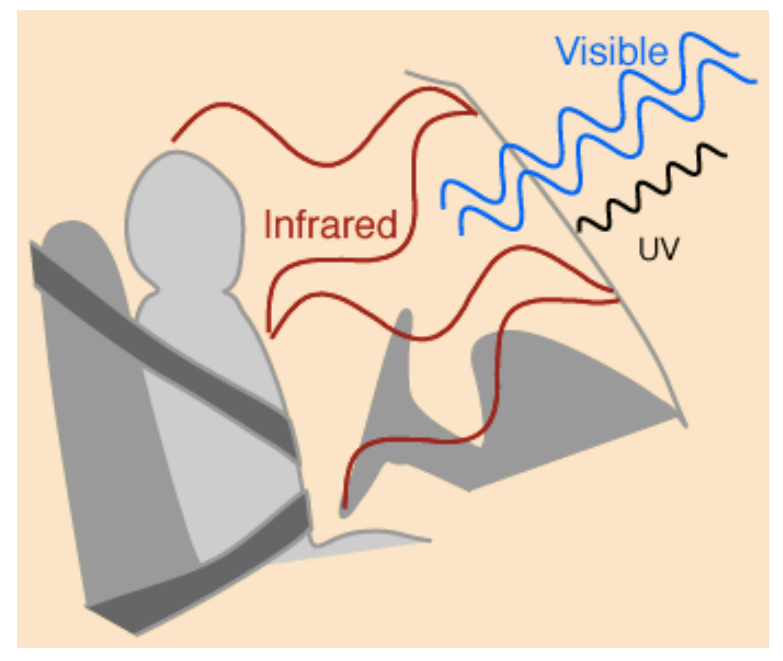

Figure 2.2: Short wavelengths of visible light are readily transmitted through thetransparentwindshield. [46]

through the windshield and the example of heating the greenhouse by sunlight passing through sealed, transparent windows, the greenhouse effect has been widely used to describe the trapping of excess heat by the rising concentration of carbon dioxide in the atmosphere. The carbon dioxide strongly absorbs infrared and does not allow as much of it to escape into space.

Bright sunlight will effectively warm the car on a cold, clear day by the greenhouse effect. The longer infrared wavelengths radiated by sun-warmed objects do not pass readily through the glass. The entrapment of this energy warms the interior of the vehicle. The trapping of the hot air so that it cannot rise and lose the energy by convection also plays a major role. Shorter wavelengths of ultraviolet light are largely blocked by glass since they have greater quantum energies which have absorption mechanisms in the glass [46].

\subsubsection{Problem Statement}

The aim of this study is to develop ECWs that can be used as sunroofs in automobiles. As discussed earlier, the sunroofs in automobiles experience high temperature 
environment throughout the day. Therefore, it is important to design an ECW that can function efficiently in a wide range of temperature. In this study, an ECW is developed to operate with good optical contrast and switching speed in the temperature range of $20^{\circ} \mathrm{C}$ to $120^{\circ} \mathrm{C}$. The individual components of ECW are tested for heat resistance. The gel electrolyte was found to be the most vulnerable component of ECW and a new gel electrolyte with higher heat resistance has been developed.

\subsubsection{Components of $E C W$}

ECW primarily contains three main components,

1. Working electrode(WE) which is generally chosen as the electrochromic material,

2. electrically conductive counter electrode (CE) that acts as ion storage material which stores Lithium cations and releases Lithium ions into the electrolyte. This gives stability to the ECW,

3. a transparent electrolyte.

The electrochromic material and the counter electrode materials are deposited on a conductive oxide like Indium Tin Oxide(ITO) coated glass substrate. The conductive oxide coated glass substrate gives the device more stability and improves their electrical conductivity. Depending on the electrochromic material the material can become transparent when oxidized and colored when neutral. In this study, poly $(3,3-$ dimethyl-3,4-dihydro-2H-thieno[3,4-b][1,4]dioxepine), PPRODOT-Me $\mathrm{Me}_{2}$ as a working electrode, $\mathrm{V}_{2} \mathrm{O}_{5}-\mathrm{TiO}_{2}$ composite materials as a counter electrode and poly(ethylene imine) based electrolyte was used to develop heat resistant electrochromic window.

Once assembled the transmittance of the ECW can be tuned by varying the applied voltage. When positive voltage is applied to the working electrode electrons are drawn from the electrochromic film thus oxidizing the film. The oxidized PPRODOT- $\mathrm{Me}_{2}$ is highly transparent and has a transparency of $71 \%$ at $580 \mathrm{~nm}$ in room temperature, shown in Figure 2.3. 


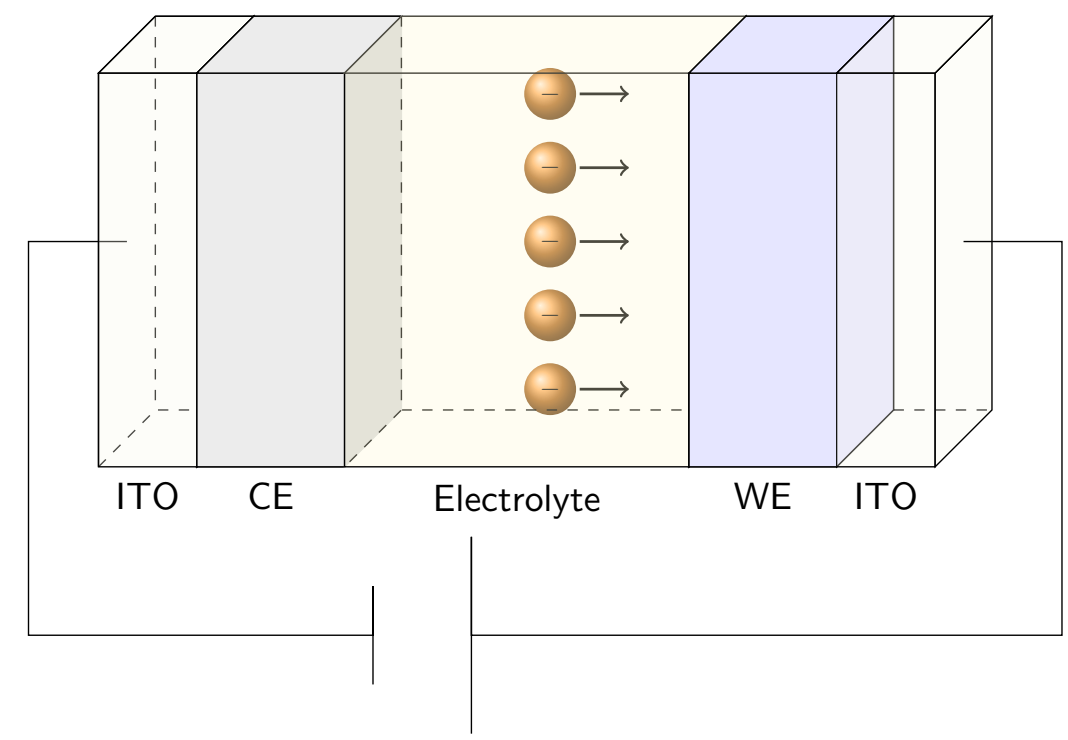

Figure 2.3: Working of ECW, positive voltage applied PProDOT-Me2 film. The film is oxidized and turns light blue with high transmittance of $71 \%$.

On the other hand, when negative voltage is applied to the working electrode the electrons are inserted into the PPRODOT-Me $\mathrm{M}_{2}$ thus making the film neutral, shown in Figure 2.4. The neutral PPRODOT-Me $\mathrm{Me}_{2}$ turns dark blue and opaque with a transmittance less than $5 \%$. When negative voltage is applied to the working electrode the Lithium cations are attracted to it. The insertion of Lithium ions into the PPRODOT-Me $\mathrm{M}_{2}$ film gives the film optical color memory. Thus the developed ECW is more energy efficient since it does not require continuous supply of voltage.

When negative voltage is applied to the counter electrode, shown in Figure 2.3, the film is reduced. The $\mathrm{V}_{2} \mathrm{O}_{5}-\mathrm{TiO}_{2}$ film turns grey upon reduction, the reduced state maintains high transmittance of over $75 \%$. Upon application of positive voltage, shown in Figure 11, the $\mathrm{V}_{2} \mathrm{O}_{5}-\mathrm{TiO}_{2}$ film is oxidized and turns light yellow. The oxidized state of the $\mathrm{V}_{2} \mathrm{O}_{5}-\mathrm{TiO}_{2}$ is highly transparent with a transmittance of around $80 \%$. Since the counter electrode material is highly transparent in the reduced state it does not reduce the performance of the ECW when operating in the transparent 


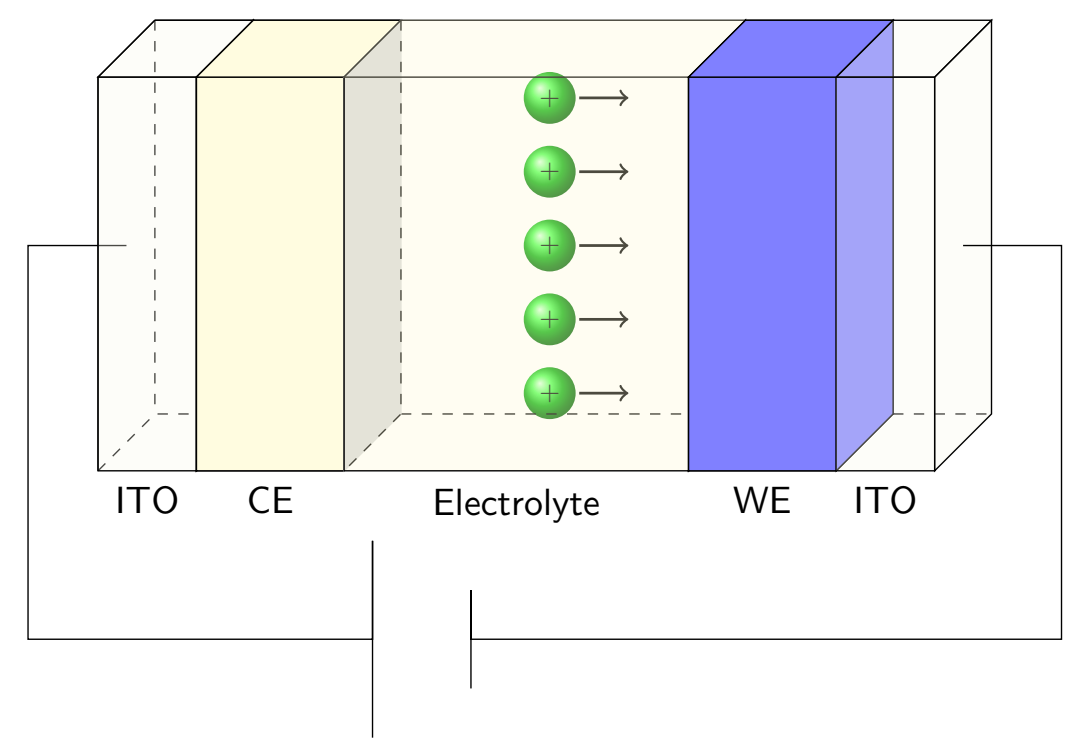

Figure 2.4: Working of ECW, negative voltage applied PProDOT-Me2 film. The film is reduced and turns dark blue with transmittance less than $5 \%$.

mode. Whereas, when the ECW is operating in the opaque mode, the electrochromic layer is dark blue while the ion storage material is light yellow which reduces the performance of the ECW. Ideally, the ion storage layer should have low transmittance in oxidized state to improve the performance of the ECW.

In this study, polyethylene imine based electrolyte was developed. The electrolyte used in the ECWs have three important requirements,

1. Should be highly transparent to not interfere with the working of the ECW,

2. Should have high ionic conductivity to ensure high switching speed between the opaque and transparent states in ECW,

3. the developed electrolyte should be compatible with the working and counter electrode material to ensure smooth functioning of ECW.

\section{PProDOT-Me $\mathrm{M}_{2}$ film as the working electrode}




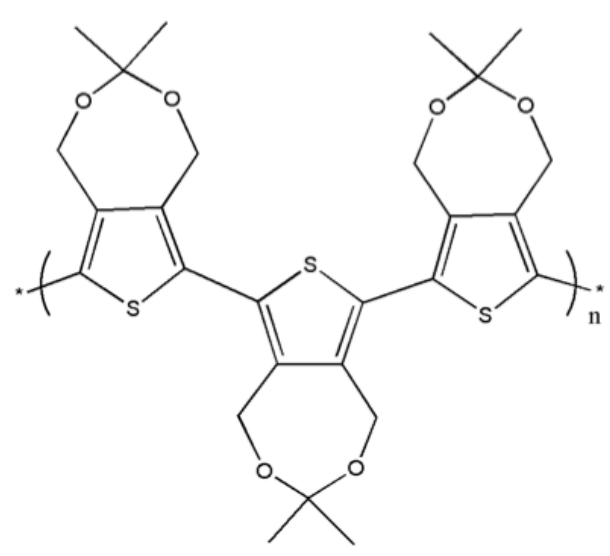

a)

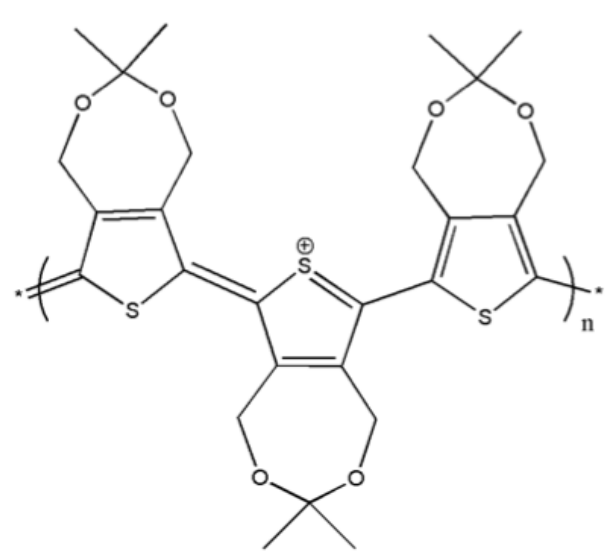

b)

Figure 2.5: ProDOT- $\mathrm{Me}_{2}$ structure in a) neutral and b) oxidized states[?]

The PProDOT-Me $-\mathrm{Me}_{2}$ film turns transparent upon oxidation and turns opaque upon reduction. This modification in the optical property of the conjugated polymer can be attributed to the modification to the structure of PProDOT-Me $\mathrm{Me}_{2}$ upon reduction and oxidation, shown in Figure 2.5.

When the conjugated polymer is infused with ions it becomes a conducting polymer. Increase in electron delocalization along the backbone of the polymer the conjugation length of the polymer increases which reduces the band gap between the highest occupied molecular level (HOMO) and lowest unoccupied molecular level (LUMO) level of the polymer. Creation of new band gap modulates the optical property of the conjugated polymer. This can be illustrated through Figure 2.6, as the conjugation length increases from ethylene to polyacetylene the band gap reduces. Therefore the energy required for an electron to jump from HOMO level to LUMO level reduces as the conjugation length increases.

The band gap affects the light absorbed by the conducting polymer by equation 2.1

$$
E=\frac{h c}{\lambda}
$$




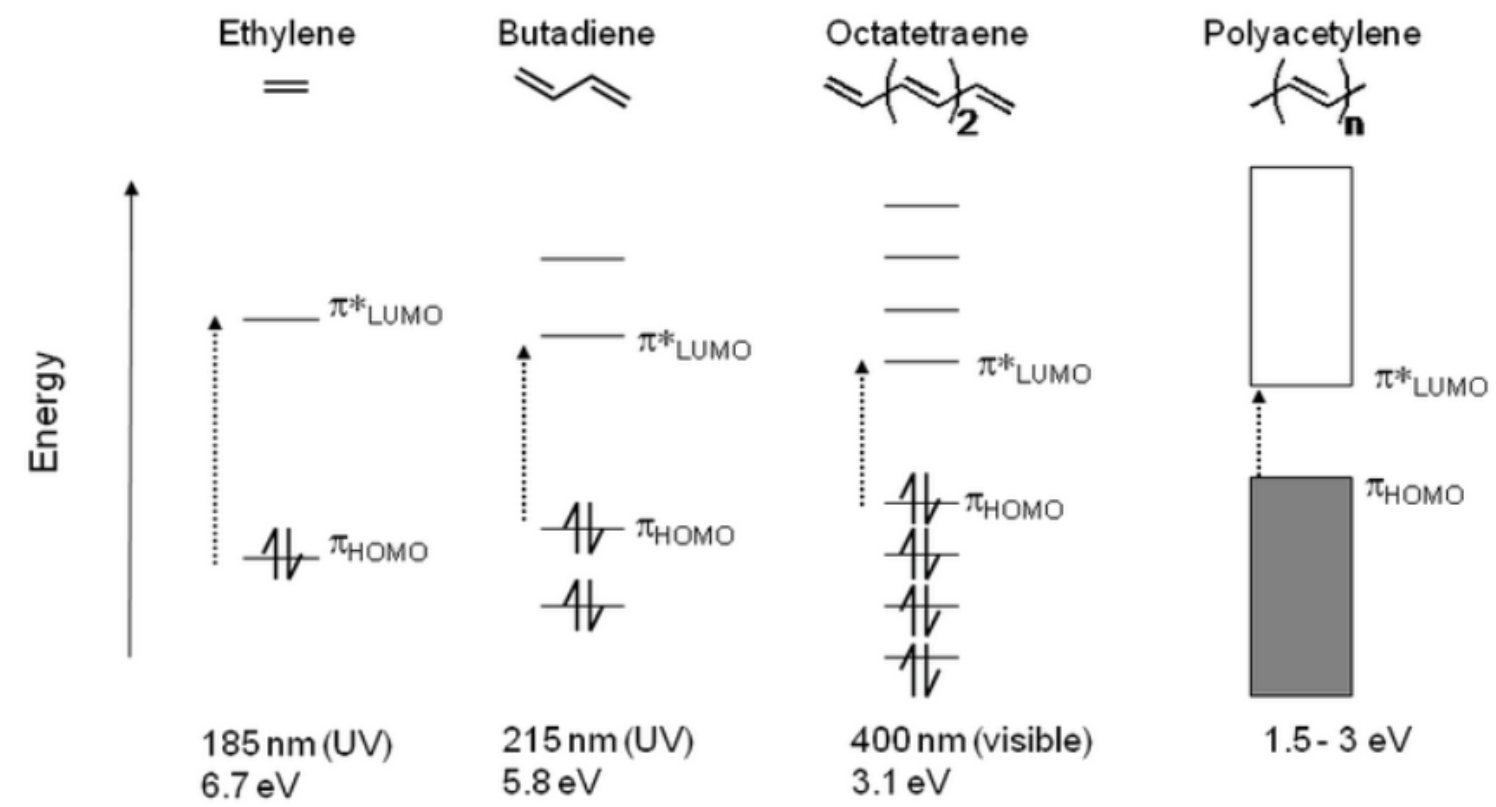

Figure 2.6: As the conjugation length increases from ethylene to polyacetylene the band gap decreases.

where $h$ is plancks constant, $c$ is the velocity of the light, $E$ refers to the band gap and $\lambda$ is the absorption wavelength. The $\pi-\pi^{*}$ transition is depleted at the expense of transition outside the visible region therefore the dominant wavelength of the color is the same throughout the doping process[49].

In PProDOT- $\mathrm{Me}_{2}$ the band gap is $1.7 \mathrm{eV}$ which corresponds to a wavelength of $715 \mathrm{~nm}$. By altering the wavelength we can alter the color of the conducting polymer.

\section{Electropolymerization of ProDOT-Me $\mathrm{Me}_{2}$}

An ITO coated glass $(6 \Omega /$ square, $76 \mathrm{~mm} \times 76 \mathrm{~mm}$ dimensions, Thin Film Devices) was washed with an ethanolamine aqueous solutions and rinsed with DI water. It was cleaned under UV ozone and dried at $110{ }^{\circ} \mathrm{C}$ overnight before use. A cleaned ITO/glass substrate, a platinum plate counter electrode and a silver wire reference electrode were submerged in a ProDOT- $\mathrm{Me}_{2} / \mathrm{LiClO}_{4} /$ acetonitrile $(\mathrm{ACN})$ solution. The PProDOT- $\mathrm{Me}_{2}$ coating was deposited by a chronoamperometry program in an 
electrochemical analyzer (CHI 605A, CH Instruments). A three electrode cell with $\mathrm{Ag} / \mathrm{Ag}^{+}$as a reference, ITO glass as a working electrode and a Pt plate $(25 \mathrm{~mm} \times$ $25 \mathrm{~mm} \times 1.0 \mathrm{~mm})$ as a counter electrode was used for electropolymerization. The overall experimental process was carried out at $25^{\circ} \mathrm{C}$ in argon atmosphere. The applied potential was $1.6 \mathrm{~V}$ and polymerization time varied from 2 to $15 \mathrm{~s}$. Monomer and electrolyte concentrations varied keeping one of them constant to evaluate their respective reaction orders. The electrolyte concentrations varied from $1.5 \times 10^{1}$ to $5 \times 10^{2} \mathrm{M}$ and the constant monomer concentration was $1 \times 10^{2} \mathrm{M}$. Monomer concentrations ranged from $2 \times 10^{2}$ to $5 \times 10^{3} \mathrm{M}$ with the constant electrolyte concentration, $1 \times 10^{1} \mathrm{M} . \mathrm{LiClO}_{4}$ in propylene carbonate $(\mathrm{PC})$ was used as the electrolyte for redox systems of the films. The total charge of the film was monitored as a function of time in a chronocoulometry program. Optical transmittance spectra of the films were examined in the wavelength range of $300800 \mathrm{~nm}$ using a UV/VIS/NIR spectrophotometer (V-570, JASCO, and MDT)[52].

ProDOT- $-\mathrm{Me}_{2}$ monomer should be polymerized on to the conducting oxide layer coated glass substrate to develop the working electrode for ECW. Two methods are generally employed to form conducting polymer films, they are

1. Chemical polymerization[50],

2. Electrochemical polymerization[51].

Chemical polymerization uses chemical oxidants resulting in the formation of more bulk like material precipitated from the reaction mixture. It involves immersion of solid substrate into the solution containing corresponding monomer and oxidant. Due to the monomer oxidation, the substrate is coated with thin polymer film. In this method, it is hard to control the thickness of the polymer film on the substrate. Whereas, electrochemical polymerization involves electrochemical oxidation of a corresponding monomer on a substrate that acts as an electrode, followed by precipitation of the polymer on the surface. In this method we can precisely control the thickness of the film. Therefore, electrochemical fabrication technique was 
used to develop PProDOT-Me $\mathrm{Me}_{2}$ film. Chronoamperometric and gravimetric techniques help us determine the general kinetic electropolymerization, the order of the reaction with respect to the monomer, solvent and the electrolyte. Generally, the electropolymerization reaction involves monomer dissolved in a suitable solvent. In the electrochemical cell, the monomer solution acts as the electrolyte and the polymerization reaction occurs at the surface of one of the electrodes. Therefore, the reaction can be summarized as,

$$
\text { electrolyte }(E)+\text { monomer }(M) \rightarrow \operatorname{doped}(\operatorname{polymer})(P)
$$

Therefore rate of polymerization, $R$, can be given by,

$$
R=W=k[M]^{m}[E]^{n}
$$

Where $[M]$ and $[E]$ refer to the concentrations of the monomer and the electrolyte respectively. The order of the reaction with respect to the monomer is $m$ and with respect to the electrolyte is $n$. With reaction rate of $R, W$ weight of polymer is deposited per square centimeter of electrode surface[52].

Kim et al calculated the thickness of the electropolymerized PProDOT- $-\mathrm{Me}_{2}$ film through chronoamperometry technique[52]. In the experiment, the polymerization charge density $\left(Q_{p}\right)$ with different electrolyte and monomer concentration. If the polymerized monomer is the only species produced then charge density would be proportional to the weight $(W)$ of the polymer produced[52],

$$
W=k^{\prime} Q_{p}
$$

Rate of polymerization can now be rewritten as,

$$
R=\frac{d Q}{d t}=\frac{1}{k^{\prime}} * \frac{d W}{d t}
$$

Now substituting the value of $W$ from the equation we get the rate in terms of the concentration of the monomer and the electrolyte. The rate of the reaction can be obtained through chronoapmerometry technique by plotting a graph of charge density versus polymerization time[52]. The orders of the reaction with respect 
to the monomer and electrolyte can be obtained from the logarithmic plot of rate of polymerization reaction versus log of concentration of monomer and electrolyte respectively.

To estimate the thickness of the PProDOT-Me $\mathrm{M}_{2}$ film,

$$
\begin{gathered}
W=\text { density } * \text { volume } \\
W=\operatorname{density} *(\text { area } * \text { thickness })
\end{gathered}
$$

$W=($ amount deposited of PProDOT-Me 2 film deposited/unit area/unit time $)$

$*$ Polymerization time $*$ area

Therefore,

Thickness of the film $=\left(\right.$ amount deposited of PProDOT-Me $\mathrm{M}_{2}$ film deposited $/$ unit area/unit time $)$ /density $*$ polymerization time.

Therefore, polymerization time plays a key role in determining the thickness of the film if other parameters are kept constant [52].

The optimized polymerization time to develop heat resistant ECW is 15 seconds. The charge capacity of the developed PProDOT-Me 2 film should match the charge capacity of the counter electrode film [52].

\section{Heat resistance of the PProDOT-Me $\mathrm{Me}_{2}$ film}

To develop a high temperature resistant ECW the individual components should be heat resistant. The heat resistance of PProDOT- $-\mathrm{Me}_{2}$ film was tested by subjecting the neutral and oxidized films at high temperature. The transmittance was measured at $20{ }^{\circ} \mathrm{C}, 40{ }^{\circ} \mathrm{C}, 60{ }^{\circ} \mathrm{C}, 80{ }^{\circ} \mathrm{C}, 100{ }^{\circ} \mathrm{C}, 120^{\circ} \mathrm{C}$ to measure the progressive variation in transmittance of the film over temperature, shown in Figure 2.7.

From Figure 2.7, we can observe that the contrast between the opaque and transparent states decreases at higher temperatures. The reduction in contrast is due to the decrease in transmittance in the oxidized state and increase in transmittance in the reduced state. The transmittance decreases by about $5 \%$ from $20{ }^{\circ} \mathrm{C}$ to $120{ }^{\circ} \mathrm{C}$ 


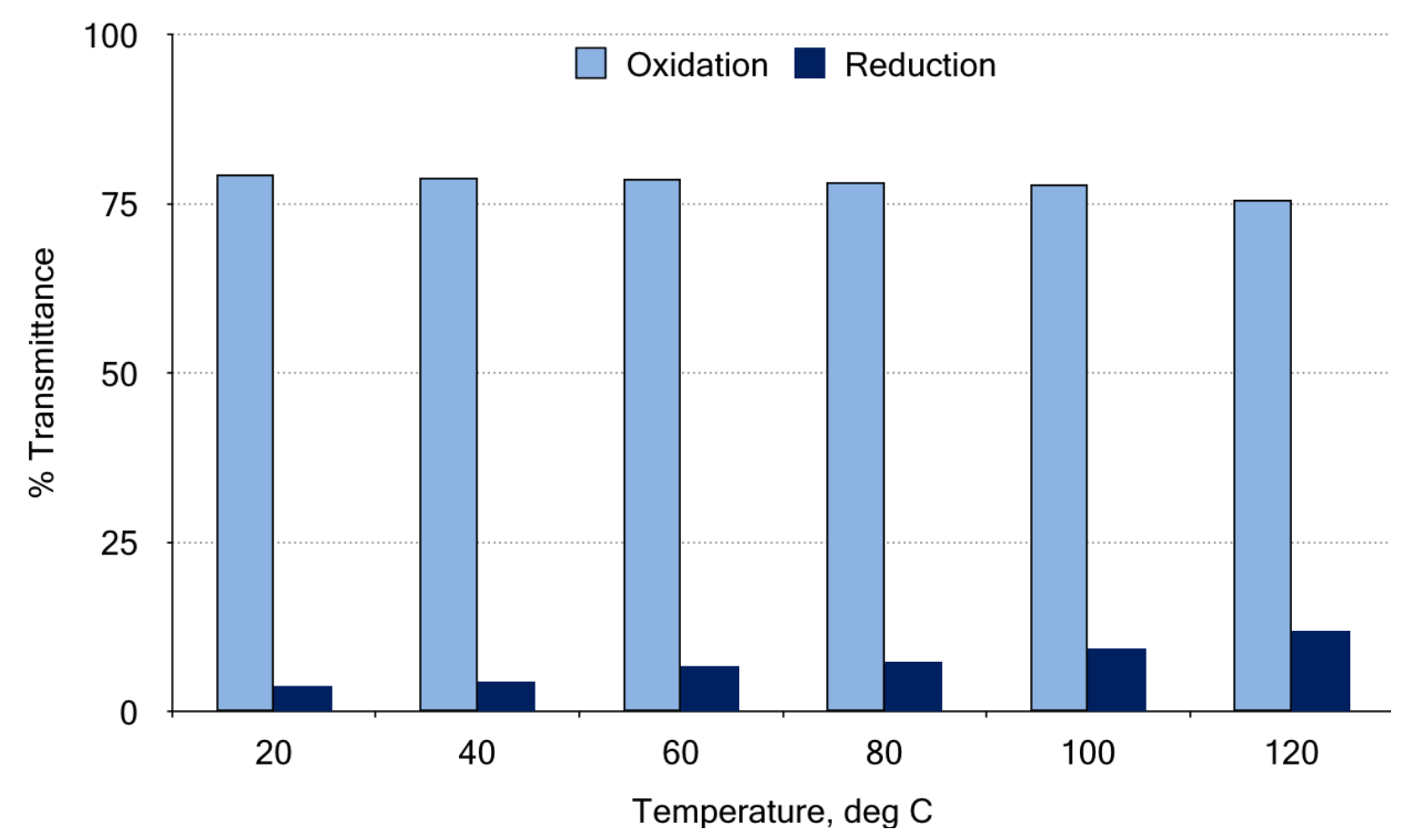

Figure 2.7: Variation of transmittance of PProDOT-Me $\mathrm{M}_{2}$ film from $20^{\circ} \mathrm{C}$ to $120^{\circ} \mathrm{C}$ in the oxidized and neutral state.

in the oxidized state and the transmittance increases by about $8 \%$ from $20{ }^{\circ} \mathrm{C}$ to 120 ${ }^{\circ} \mathrm{C}$ in the neutral state.

The difference in change of transmittance between the oxidized and neutral state arises from the differences in structural stability of the aromatic( neutral state ) and quinoid( oxidized form) structure. At the first glance ProDOT-Me $\mathrm{M}_{2}$ appears to be a simply substituted polythiophene. The ProDOT-Me $\mathrm{M}_{2}$ has reduced band gap compared to polythiophene, which can be attributed to electron donating alkoxy group attached at the $3^{\text {rd }}$ and $4^{\text {th }}$ positions of the polythiophene ring. The ground state of ProDOT-Me $\mathrm{Me}_{2}$ is expected to be neutral. However, from the spectroscopy data, based on Raman peaks corresponding to the stretching of $C \alpha-C \beta$ bonds shifts to higher wavenumbers upon doping suggesting an increase in electron density. Hence the character of the bond evolves from single to double[53]. These observations 
suggest that quinoid structure is more stable than the aromatic structure. Therefore, the oxidized state of ProDOT-Me $\mathrm{M}_{2}$ film is more stable than the neutral state which is reflected in the difference in change of transmittance over temperature.

The decrease in transmittance in of the oxidized ProDOT-Me $\mathrm{M}_{2}$ film with increase in temperature can be due to the temperature induced phase transition of the alkoxy group attached at the $3^{\text {rd }}$ and $4^{\text {th }}$ position of the conjugated backbone resulting in decreasing conjugated length. The decrease in conjugation length leads to hypsochromic effect whereby the absorption peak moves to a smaller wavelength compared to the previous state.The increase in the transmittance of the neutral ProDOT- $\mathrm{Me}_{2}$ film may be attributed to bathochromic effect. The absorption peak slightly shifts to longer wavelength causing a slight increase in transmittance.

\section{$\mathrm{V}_{2} \mathrm{O}_{5}-\mathrm{TiO}_{2}$ film as counter electrode}

The choice of counter electrode material is critical in window application since it should have high ionic capacitance and transparency. ProDOT-Me ${ }_{2}$ film exhibits a fast switching speed of a few seconds, therefore, the counter electrode must be capable of absorbing ions equivalent to the discharge of the working electrode material and the rate of absorption should be sufficient to allow maximum discharge rate of the electrochromic material.

Vanadium oxides have been thoroughly investigated for their ionic storage properties as a counter-electrode in batteries and, more recently, their suitability as a counter-electrode for inorganic ECDs[3]. The layered morphology of Vanadium pentoxide $\left(\mathrm{V}_{2} \mathrm{O}_{5}\right)$ promotes the intercalation of $\mathrm{Li}^{+}$ions, shown below,

$$
V_{2} O_{5}+x e^{-}+x L i^{+} \Leftrightarrow L i_{x} V_{2} O_{5}
$$

However vanadium oxides tend to display adverse coloration upon lithium insertion and extraction, depending on film thickness, ranging from light yellow to orange in the oxidized state to dark green and black in the reduced state. The lack of a visibly transparent state is detrimental to vanadium oxides use in optical devices and limits the thickness of the film that can be employed. Many of the optical and electrical properties of vanadium oxide thin films deposited under RF and DC sputtering and 
evaporation are well characterized. Sol-gel processes have been explored at a less comprehensive level, but show great potential and are of great interest due to the relative low cost of equipment involved and process flexibility. In this study, sol-gel processing was used to develop $\mathrm{V}_{2} \mathrm{O}_{5}-\mathrm{TiO}_{2}$ film with $300 \mathrm{~nm}$ thickness.

Solution preparation involved, synthesis of $\mathrm{V}_{2} \mathrm{O}_{5} \cdot n \mathrm{H}_{2} \mathrm{O}$ using method developed by Takahashi et al [54]. Crystalline $\mathrm{V}_{2} \mathrm{O}_{5}$ powder was dissolved in a Hydrogen peroxide solution at a molar ratio of 8:1 $\left(\mathrm{H}_{2} \mathrm{O}_{2}\right.$ to $\left.\mathrm{V}_{2} \mathrm{O}_{5}\right)$. The ensuing reaction results in the breakdown of $\mathrm{H}_{2} \mathrm{O}_{2}$ and $\mathrm{V}_{2} \mathrm{O}_{5}$ which reform as $\mathrm{VO}^{2+}$ clusters and $\mathrm{V}(\mathrm{V})$ peroxo complexes. This $\mathrm{VO}^{2+}$ solution appears as a transparent orange solution and changes to a transparent dark red solution after 1 hour of vigorous stirring. Sonicating the solution for 2 hours creates a dark red/brown gel that, studies have shown, leads to $\mathrm{V}_{2} \mathrm{O}_{5} \cdot n \mathrm{H}_{2} \mathrm{O}$ layers upon drying. The gel was then dispersed into water creating a dark red transparent solution having a vanadium ion concentration of $0.005 \mathrm{~mol} / 1$ and $\mathrm{pH} \approx 2.7$. Thin films were deposited onto an ITO ( $6 \Omega$ resistance $/ \mathrm{in}^{2}$, $76 \mathrm{~mm} \times 76 \mathrm{~mm}$ ) substrate submerged in the $\mathrm{V}_{2} \mathrm{O}_{5}$ solution in a three electrode cell and subjected to chronoamperometry technique (CH 1605A, CH Instruments, Electrochemical Analyzer) using a Platinum wire counter electrode and silver wire reference electrode [52]. A voltage of $3 \mathrm{~V}$ and a deposition time of 130 seconds is applied. Excess liquid was removed from the films which were dried in air for 15 minutes and then baked at $110^{\circ} \mathrm{C}$ for 8 hours to remove remaining moisture.

\section{Heat resistance of $\mathrm{V} 2 \mathrm{O} 5-\mathrm{TiO} 2$ film}

The heat resistance of $\mathrm{V}_{2} \mathrm{O}_{5}-\mathrm{TiO}_{2}$ film was tested over a range of temperature of $20{ }^{\circ} \mathrm{C}, 40{ }^{\circ} \mathrm{C}, 60{ }^{\circ} \mathrm{C}, 80{ }^{\circ} \mathrm{C}, 100{ }^{\circ} \mathrm{C}, 120{ }^{\circ} \mathrm{C}$ to measure the progressive variation in transmittance of the film over temperature, shown in Figure 2.8.

The $\mathrm{V}_{2} \mathrm{O}_{5}-\mathrm{TiO}_{2}$ film is relatively stable over a range of temperature, maintaining a transmittance of over $70 \%$ in reduced and oxidized state. The stability of the counter electrode is as expected since it is a metal oxide composite film. 


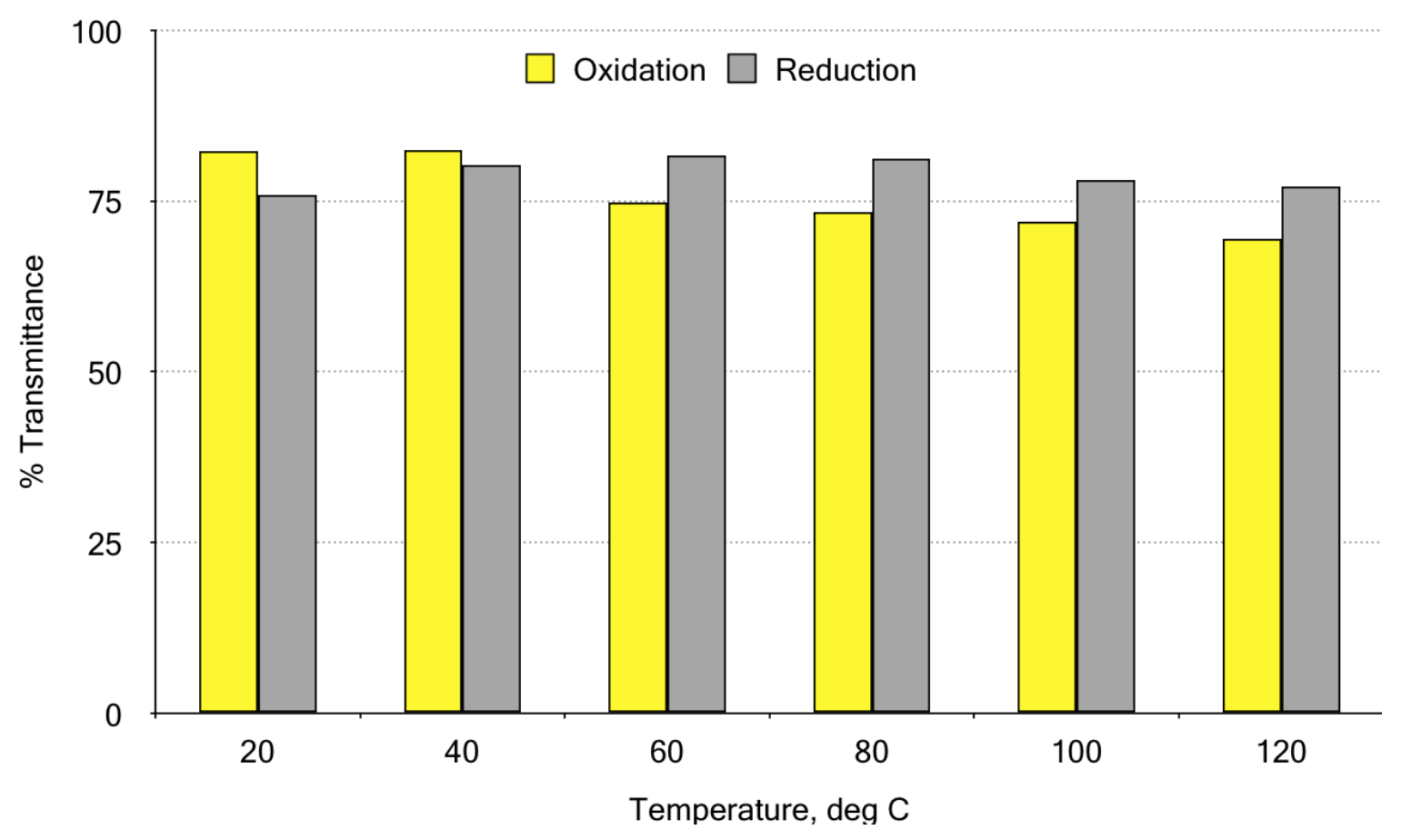

Figure 2.8: Variation of transmittance of PProDOT-Me 2 film from $20{ }^{\circ} \mathrm{C}$ to $120{ }^{\circ} \mathrm{C}$ in the oxidized and reduced state. 
Chapter 3

\section{ELECTROLYTE DEVELOPMENT}

\subsection{Development of electrolyte for ECWs}

The gel electrolyte has generally consists of an alkali metal salt in a polymer host. In addition to having high ionic conductivity and good stability, the electrolytes developed should also have transmittance. Several research papers have investigated polymer solid electrolytes but they tend to have low ionic conductivity. Alternatively, gel electrolytes offer higher conductivity without sacrificing the stability of the electrolyte. In gel electrolytes, the polymer matrix like polyethylene imine(PEI) gives dimensional stability while the high permittivity of the solvents make the movement of Lithium cations easier and faster. The lower viscosity of solvent based gel electrolyte have improved their ionic conductivity.

Electrolytes for ECWs should be highly ionically ionically conductive but the electrolyte should be electrically insulating. High ionic conductivity ensures the movement of cations and anions in the electrolyte. While if the electrolyte is electrically conductive it short circuits the ECW. Transmittance is another important criteria to be taken into consideration while developing electrolytes for ECW.

To develop heat resistant ECW, along with the electrodes the electrolyte should also be thermally stable. The electrolyte should maintain high transparency and ionic conductivity over a range of temperatures. As per the problem statement, the electrolyte should have high ionic conductivity and transmittance from room temperature to $120{ }^{\circ} \mathrm{C}$.

\subsection{Ionic conductivity of electrolyte}

Ionic conductivity is generally given by the equation,

$$
\sigma_{i}=\sum n e \mu
$$



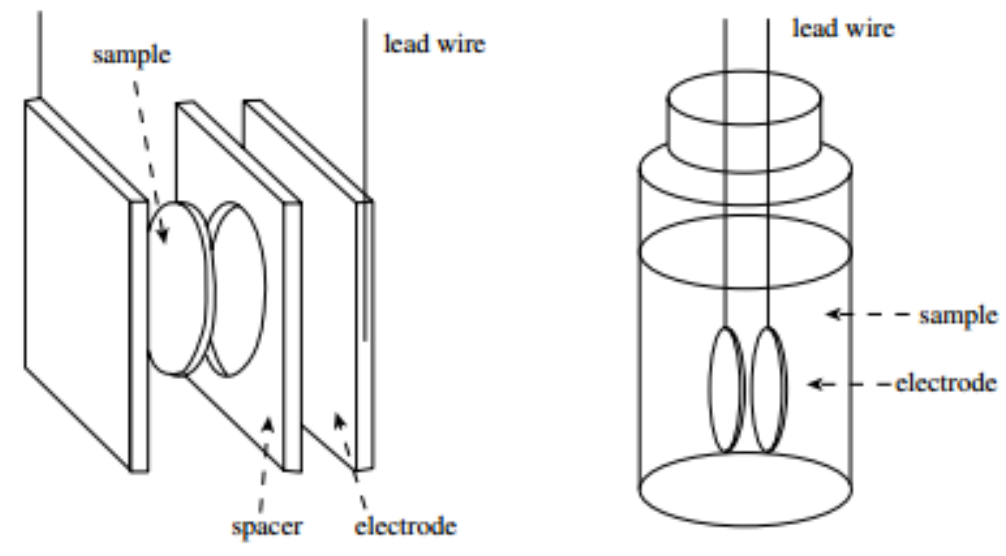

Figure 3.1: Schematic representation of cells used for ionic conductivity measurement by impedance method as an example. (a) Solid-state samples; (b) liquid-state samples[55].

where $n$ is carrier ion number, $e$ is electric charge, and $m$ is mobility of carrier ions.

The ionic conductivity measurement may vary depending on the moisture absorption, experimental environment and electrode species. Therefore, measurements have to be performed with consistent to get comparable values. Therefore, electrochemical cells were prepared inside the glove box under argon gas. Ionic conductivity is dened as the reciprocal of proper resistance $\left(R_{b}\right)$. It is obtained by calculating,

$$
\sigma_{i}=\frac{l}{R_{b} S}
$$

where $l$ is the distance between the two electrodes and $S$ is the mean area of the electrodes. Since it is almost impossible to measure actual surface area of the applied electrode, $l=S$, which is known as cell constant, is determined by measuring the conductivity of standard solutions like aqueous $\mathrm{KCl}[55]$. For ionic conductivity, $\mathrm{S} / \mathrm{cm}$ or $\mathrm{mS} / \mathrm{cm}$ are used as the unit of measure.

The $R_{b}$ based on the sample cannot be calculated correctly, since the electric charge resistance and electric double layer at the electrode resistance will also be detected. The ionic conductivity of an electrolyte is measured through dc four probe measurement and complex impedance method and is used to separate sample bulk 
and electrode. The complex impedance method has a advantage that it could be performed using both non blocking electrodes( same carrier ion $\mathrm{M}^{n+}$ and metal $\mathrm{M}$ ) and blocking electrodes( generally platinum and silver electrodes are used, the charge cannot be transferred from electrodes to carrier ions. The two probe cell where the sample is sandwiched between two polished and washed electrodes is used in complex impedance measurement, shown in Figure 3.1.

Proper contact between the sample and electrode, the polymer electrolyte if solid should be in the molten form and spacers are used to separate the two electrodes to prevent short circuit.

When using metals we must be aware of the strong reduction ability of metals as non blocking electrodes. Lithium metal should be handled in completely dry atmosphere. It cannot be handled in water or solutions containing active protons. In addition, since lithium nitride is produced by the reaction between lithium metal and nitrogen gas the experiment should be performed under argon gas atmosphere. Generally the ionic conductivity of the activated metal is measured using blocked electrodes method.

The impedance analyzer is frequently used under an alternating current. The reliability of the observed impedance depends on the frequency applied. It decreases with increasing frequency. Measurement frequency range is generally from $10 \mathrm{~Hz}$ to $1 \mathrm{MHz}$, of course depending on the performance of the equipment used. Although about $10 \mathrm{mV}$ amplitude voltage is desirable, it depends on sample, and the appropriate value is chosen according to each sample. When absolute value of impedance is large , sufcient current cannot be obtained under ordinary conditions. However, when the absolute value of the obtained impedance is small, the experimental error becomes large due to large current. These values can be adjusted by changing the samples thickness [58].

\subsection{Polyethylene Imine(PEI) based electrolyte}

In several previous studies alkali metals in oxygen containing polymers such as poly(ethylene oxide), poly(propylene oxide) and poly(ethylene succinate)[56],[57]. It 
is considered that there are strong interactions between the unshared pair of electrons on oxygen and cation. Therefore, in this study poly(ethylene imine) based electrolytes were developed and studied. The lone pair of electrons on the nitrogen atom will form coordinate bond with the lithium cation thus promoting the cation and anion dissociation in the solution.

Generally, alkali metal is dissolved in polymer matrix which acts as a host material. These electrolytes tend to have low ionic conductivity. But gel type electrolytes with solvent have higher ionic conductivity without compromising the stability of the electrolyte. Among the numerous "gel" formulations studied, the gel types can be distinguished as (1) plasticized semicrystalline polymers, where crystal lamellae serve as physical crosslinks (2) two-phase electrolytes where the solvent fills a network of pores in a solid, insoluble polymer scaffold; and (3) solvent-swollen, chemically crosslinked polymer networks[58]. Covalently crosslinked gel electrolytes are attractive because the solvent or plasticizer increases the mobility of the $\mathrm{Li}^{+}$cations, while crosslink junctions maintain rubber-like mechanical behavior. In crosslinked gels, the conductivity is not hampered by the presence of crystalline microdomains as in plasticized semicrystalline solid polymers, and no continuous pathways exist for possible lithium dendrite growth as in porous "gel" electrolytes[59]. However, excessive dilution of crosslinked gel electrolytes also generally degrades mechanical properties, so there exists a trade-off between maximizing conductivity (increasing solvent content) and maintaining desirable mechanical behavior [60].

Among polymer electrolytes developed using different polymer matrices the electrolytes developed with PEI are most promising due to low glass transition temperatures, high ionic conductivity and ease of random crosslinking via primary and secondary amine groups. The amines in the backbone and end groups of PEI coordinate with the $\mathrm{Li}^{+}$cation, reducing the binding energy between $\mathrm{Li}^{+}$and the counter-anion like $\mathrm{ClO}^{4-}$, and increasing the concentration of loosely bound $\mathrm{Li}^{+}$cations available for conduction. Several studies of PEI-based electrolytes have appeared recently. Glatzhofer et al. studied electrolytes of poly(N-(2-(2-methoxyethoxy)ethyl)ethylenimine), a PEOPEI copolymer, which exhibited i as high as $7 \times 10^{-5} \mathrm{~S} \mathrm{~cm}^{-1}$ at $60{ }^{\circ} \mathrm{C}$ [61]. 
Armand et al. have examined solid PEIPEOPEI triblock copolymer electrolytes, which serve as a dual matrix for $\mathrm{Li}^{+}$and $\mathrm{Cu}^{2+}$ ions [62],[63].

\subsubsection{Preparation of heat resistant PEI based electrolytes}

While developing PEI based electrolyte two major requirements were kept in mind, 1) high ionic conductivity before and after heat treatment, 2) high transparancy before and after heat treatment.

Gel type electrolyte was developed with PEI as the polymer matrix, dimethyl sulfoxide(DMSO) as the solvent and lithium perchlorate $\left(\mathrm{LiClO}_{4}\right) /$ Lithium triflate $\left(\mathrm{LiCF}_{3} \mathrm{SO}_{3}\right)$. The solvent of the electrolyte has to be chosen such that it has high dielectric constant and dissolves the polymer and lithium solvent. In this study, DMSO and dimethylformamide(DMF) were first considered due to its capability to completely dissolve PEI. The dielectric constant of the solvent determines the ionic mobility of the cation and the anion, therefore, it has a huge influence on the ionic conductivity. DMSO has a dielectric constant value of 46.68 and DMF has a dielectric constant of 36.7164. Owing to the effect of the solvent in electrolyte ionic conductivity DMSO was considered for further studies.

\section{Development of PEI base electrolyte without crosslinking agent}

Branched Poly(ethylene imine) (PEI), with number average molecular weight of 10,000, was purchased from Sigma Aldrich. PEI was dried under vacuum at $70^{\circ} \mathrm{C}$ for 24 hours to remove traces of water. Anhydrous $\mathrm{DMSO}, \mathrm{LiClO}_{4}$ (metal basis, 99.99\%), $\mathrm{LiCF}_{3} \mathrm{SO}_{3}$ were also purchased from Sigma Aldrich. $\mathrm{LiClO}_{4}$ was dried under vacuum for 4 days before use to remove any traces of water. A solution of PEI and DMSO were first prepared according to the weight ratio mentioned in Table 3.1. The solution was allowed to stir for 5 hours. To this solution lithium salt $\left(\mathrm{LiClO}_{4} / \mathrm{LiCF}_{3} \mathrm{SO}_{3}\right)$ was added and allowed to stir for 3 hours. The weight ratio of chemicals added to the solution are listed in Table 3.1,

In ECW application it is critical for the electrolyte to be gel type and have optimum viscosity in order to not flow within or leak out of the ECW. Therefore, 


\begin{tabular}{|c|c|}
\hline Electrolyte No. & $\begin{array}{c}\text { Weight Ratio } \\
\text { PEI/DMSO } /\left(\mathrm{LiClO}_{4}\right) \text { or }\left(\mathrm{LiCF}_{3} \mathrm{SO}_{3}\right)\end{array}$ \\
\hline 1 & $\begin{array}{c}100 / 114.5 / 6.04 \\
\left(\mathrm{LiCF}_{3} \mathrm{SO}_{3}\right)\end{array}$ \\
\hline 2 & $\begin{array}{c}100 / 114.5 / 12.1 \\
\left(\mathrm{LiCF}_{3} \mathrm{SO}_{3}\right)\end{array}$ \\
\hline 3 & $\begin{array}{c}100 / 110 / 1.50 \\
\left(\mathrm{LiClO}_{4}\right)\end{array}$ \\
\hline 4 & $\begin{array}{c}100 / 110 / 3.50 \\
\left(\mathrm{LiClO}_{4}\right)\end{array}$ \\
\hline 5 & $\begin{array}{c}100 / 110 / 5.32 \\
\left(\mathrm{LiClO}_{4}\right)\end{array}$ \\
\hline 6 & $\begin{array}{c}100 / 66 / 1.50 \\
\left(\mathrm{LiClO}_{4}\right)\end{array}$ \\
\hline 7 & $\begin{array}{c}50 / 55 / 3.00 \\
\left(\mathrm{LiClO}_{4}\right)\end{array}$ \\
\hline 8 & $\begin{array}{c}50 / 55 / 1.50 \\
\left(\mathrm{LiClO}_{4}\right)\end{array}$ \\
\hline
\end{tabular}

Table 3.1: Weight ratio of PEI, DMSO and $\mathrm{LiClO}_{4}$ or $\mathrm{LiCF}_{3} \mathrm{SO}_{3}$ in developed electrolytes. 
different concentrations of polymer matrix to solvent from electrolyte number 1 to 8 have been tried. The lithium cations form coordinate bonds with lone pair of electrons on nitrogen atom. Therefore, different concentrations of lithium salt and polymer matrix were also tested for thermal stability.

The developed electrolytes were tested for thermal stability. The electrolyte had to maintain high ionic conductivity and transmittance before and after heat treatment. The electrolytes were sandwiched between two bare ITO glass substrates( 38 $\mathrm{mm} \times 38 \mathrm{~mm}$ ) with a parafilm spacer. The sample was sealed with high temperature resistant epoxy adhesive. The samples were tested at $20^{\circ} \mathrm{C}, 40{ }^{\circ} \mathrm{C}, 60{ }^{\circ} \mathrm{C}, 80{ }^{\circ} \mathrm{C}$, $100{ }^{\circ} \mathrm{C}, 120{ }^{\circ} \mathrm{C}$. Until $100{ }^{\circ} \mathrm{C}$ the samples were tested in environmental chamber and $120{ }^{\circ} \mathrm{C}$ was tested on a hot plate since the maximum attainable temperature in the environment chamber is $100{ }^{\circ} \mathrm{C}$. The transmittance of the electrolytes from 1 to 8 at different temperatures were measured and plotted, shown in Figure 3.2.

From Figure 3.2 it is clear that the developed electrolytes 1 to 8 had a reasonably high transmittance of over $65 \%$ at all temperatures. For ECW application a transmittance of over $70 \%$ at all temperatures is preferred. In order to maintain the performance of the ECW even at high temperatures of $120{ }^{\circ} \mathrm{C}$ the transmittance of the electrolyte layer should be consistent and not drop below $70 \%$. In this case, electrolyte 8 is the best fit for the requirement. The maximum drop observed in electrolyte is was about 3 to $4 \%$ even at high temperature and the transmittance is always maintained above $70 \%$. The electrolyte 8 contains optimized ratio of $\mathrm{PEI} / \mathrm{DMSO} / \mathrm{LiClO}_{4}: 50 / 55 / 1.5$. The ratio of PEI to DMSO was important to maintain the gel like nature and viscosity of the electrolyte. Electrolyte 1 and 2 has lower weight ratio of $\mathrm{PEI} / \mathrm{DMSO}(0.873)$ and therefore has lower viscosity due to higher solvent concentration. Electrolyte 6 has higher weight ratio of PEI/DMSO(1.515). Electrolytes 3, 4, 5 and 7 have the same PEI/DMSO weight ratio but have different salt concentrations. When lithium salt is added to PEI, the primary and secondary amines in the polymer chain form coordinate bond with lithium cation thereby aiding in dissociating the salt. The coordinate bond formed between the cation and the primary and secondary amine is believed to provide thermal stability to the electrolyte. 


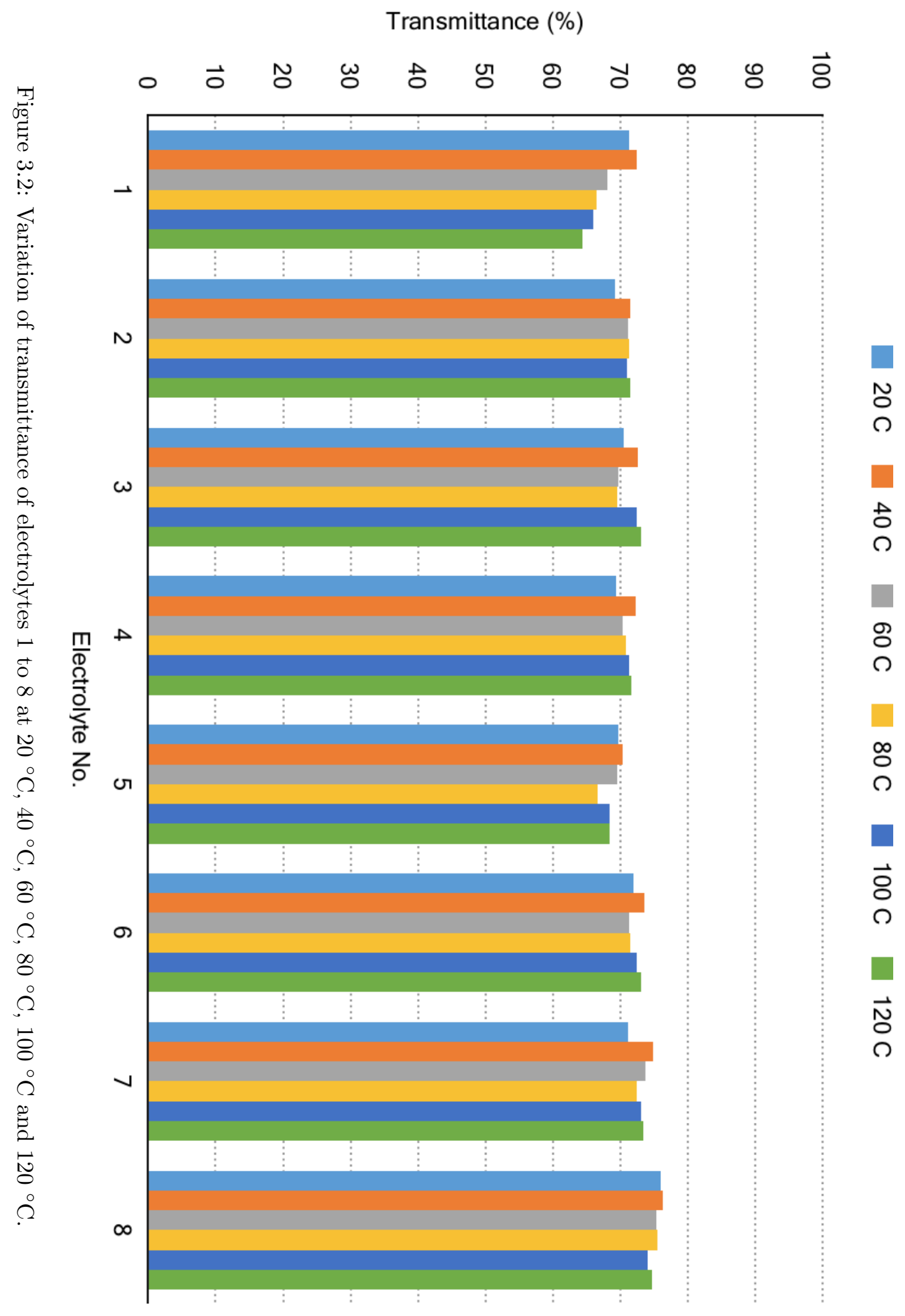


A covalent bond in which one of the two atoms bonded supplies all of the shared electrons.

Coordinate covalent bonds are formed when one atom does not have a complete outer shell, while another atom does have a complete outer shell and has at least one unshared electron pair. Once formed, coordinate covalent bonds are indistinguishable from normal covalent bonds [65]. Therefore, coordinate bonds have equal strength as covalent bonds.

The ionic conductivity of these electrolytes were tested at $20{ }^{\circ} \mathrm{C}, 40{ }^{\circ} \mathrm{C}, 60{ }^{\circ} \mathrm{C}, 80$ ${ }^{\circ} \mathrm{C}, 100{ }^{\circ} \mathrm{C}$ and $120{ }^{\circ} \mathrm{C}$. Ideally, the ionic conductivity of the electrolyte should not vary considerably with temperature in order to maintain consistency of performance of ECW. Figure 3.3 shows the ionic conductivity of the developed electrolytes over a range of temperature. It can be observed that the ionic conductivity of the electrolyte remains stable from $20{ }^{\circ} \mathrm{C}$ to $100{ }^{\circ} \mathrm{C}$. This can be attributed to the presence of coordinate bond between the lithium cations and nitrogen atoms in primary and secondary amine.

The chemical stability of the developed electrolytes were checked through Differential Scanning Calorimetry(DSC) measurements. The DSC measurements were performed in the expected ECW operation temperature range from $20{ }^{\circ} \mathrm{C}$ to $130{ }^{\circ} \mathrm{C}$, shown in Figure 3.4. The program was written to hold the sample at $20{ }^{\circ} \mathrm{C}$ for 2 minutes and increase the temperature by $5{ }^{\circ} \mathrm{C}$ per minute until the sample reached $130{ }^{\circ} \mathrm{C}$ and the sample was maintained at $130{ }^{\circ} \mathrm{C}$ for 30 minutes. In DSC measurements, the phase transformation of the sample is indicated by a narrow peak at the temperature at which the phase change occurs. From Figure 3.4 we can observe that there are no obvious peaks in the operating temperature range. This implies that the sample does not undergo phase transformation which may lead to degradation of critical properties like mechanical stability, transmittance, viscosity and ionic conductivity. Therefore, we can conclude that the DSC measurements confirm the stability of the electrolyte in the operating temperature range. We can observe that compared to all the other electrolytes the DSC measurements of electrolyte no. 5 and 6 are almost parallel to the horizontal axis. This indicates electrolyte 5 and 6 


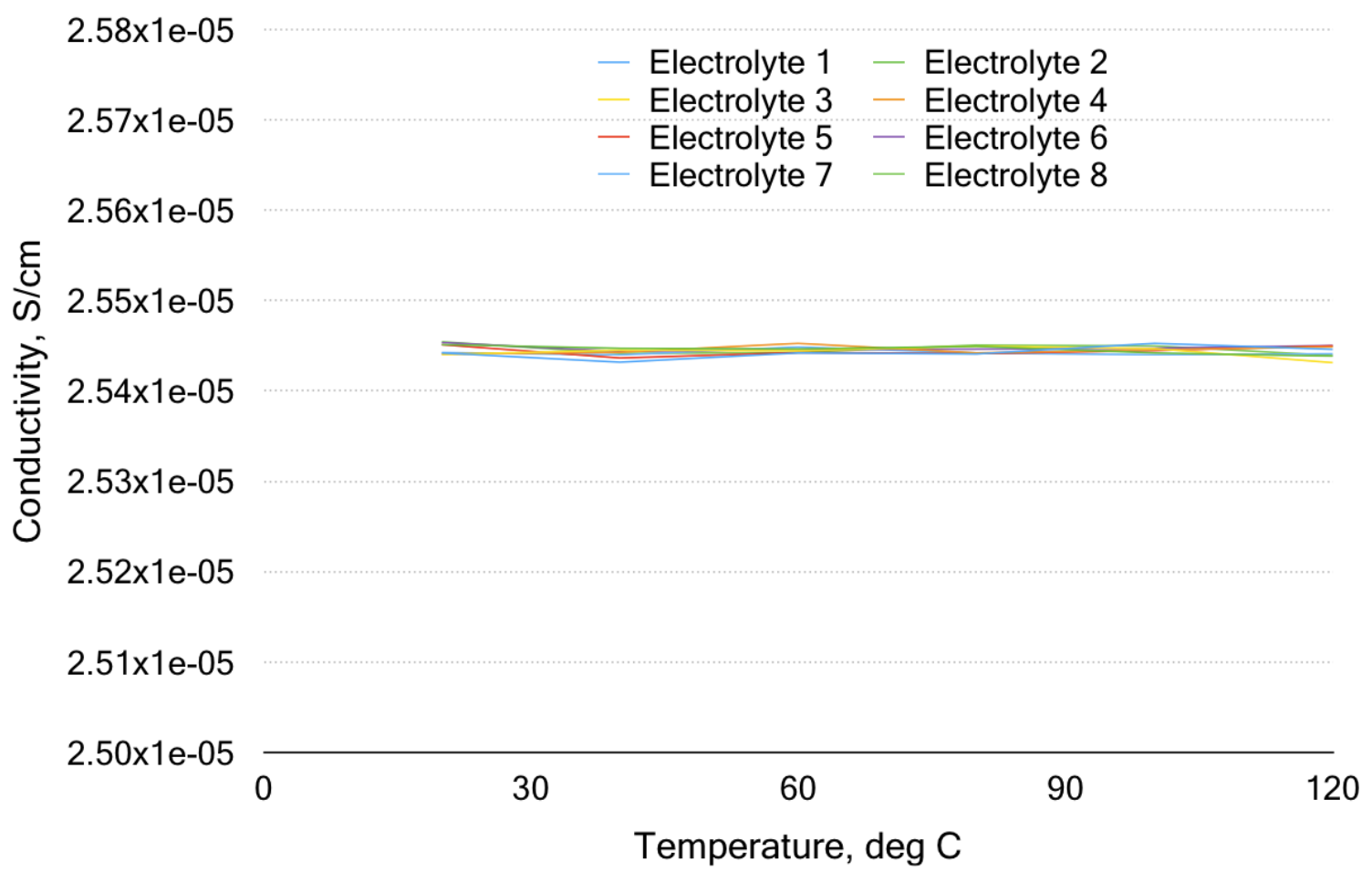

Figure 3.3: Stability of ionic conductivity of electrolytes 1 to 8 at $20{ }^{\circ} \mathrm{C}, 40{ }^{\circ} \mathrm{C}, 60{ }^{\circ} \mathrm{C}, 80$ ${ }^{\circ} \mathrm{C}, 100{ }^{\circ} \mathrm{C}$ and $120{ }^{\circ} \mathrm{C}$.

are the most thermally stable compared to the other electrolytes. This can be attributed to high concentration of lithium salt in the electrolyte. Higher the lithium salt concentration higher is the number of coordinate bond present in the electrolyte which provides better thermal stability to the electrolyte.

\section{Development of PEI baes electrolyte with crosslinking agent}

Branched Poly(ethylene imine) (PEI), with number average molecular weight of 10,000 was purchased from Sigma Aldrich. PEI was dried under vacuum at $70{ }^{\circ} \mathrm{C}$ for

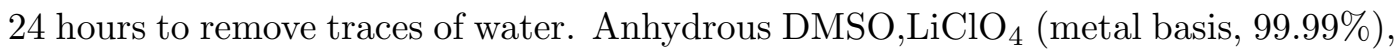
$\mathrm{LiCF}_{3} \mathrm{SO}_{3}$ were also purchased from Sigma Aldrich. $\mathrm{LiClO}_{4}$ was dried under vacuum for 4 days before use to remove any traces of water. A solution of PEI and DMSO were first prepared according to the weight ratio mentioned in Table 3.2. The solution 


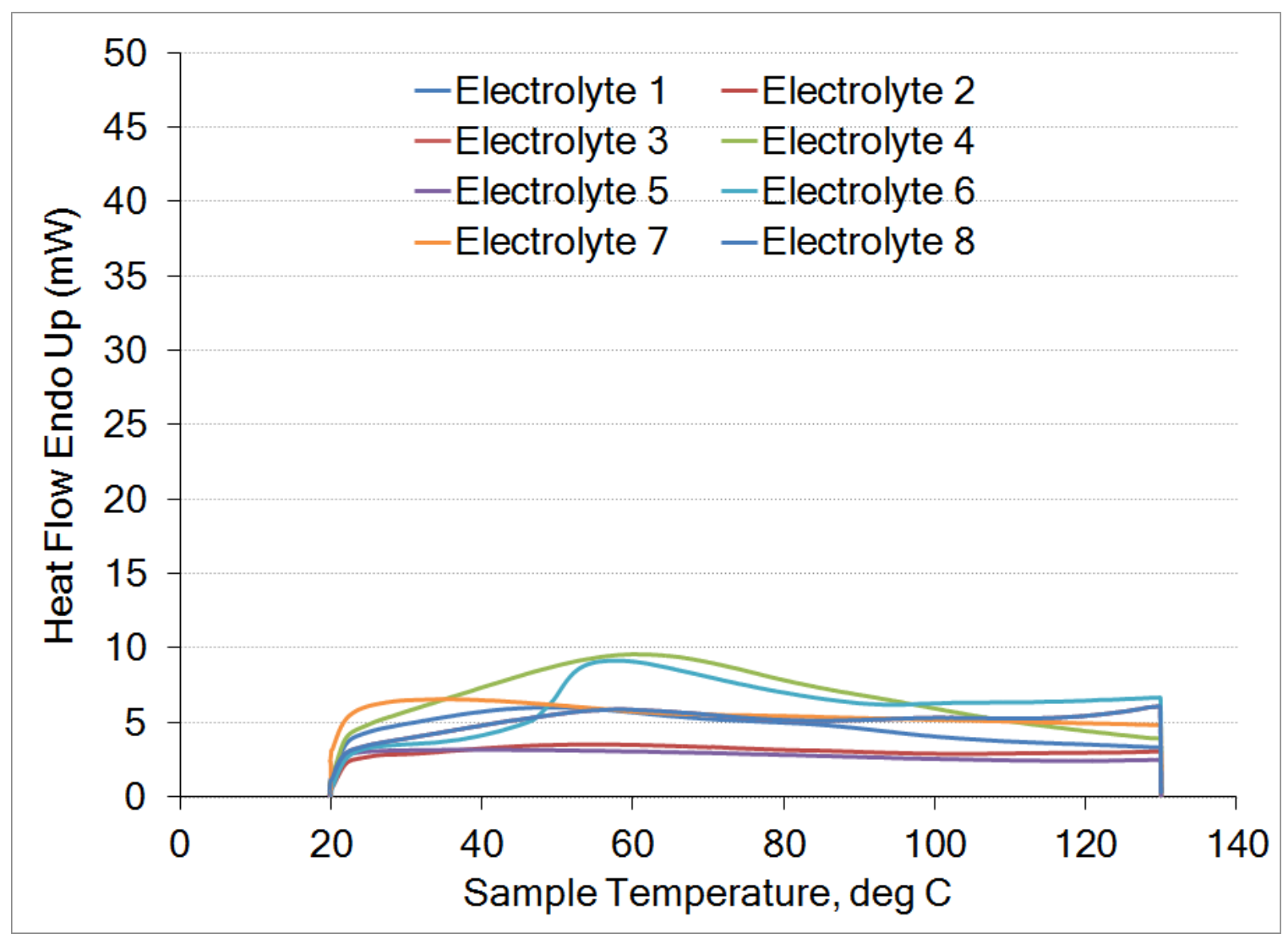

Figure 3.4: DSC measurements of electrolytes 1 to 8 from $20{ }^{\circ} \mathrm{C}$ to $130{ }^{\circ} \mathrm{C}$

was allowed to stir for 5 hours. To this solution lithium salt $\left(\mathrm{LiClO}_{4} / \mathrm{LiCF}_{3} \mathrm{SO}_{3}\right)$ was added and allowed to stir for 3 hours. The crosslinking agent Bisphenol A diglycidyl ether(DGEBA) was added to the electrolyte in varying amounts, shown in Table 3.2. The electrolyte was allowed to cross link for 24 hours before use. The concentration of DGEBA was gradually increased to measure its effect on the transmittance and ionic conductivity of the electrolyte.

Random crosslinking of PEI in solution is an attractive and economical processing strategy to produce rubber-like electrolytes, an idea first pursued by Glatzhofer et al., who crosslinked PEI by a Michael addition reaction between acrylates and the amines of PEI [65]. The addition of crosslinking agent to the electrolyte makes the electrolyte more viscous. The viscosity of the electrolyte should be optimized for use 


\begin{tabular}{|c|c|}
\hline Electrolyte No. & $\begin{array}{c}\text { Weight Ratio } \\
\text { PEI/DMSO/DGEBA/( }\left(\mathrm{LiClO}_{4}\right) \text { or }\left(\mathrm{LiCF}_{3} \mathrm{SO}_{3}\right)\end{array}$ \\
\hline 9 & $\begin{array}{c}100 / 114.5 / 4.16 / 12.1 \\
\left(\mathrm{LiCF}_{3} \mathrm{SO}_{3}\right)\end{array}$ \\
\hline 10 & $\begin{array}{c}50 / 44 / 0.8 / 1.50 \\
\left(\mathrm{LiClO}_{4}\right)\end{array}$ \\
\hline 11 & $\begin{array}{c}50 / 44 / 1.02 / 1.50 \\
\left(\mathrm{LiClO}_{4}\right)\end{array}$ \\
\hline 12 & $\begin{array}{c}50 / 55 / 2.00 / 1.50 \\
\left(\mathrm{LiClO}_{4}\right)\end{array}$ \\
\hline 13 & $\begin{array}{c}50 / 55 / 4.00 / 1.50 \\
\left(\mathrm{LiClO}_{4}\right)\end{array}$ \\
\hline 14 & $\begin{array}{c}50 / 55 / 5.00 / 1.50 \\
\left(\mathrm{LiClO}_{4}\right)\end{array}$ \\
\hline 15 & $\begin{array}{c}50 / 55 / 6.00 / 1.50 \\
\left(\mathrm{LiClO}_{4}\right)\end{array}$ \\
\hline 16 & $\begin{array}{c}50 / 55 / 7.00 / 1.50 \\
\left(\mathrm{LiClO}_{4}\right)\end{array}$ \\
\hline
\end{tabular}

Table 3.2: Weight ratio of PEI, DMSO, $\mathrm{LiClO}_{4}$ and DGEBA in developed electrolytes. 
in ECW. For industrial purposes, solid state electrolytes with roll on mass production capability would be preferable. Adding a crosslinking agent to the electrolyte opens up a wide range of possibilities to alter the viscosity and the thermal stability of the electrolyte. The structure of DGEBA is shown in Figure 3.5,

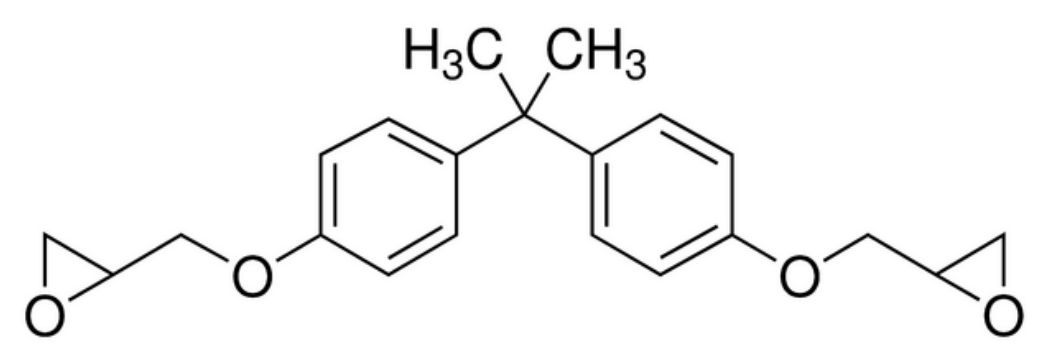

Figure 3.5: Structure of DGEBA [66]

When DGEBA is added to PEI, structure shown in Figure 3.6, standard epoxideamine reaction takes place [60], shown Figure 3.7. This crosslinking reaction improves the thermal stability of the electrolyte.

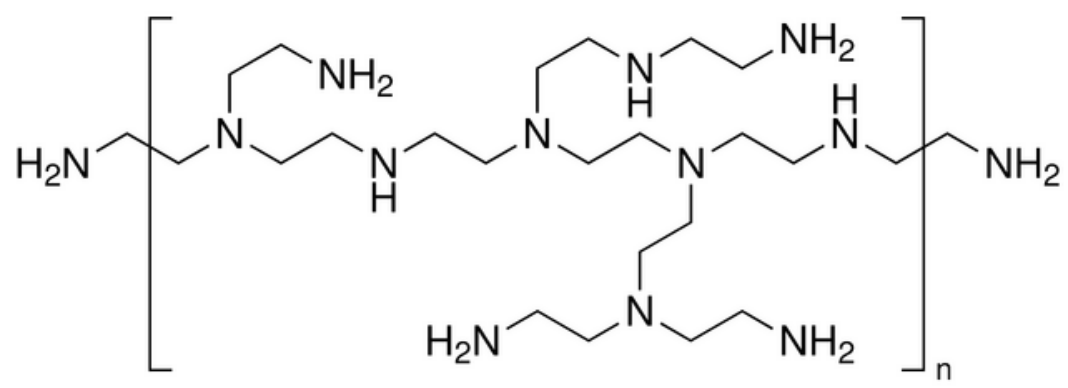

Figure 3.6: Structure of PEI [67]

The electrolytes were sandwiched between two bare ITO glass substrates $(38 \mathrm{~mm}$ $\times 38 \mathrm{~mm}$ ) with a parafilm spacer. The sample was sealed with high temperature resistant epoxy adhesive. The samples were tested at $20^{\circ} \mathrm{C}, 40{ }^{\circ} \mathrm{C}, 60{ }^{\circ} \mathrm{C}, 80^{\circ} \mathrm{C}$, $100{ }^{\circ} \mathrm{C}, 120{ }^{\circ} \mathrm{C}$. Until $100{ }^{\circ} \mathrm{C}$ the samples were tested in environmental chamber and 


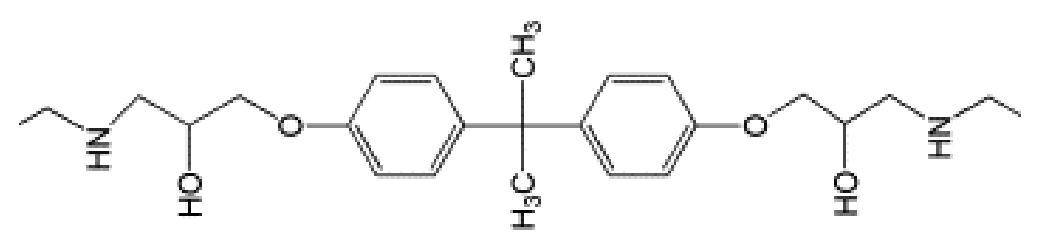

Figure 3.7: Epoxide-amine structure [60]

$120{ }^{\circ} \mathrm{C}$ was tested on a hot plate since the maximum attainable temperature in the environment chamber is $100{ }^{\circ} \mathrm{C}$. The transmittance of the electrolytes from 9 to 16 at different temperatures were measured and plotted, shown in Figure 3.8.

From Figure 3.8, it is evident that as the concentration of DGEBA is increased in the electrolyte the transmittance at room temperature decreases. When the amount of DGEBA added to solution was doubled compared to electrolyte no. 11 the transmittance dropped to $40 \%$ from $70 \%$. When the temperature of the sample increases the transmittance of electrolytes 10 and 11 remain almost constant whereas for electrolytes $12,13,15$ and 16 the transmittance increases. The increase in transmittance for electrolytes $12,13,14,15$ and 16 can be attributed to the reduction in viscosity of the electrolyte and possibly breaking of a few epoxide-amine bonds that make the electrolyte quite similar to electrolytes 9 to 16 . From these set of electrolytes, electrolyte no. 11 suits the ECW window application the best since the transmittance of the electrolyte is consistently above $70 \%$.

The ionic conductivity of these electrolytes were tested at $20{ }^{\circ} \mathrm{C}, 40{ }^{\circ} \mathrm{C}, 60{ }^{\circ} \mathrm{C}$, $80{ }^{\circ} \mathrm{C}, 100{ }^{\circ} \mathrm{C}, 120{ }^{\circ} \mathrm{C}$. Ideally, the ionic conductivity of the electrolyte should not vary considerably with temperature in order to maintain consistency of performance of ECW. Figure 3.9 shows the ionic conductivity of the developed electrolytes over a range of temperature. It can be observed that the ionic conductivity of the electrolyte remains stable from $20^{\circ} \mathrm{C}$ to $120^{\circ} \mathrm{C}$. This can be attributed to the presence of coordinate bond between the lithium cations and nitrogen atoms in primary and secondary amine and also the presence of epoxide-amine crosslinking bond that increases the 


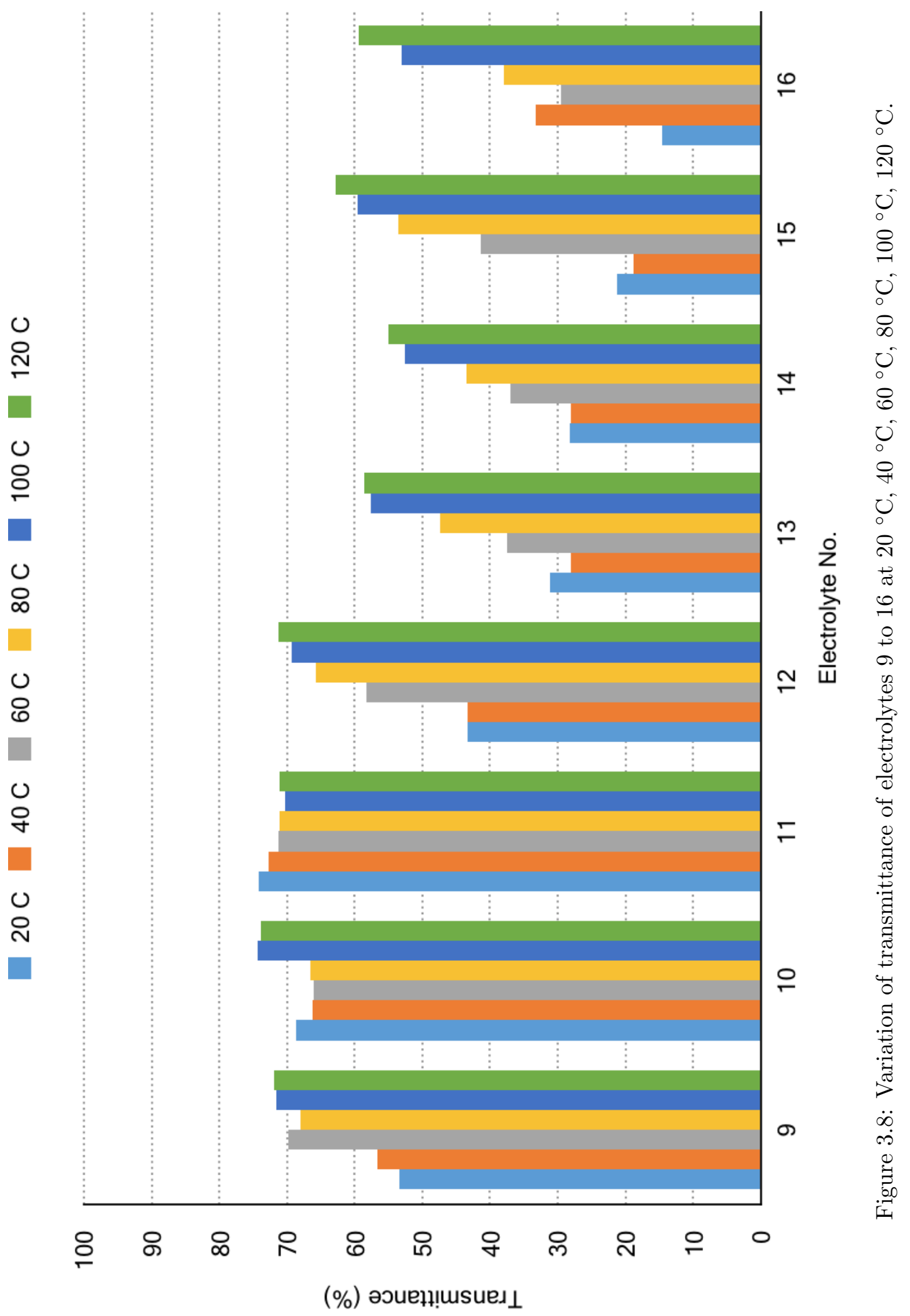




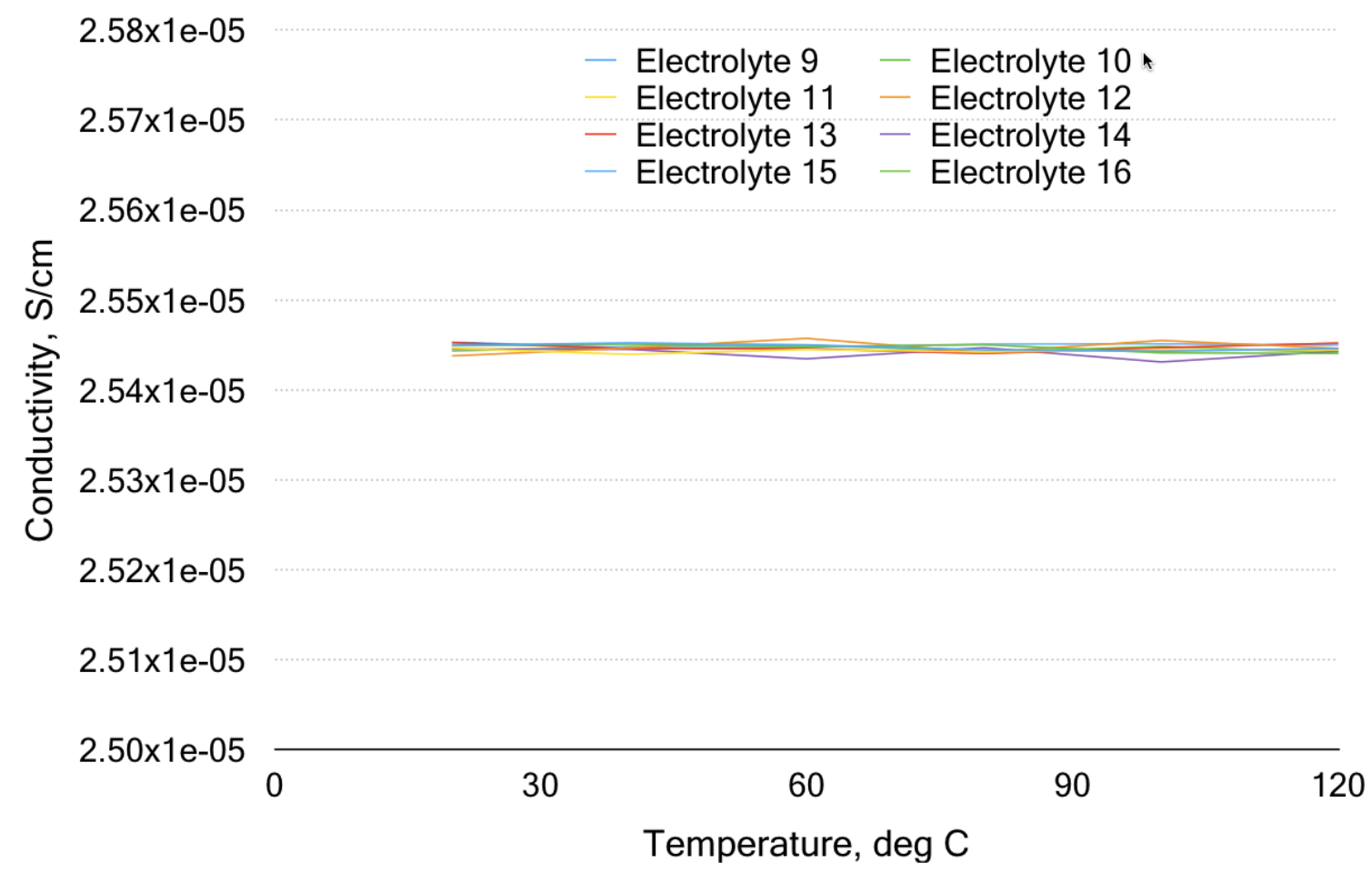

Figure 3.9: Stability of ionic conductivity of electrolytes 9 to 16 at $20^{\circ} \mathrm{C}, 40{ }^{\circ} \mathrm{C}, 60{ }^{\circ} \mathrm{C}, 80$ ${ }^{\circ} \mathrm{C}, 100{ }^{\circ} \mathrm{C}, 120{ }^{\circ} \mathrm{C}$.

thermal stability of the electrolyte.

From Figure 3.9 it can be observed that for all concentrations of DGEBA the ionic conductivity remains the same. This indicates that the lithium ion movement is not affected by the formation of epoxide-amine bond. This is also improves that the ionic conductivity depends on the solvent and not the nature of the crosslinking agent present in the electrolyte.

To establish the chemical stability of the developed electrolytes DSC measurements taken. The DSC measurements were performed in the expected ECW operation temperature range from $20{ }^{\circ} \mathrm{C}$ to $130{ }^{\circ} \mathrm{C}$, shown in Figure 3.10. The program was written to hold the sample at $20{ }^{\circ} \mathrm{C}$ for 2 minutes and increase the temperature by $5{ }^{\circ} \mathrm{C}$ per minute until the sample reached $130{ }^{\circ} \mathrm{C}$ and the sample was maintained at $130{ }^{\circ} \mathrm{C}$ for 30 minutes. In DSC measurements, the phase transformation of the 


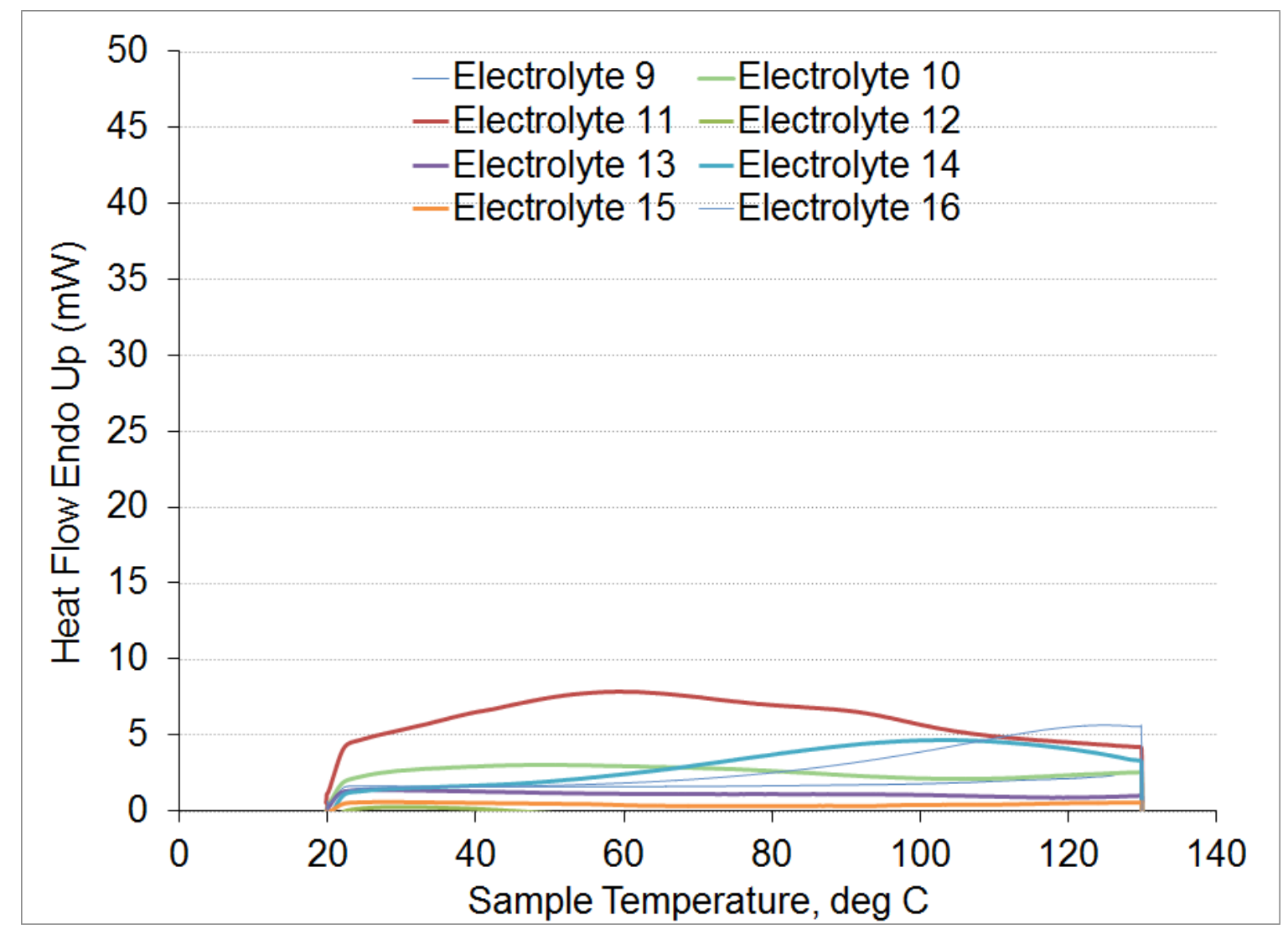

Figure 3.10: DSC measurements of electrolytes 9 to 16 from $20{ }^{\circ} \mathrm{C}$ to $130{ }^{\circ} \mathrm{C}$.

sample is indicated by a narrow peak at the temperature at which the phase change occurs. From Figure 3.10 we can observe that there are no obvious peaks in the operating temperature range.

From DSC measurements of electrolytes 9 to 16 we observe that as concentration of the crosslinking agent, DGEBA increases the electrolyte becomes more thermally stable since the graph becomes almost parallel to the $\mathrm{x}$ axis indicating the thermal stability of the electrolyte. However keeping in mind that transmittance of the electrolyte is critical in electrolytes for window application we can conclude that in this case electrolyte no 11 would best fit the requirements of electrolytes for high temperature resistance ECW. Electrolyte no. 11 shows a consistent transmittance of over $70 \%$ even at higher temperatures, it maintains a high ionic conductivity of 
over $2.543 \times 10^{-5} \mathrm{~S} / \mathrm{cm}$ over all ranges of temperatures and maintains reasonable chemical stability in the operating temperature.

\section{Development of PEI baes electrolyte with UV curable agents}

Branched Poly(ethylene imine) (PEI), with number average molecular weight of 10,000 was purchased from Sigma Aldrich. PEI was dried under vacuum at $70^{\circ} \mathrm{C}$ for 24 hours to remove traces of water. Anhydrous $\mathrm{DMSO}, \mathrm{LiClO}_{4}$ (metal basis, 99.99\%), $\mathrm{LiCF}_{3} \mathrm{SO}_{3}$ were also purchased from Sigma Aldrich. $\mathrm{LiClO}_{4}$ was dried under vacuum for 4 days before use to remove any traces of water. A solution of PEI and DMSO were first prepared according to the weight ratio mentioned in Table 3.2. The solution was allowed to stir for 5 hours. To this solution lithium salt $\left(\mathrm{LiClO}_{4} / \mathrm{LiCF}_{3} \mathrm{SO}_{3}\right)$ was added and allowed to stir for 3 hours. To this electrolyte, UV curable agents polyethylene glycol methyl ether methacrylate(PEGMA), polyethylene glycol molecular weight 200(PEG 200) were added and allowed to stir for 3 hours. Now the reaction initiator 2,2-bis(hydroxymethyl)propionic acid(DMPA) is added to the electrolyte and allowed to stir for 2 hours. The composition of different chemicals are listed in Table 3.3.

\begin{tabular}{|c|c|c|}
\hline Electrolyte No. & $\begin{array}{c}\text { Weight Ratio } \\
\text { (Electrolyte) }\end{array}$ & $\begin{array}{c}\text { Weight Ratio } \\
\text { (UV curable chemicals) } \\
\text { PEGMA/PEG200/DMPA }\end{array}$ \\
\hline \hline 17 & $50 / 55 / 1.5$ & $11.2 / 39.2 / 0.112$ \\
\hline 18 & $50 / 55 / 1.5$ & $3.2 / 11.1 / 0 / 03$ \\
\hline 19 & $50 / 55 / 3.00$ & $11.2 / 39.2 / 0.112$ \\
\hline 20 & $50 / 55 / 3.00$ & $3.2 / 11.1 / 0 / 03$ \\
\hline
\end{tabular}

Table 3.3: Weight ratio of PEI, DMSO, $\mathrm{LiClO}_{4}$, PEGMA, PEG 200 and DMPA in developed electrolytes 
PEGMA, PEG 200 and DMPA, structures shown in Figures 3.11, 3.12 and 3.13 respectively, initiate crosslinking reaction upon irradiation of UV light. The formation of crosslinks upon UV reaction is expected to give better thermal stability to the electrolytes.

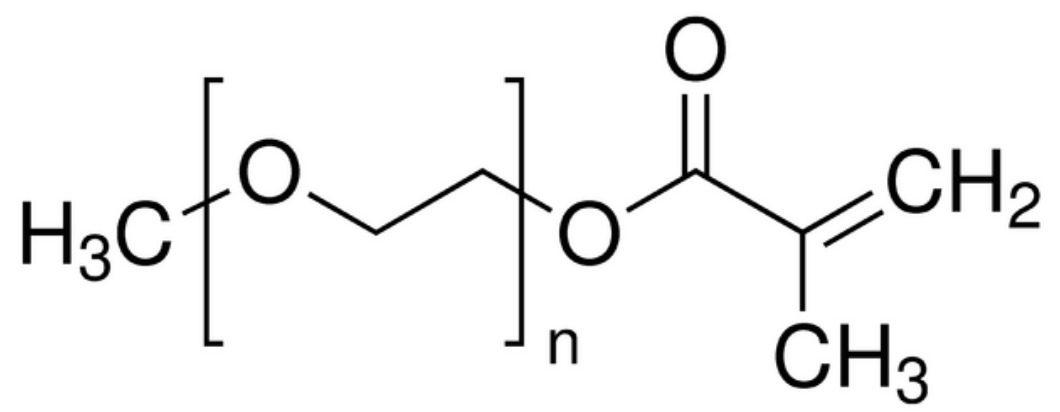

Figure 3.11: Structure of PEGMA [68]

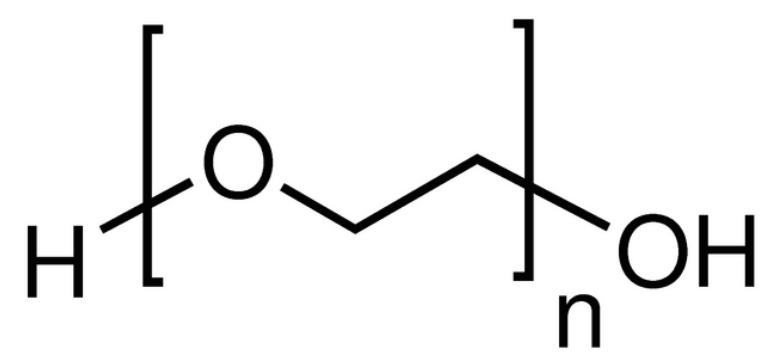

Figure 3.12: Structure of PEG 200 [69]

The electrolytes were sandwiched between two bare ITO glass substrates( $38 \mathrm{~mm}$ $\times 38 \mathrm{~mm}$ ) with a parafilm spacer. The sample was sealed with high temperature resistant epoxy adhesive. The samples were then cured under UV light for 20 minutes on each side of the sample. The samples were tested at $20^{\circ} \mathrm{C}, 40{ }^{\circ} \mathrm{C}, 60{ }^{\circ} \mathrm{C}, 80{ }^{\circ} \mathrm{C}$, $100{ }^{\circ} \mathrm{C}, 120{ }^{\circ} \mathrm{C}$. Until $100{ }^{\circ} \mathrm{C}$ the samples were tested in environmental chamber and $120{ }^{\circ} \mathrm{C}$ was tested on a hot plate since the maximum attainable temperature in the environment chamber is $100{ }^{\circ} \mathrm{C}$. The transmittance of the electrolytes from 17 to 20 at different temperatures were measured and plotted, shown in Figure 3.14. 


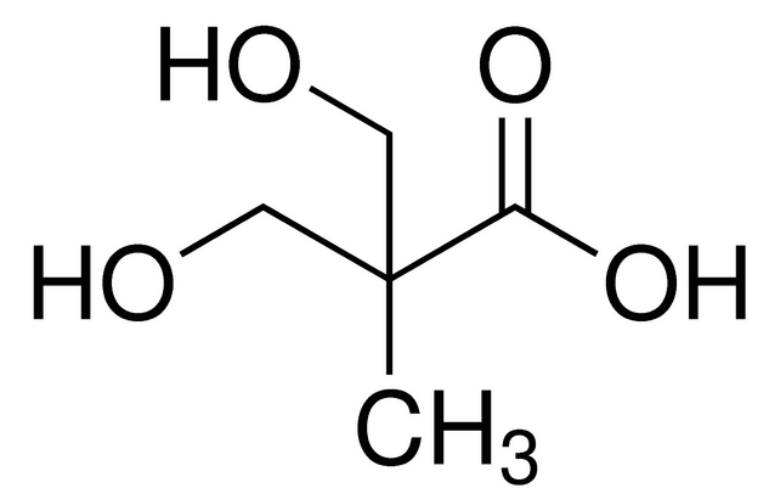

Figure 3.13: Structure of DMPA [70]

From Figure 3.14, we can observe that the transmittance of the four electrolytes remain almost constant over the range of temperature measured. It can be observed that when the concentration of the UV crosslinking agent is higher the transmittance is slightly lower, electrolytes 17 and 19. This may be due to higher crosslinking in the presence of UV that reduces the transmittance of the electrolyte layer sandwiched between two ITO glasses. In this case, electrolyte 18 is the best fit since it has a transmittance of over $70 \%$ at all temperatures. This would ensure consistent performance of the ECW even at elevated temperatures.

The ionic conductivity of these electrolytes were tested at $20{ }^{\circ} \mathrm{C}, 40{ }^{\circ} \mathrm{C}, 60{ }^{\circ} \mathrm{C}, 80$ ${ }^{\circ} \mathrm{C}, 100{ }^{\circ} \mathrm{C}, 120{ }^{\circ} \mathrm{C}$. Ideally, the ionic conductivity of the electrolyte should not vary considerably with temperature in order to maintain consistency of performance of ECW. Figure 3.15 shows the ionic conductivity of the developed electrolytes over a range of temperature. It can be observed that the ionic conductivity of the electrolyte remains stable from $20{ }^{\circ} \mathrm{C}$ to $120{ }^{\circ} \mathrm{C}$. This can be attributed to the presence of coordinate bond between the lithium cations and nitrogen atoms in primary and secondary amine and also the presence of crosslinking bonds formed by the UV curable agents. The UV curable provides comparable stability to the electrolyte compared to the crosslinking agent in terms of transmittance.

The ionic conductivity of electrolytes 17 to 20 is high and stable over a range 


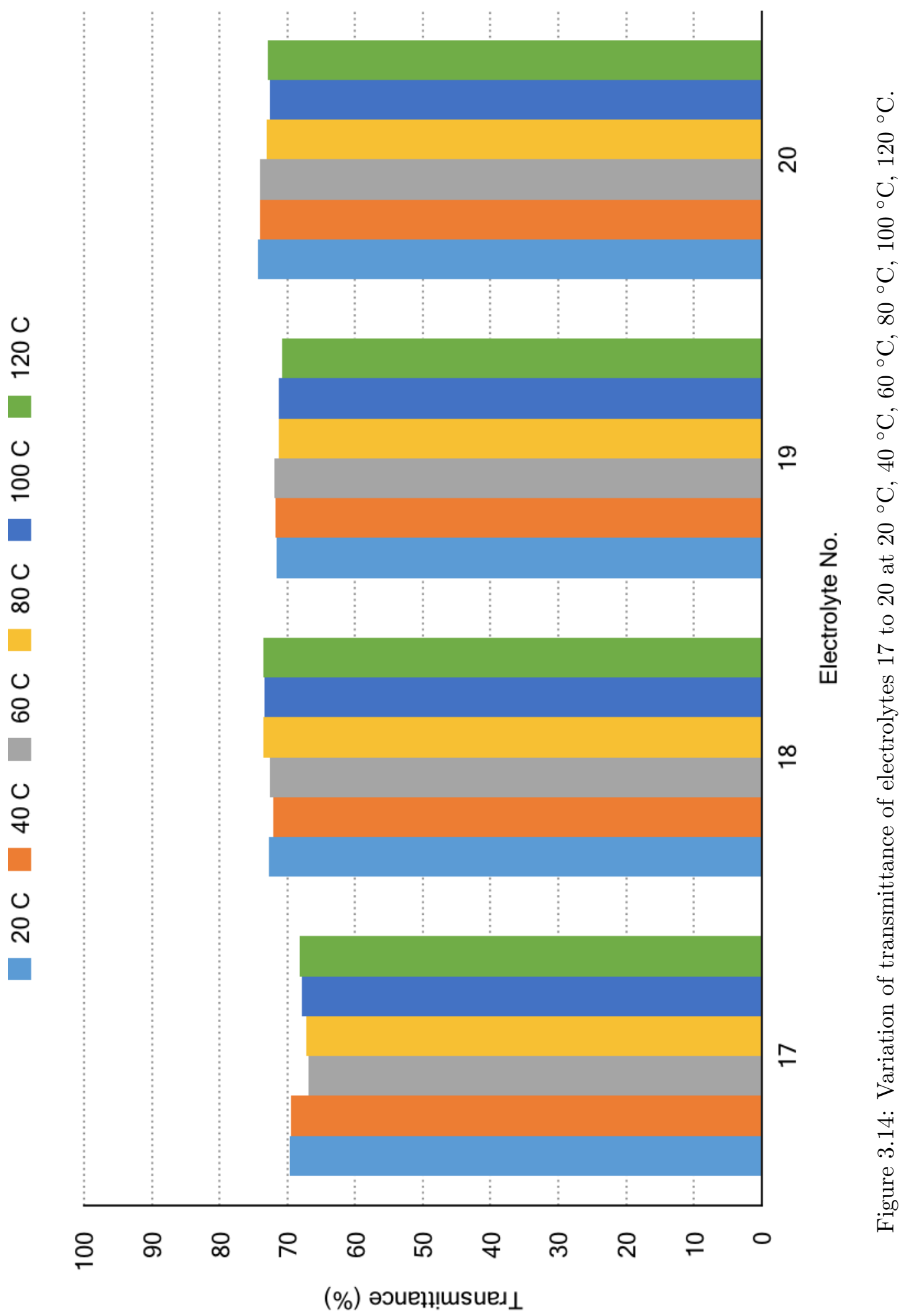




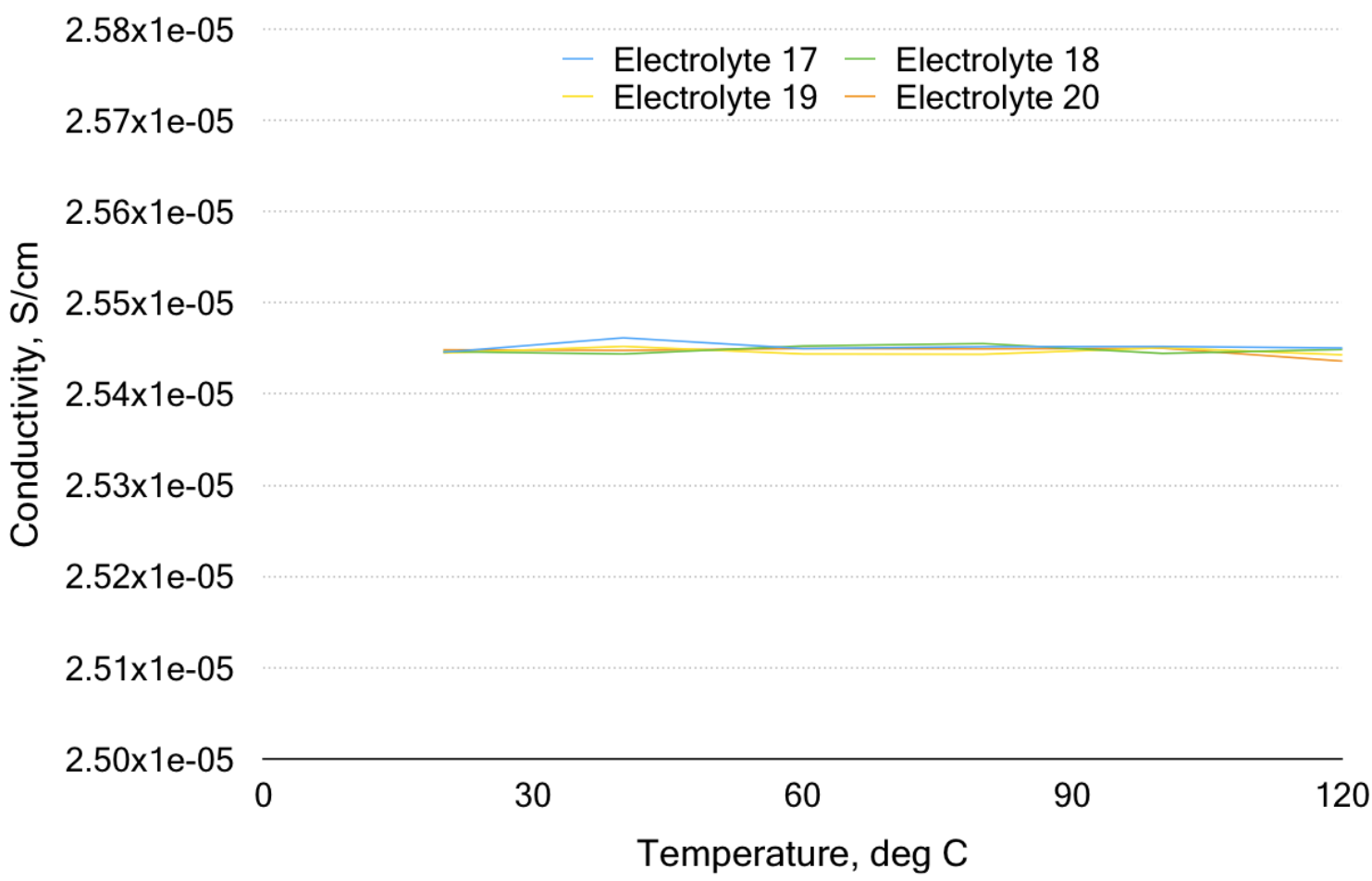

Figure 3.15: Stability of ionic conductivity of electrolytes 17 to 20 at $20{ }^{\circ} \mathrm{C}, 40{ }^{\circ} \mathrm{C}, 60{ }^{\circ} \mathrm{C}$, $80{ }^{\circ} \mathrm{C}, 100{ }^{\circ} \mathrm{C}, 120{ }^{\circ} \mathrm{C}$.

of temperature. This indicates that the presence of crosslinks formed by PEGMA, PEG 200 and DMPA does not affect the mobility of lithium cations even at high temperatures, therefore, the ionic conductivity remains the same irrespective of the addition of UV curable agents.

To establish the chemical stability of the developed electrolytes DSC measurements taken. The DSC measurements were performed in the expected ECW operation temperature range from $20^{\circ} \mathrm{C}$ to $130{ }^{\circ} \mathrm{C}$, shown in Figure 3.16. The program was written to hold the sample at $20{ }^{\circ} \mathrm{C}$ for 2 minutes and increase the temperature by $5{ }^{\circ} \mathrm{C}$ per minute until the sample reached $130{ }^{\circ} \mathrm{C}$ and the sample was maintained at $130{ }^{\circ} \mathrm{C}$ for 30 minutes. In DSC measurements, the phase transformation of the sample is indicated by a narrow peak at the temperature at which the phase change occurs. From Figure 3.16 we can observe that there are no obvious peaks in the 


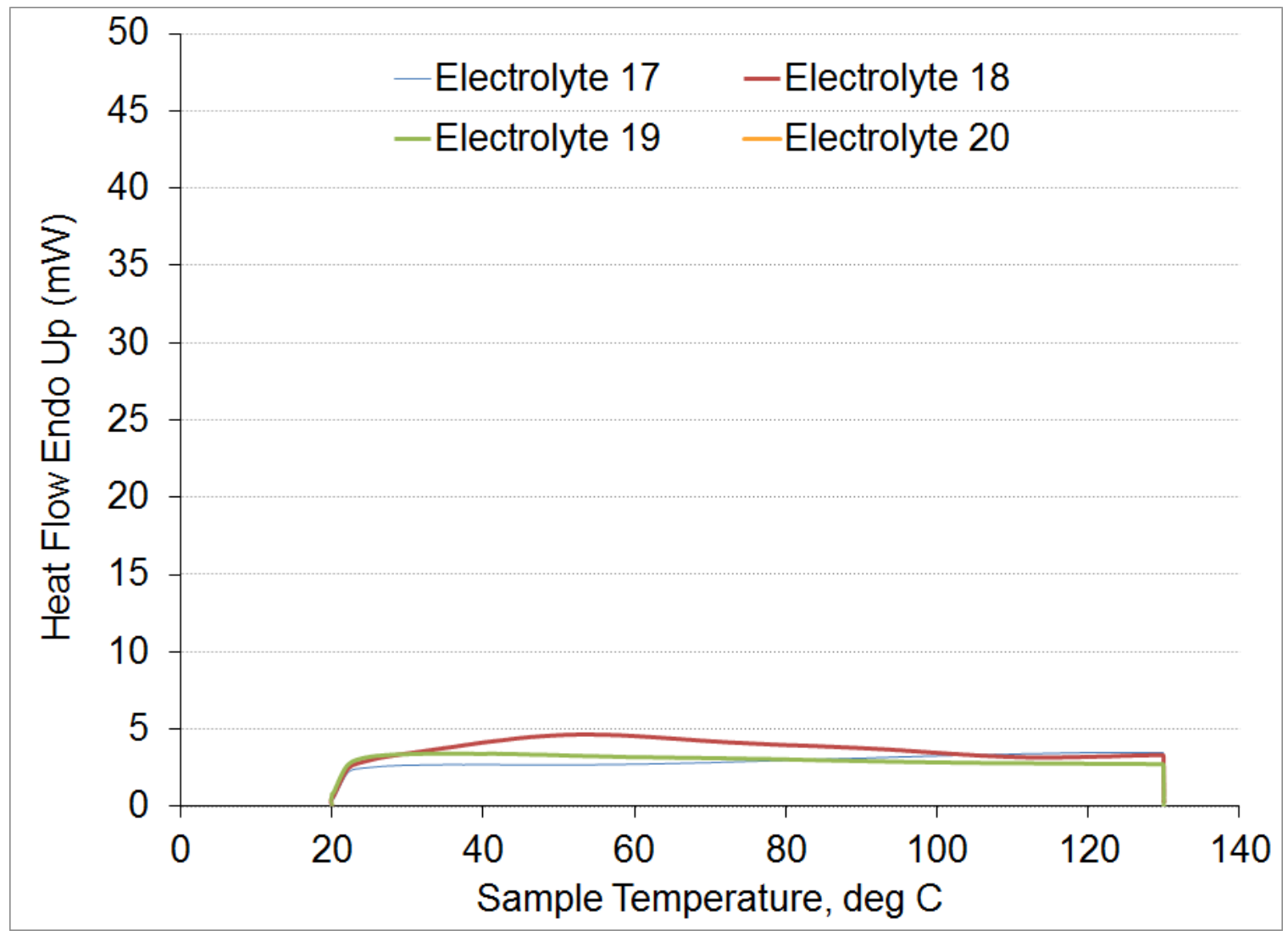

Figure 3.16: DSC measurements of electrolytes 17 to 20 from $20{ }^{\circ} \mathrm{C}$ to $130{ }^{\circ} \mathrm{C}$.

operating temperature range.

From the DSC measurements we can understand that the UV curable agents provide high stability to the electrolytes since the graphs of all the electrolytes are almost parallel to the $x$-axis. As the concentration of the UV curable agents increases the thermal stability of the electrolyte also increases, as can be observed from the graphs of electrolytes 17 and 19, they are almost perfectly parallel to the horizontal axis.

These results provide us the evidence that the presence of crosslinking agent does not affect the ionic conductivity of the electrolyte and increases the thermal stability of the electrolyte through crosslinking. 
Chapter 4

\section{DEVELOPMENT OF HEAT RESISTANT ECW}

ECWs are developed by electropolymerization PRODOT- $\mathrm{Me}_{2}$ monomer as a working electrode, $\mathrm{V}_{2} \mathrm{O}_{5}-\mathrm{TiO}_{2}$ composite materials as a counter electrode and the developed electrolytes were sandwiched between the working and the counter electrode. The ECW was now sealed using high temperature resistant epoxy adhesive and allowed to cure for 24 hours before use.

The ECW should have reasonable optical contrast before and after the heat treatment. The transmittance change of the ECW was measured using the time course measurement technique in JASCO spectrophotometer at a wavelength of $580 \mathrm{~nm}$. From the spectroscopy measurements we can conclude that the ECW has the highest contrast ratio at $580 \mathrm{~nm}$. Therefore, the time course measurements are performed at $580 \mathrm{~nm}$.

The ECWs were made and tested at room temperature first. Heat treatment testing of ECW was carried out by exposing the ECW under $80^{\circ} \mathrm{C}$ for 4 days using environment chamber. The ECW was also tested at $120^{\circ} \mathrm{C}$ for 30 minutes since the ECWs are to be laminated before selling. The ECWs should be capable of withstanding high temperature of $120^{\circ} \mathrm{C}$ for 30 minutes.

For the first two electrolytes containing lithium triflate as the lithium salt, the ECW did not show any measurable color change. This may be attributed to the bulky size of the anion, $\mathrm{CF}_{3} \mathrm{SO}_{4}^{-}$of the lithium salt and which may not intercalate completely with PPRODOT-Me $\mathrm{M}_{2}$ film . From Figure 2.3 we can see that when the anions intercalate with the EC polymer the EC polymer gets oxidized. The oxidation of PPRODOT-Me $\mathrm{Me}_{2}$ film leads to the formation of light blue transparent film. But while developing the ECW it could be observed that once the film is in the opaque state, that is intercalated with lithium ions the film does not turn transparent. This 
can be due to the lack of intercalation of $\mathrm{CF}_{3} \mathrm{SO}_{4}^{-}$anions into the PPRODOT-Me 2 film.

To resolve this problem, $\mathrm{LiClO}_{4}$ with smaller perchlorate anion, was introduced. In ECWs with electrolytes containing $\mathrm{LiClO}_{4}$ as the lithium salt, there was a visible change in optical contrast. Though epoxide-PEI matrix is unfavorable for ion movement the small size of the cation and anion allows relatively free movement. The intercalation of $\mathrm{Li}^{+}$on PProDOT-Me ${ }_{2}$ makes the electrochromic material dark blue and de-intercalation of $\mathrm{Li}^{+}$gives it light blue color. Therefore, movement of ions in the electrolyte has an important contribution towards electrochromism.

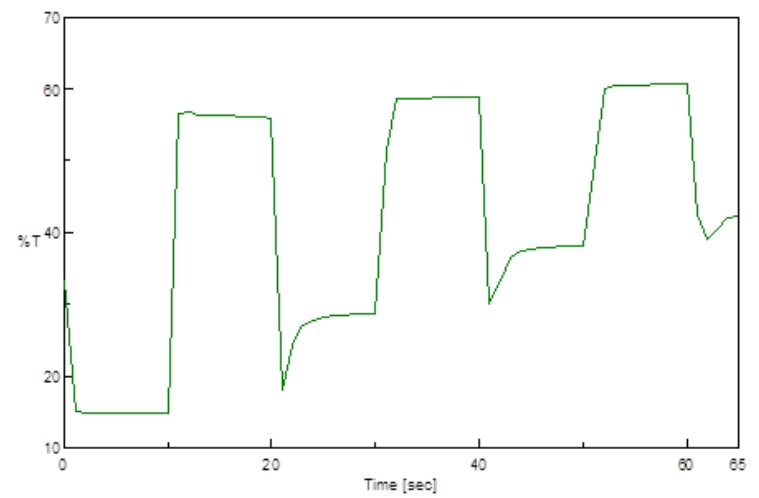

(a)

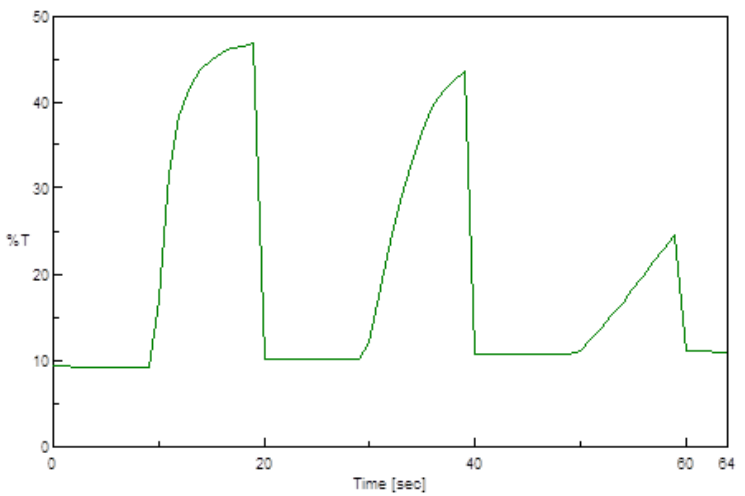

(b)

Figure 4.1: Time course measurement of ECWs with electrolytes 3, 4 and 5 . These electrolytes have lower PEI/DMSO weight ratio.

The lower the PEI/DMSO weight ratio the lower is the viscosity of the electrolyte. In the electrolyte system PEI acts as the gelation agent and swells in DMSO. Therefore, when DMSO is added to the electrolyte the viscosity of the electrolyte lowers down. When the low viscosity electrolytes are sandwiched between the working and counter electrodes in an ECW they have a tendency to flow out or move away from the center of the ECW. This can be observed from Figure 4.1 where the contrast between the opaque and the transparent states is high in the first cycle but drastically 
decreases in the second and third cycles. This can be attributed to the movement of electrolyte away from the center. Since there is minimal electrolyte present in the center the color switching of the PPRODOT-Me $\mathrm{Me}_{2}$ film is negligible.

The problem can be resolved if the viscosity of the electrolyte is increased, and this can be achieved in the following three ways,

1. Decrease the concentration of the DMSO responsible for lowering the viscosity of the electrolyte.

2. Introduce crosslinking agents that react with amine group to form gel like electrolyte.

3. Use UV curable agents to fix the electrolyte in place.

In electrolyte no 6,7 and 8 the concentration of the DMSO was decreased and $\mathrm{LiClO}_{4}$ to increase the viscosity of the electrolyte and to modulate the number of free lithium ions present as free ions since higher the concentration of lithium salt lower is the concentration of free lithium ions due to the formation of contact ion pairs or triple ions.

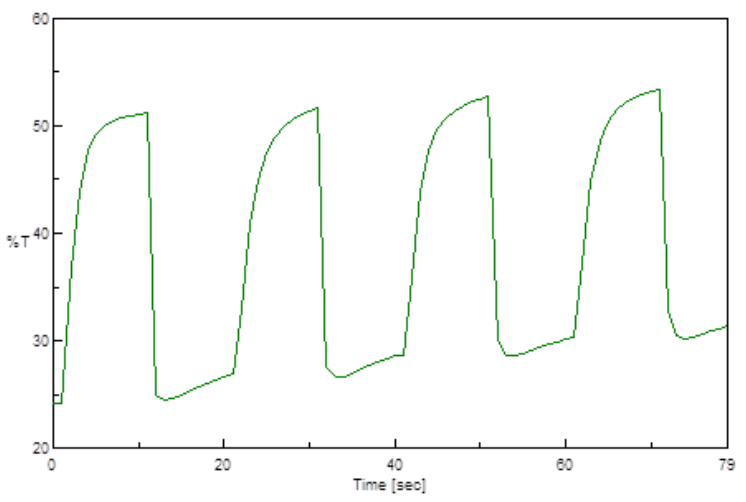

(a)

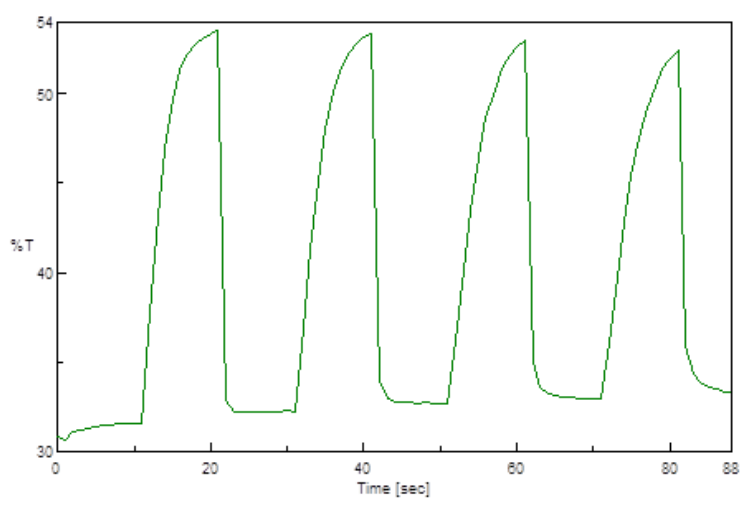

(b)

Figure 4.2: Time course measurement of ECWs with electrolytes 8, (a) before the heat treatment, (b) after the heat treatment. 
Electrolytes no 10 to 16 have different concentrations of the crosslinking agent DGEBA as mentioned in Chapter 3. The different concentrations of DGEBA were tried to test the change in transmittance of the electrolytes with temperature and the compatibility of the electrolytes with the working and counter electrode materials, shown in Figure 4.3.

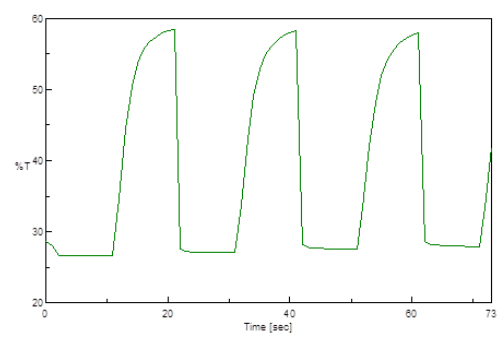

(a)

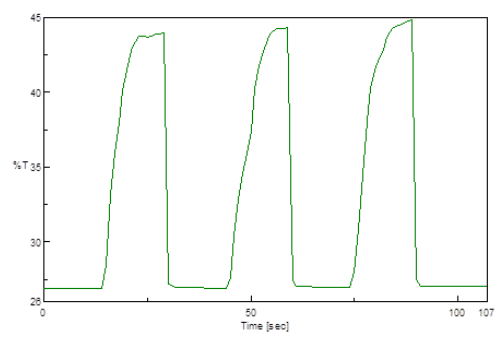

(b)

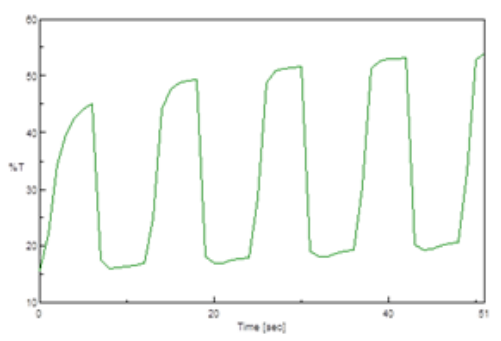

(c)

Figure 4.3: Time course measurement of ECWs with electrolyte 10 (a) Before the heat treatment, (b) after the heat treatment at $80^{\circ} \mathrm{C}$ for 100 hours, (c) after the heat treatment at $120^{\circ} \mathrm{C}$ for 30 minutes.

Figure 4.4, shows the optical transmittance of ECW with electrolyte no 11. The electrolyte no 11 also shows reasonable contrast ratio and stability. But electrolyte no 10 shows better stability over time. Therefore, optimized electrolyte composition must lie between electrolyte no 10 and 11.

The third solution of introducing UV curable agents into the electrolyte was implemented in electrolyte no 17 to electrolyte no 20. The performance of the electrolyte with ECW was tested. Figure 36, shows the performance of the ECW before and after the heat treatment. The ECW shows reasonable stability before and after heat treatment. This shows that the electrolyte does not move away from the center even at higher temperature. 


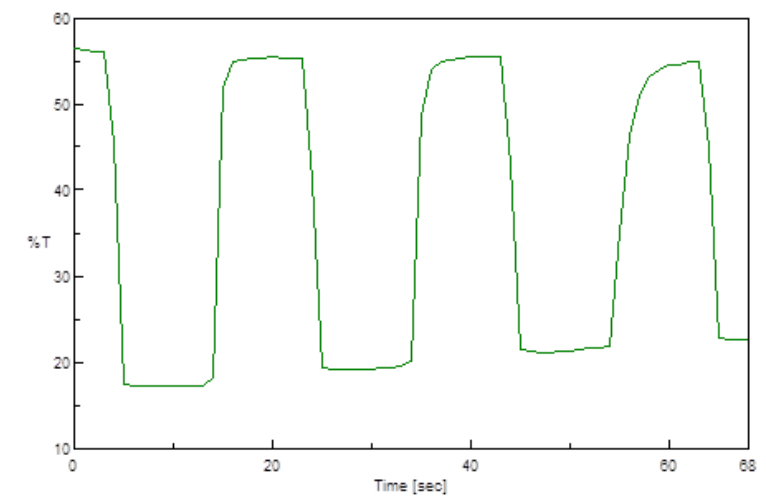

(a)

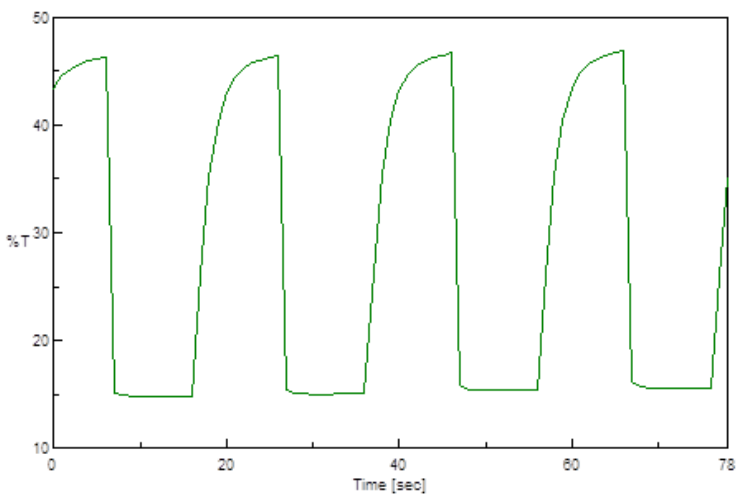

(b)

Figure 4.4: Time course measurement of ECWs with electrolyte 11 (a) Before the heat treatment, (b) after the heat treatment.

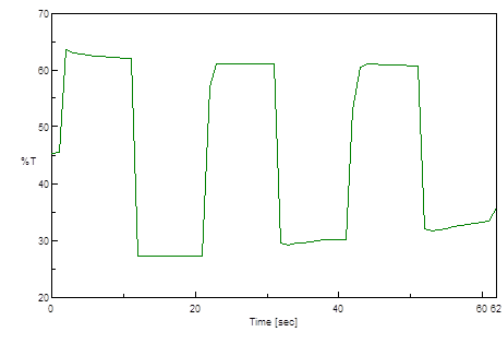

(a)

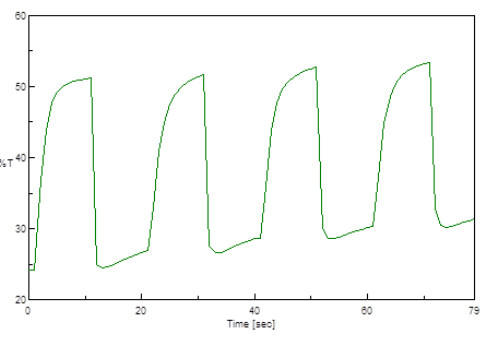

(b)

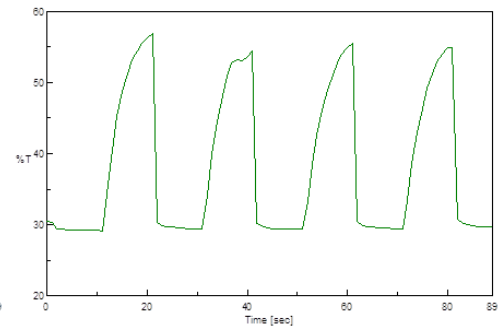

(c)

Figure 4.5: Time course measurement of ECWs with electrolyte 18 (a) Before the heat treatment, (b) after the heat treatment at $80^{\circ} \mathrm{C}$ for 100 hours, (c) after the heat treatment at $120^{\circ} \mathrm{C}$ for 30 minutes. 


\section{Chapter 5}

\section{CONCLUSION}

High temperature resistant ECW for the application of sunroof in automobiles has been developed. The developed poly(ethylene imine) based electrolyte has high transmittance and ionic conductivity before and heat treatment upto $120^{\circ} \mathrm{C}$. In the case of PEI based electrolytes, electrolyte no 8 with $\mathrm{PEI} / \mathrm{DMSO} / \mathrm{LiClO}_{4}$ weight ratio of 50/55/1.50 gives the best data. The electrolyte maintains a high transmittance of $70 \%$ at all range of temperatures and also maintains high ionic conductivity and chemical stability. The ECW with electrolyte no 8 exhibits a transmittance of $52 \%$ in transparent state and $24 \%$ in opaque state. This changes to $52 \%$ and $33 \%$, respectively after the heat treatment at $80^{\circ} \mathrm{C}$ for 100 hours.In the next case of PEI-DGEBA based electrolytes, electrolyte no 10 with $\mathrm{PEI} / \mathrm{DMSO} / \mathrm{DGEBA} / \mathrm{LiClO} 4$ weight ratio of 50/44/0.8/1.50 gives the best data. The electrolyte maintains a high transmittance of $67 \%$ at all range of temperatures and also maintains high ionic conductivity and chemical stability. The ECW with electrolyte no 10 exhibits a transmittance of $57 \%$ in transparent state and $27 \%$ in opaque state. This changes to $45 \%$ and $22 \%$, respectively, after the heat treatment at $80^{\circ} \mathrm{C}$ for 100 hours. The transmittance changes to $45 \%$ and $23 \%$ after heat treatment at $120^{\circ} \mathrm{C}$ for 30 minutes. In the last case of UV curable electrolytes, electrolyte no 18 is the most promising electrolyte composition. The ECW shows a maximum transmittance of $63 \%$ and minimum of $27 \%$. After the heat treatment at $80^{\circ} \mathrm{C}$ for 100 hours, the maximum transmittance drops to $50 \%$ and $24 \%$ respectively. The maximum transmittance reduces to $55 \%$ and minimum to $30 \%$ after heat treatment at $120^{\circ} \mathrm{C}$ for 30 minutes.

The maximum transmittance of the ECW should be improved compared to the current achievable value to effectively apply the ECW for sunroof applications. This can be achieved by further optimizing the concentration of crosslinking agents be- 
tween electrolyte 10 and 11. To further increase the thermal stability of the electrolyte, ionic gel electrolytes may be considered. Ionic gels are molten salts dispersed in suitable polymer matrix. These electrolytes are proven to be thermally stable over a wide range of temperatures. The compatibility of these electrolytes with the working and counter electrode material should be tested and the composition should be optimized. 


\section{BIBLIOGRAPHY}

[1] World Business Council for Sustainable Development, 2009. Energy Performance in Buildings: Transforming the Market, IS 200965.

[2] D. Arasteh, S. Selkowitz, J. Apte, and M. LaFrance, Zero Energy Windows, Lawrence Berkeley Natl. Lab., May 2006.

[3] Handbook of Inorganic Electrochromic Materials, edited by C.G. Granqvist

[4] B.W. Faughnan, R.S. Crandall, P.M. Heyman, Electrochromism in $\mathrm{WO}_{3}$ amorphous films, RCA Review, 36 (1975), pp. 177197

[5] S.F. Cogan, N.M. Nguyen, S.J. Perrotti, R.D. Rauh, Optical properties of electrochromic vanadium pentaoxide, Journal of Applied Physics, 66 (1989), pp. 13331337

[6] D.M. DeLongchamp, P.T. Hammond, High-contrast electrochromism and controllable dissolution of assembled Prussian blue/polymer nanocomposites, Advanced Functional Materials, 14 (2004), pp. 224232

[7] L. Cao, Y. Wang, Preparation and mechanism of electrochromic viologens, Progress of Chemistry, 20 (2008), pp. 13531360

[8] F. Rourke, J.A. Crayston, Cyclic voltammetry and morphology of polyaniline-coated electrodes containing Fe(CN)63/4 ions, Journal of the Chemical Society, Faraday Transactions, 89 (1993), pp. 295302

[9] C.M. Amb, A.L. Dyer, J.R. Reynolds, Navigating the color palette of solutionprocessable electrochromic polymers, Chemistry of Materials, 23 (2011), pp. 397415

[10] P.R. Somani, S. Radhakrishnan, Electrochromic materials and devices: present and future, Materials Chemistry and Physics, 77 (2003), pp. 117133

[11] Y. Yan, J. Huang, Hierarchical assemblies of coordination supramolecules, Coordination Chemistry Reviews, 254 (2010), pp. 10721080

[12] D.M. Roundhill, Photochemistry and Photophysics of Metal Complexes, Plenum, New York (1994) 
[13] S. Salmaoui, F. Sediri, N. Gharbi, C. Perruchot, M. Jouini, Hexagonal hydrated tungsten oxide nanomaterials: Hydrothermal synthesis and electrochemical properties, Electrochim. Acta, 108 (2013), p. 634

[14] X. Chang, S. Sun, L. Dong, X. Hu, Y. Yin Tungstun oxide nanowires grown on graphene oxide sheets as high performance electrochromic material, Electrochimica acta 129(2014) 40-46

[15] J. Wang, E. Khoo, P.S. Lee, J. Ma, Controlled synthesis of WO3 nanorods and their electrochromic properties in H2SO4 electrolyte, J. Phys. Chem. C, 113 (2009), p. 9655

[16] J. Zhang, J.P. Tu, X.H. Xia, X.L. Wang, C.D. Gu, Hydrothermally synthesized $\mathrm{WO}_{3}$ nanowire arrays with highly improved electrochromic performance, J. Mater. Chem., 21 (2011), p. 5492

[17] C.K. Wang, C.K. Lin, C.L. Wu, S.C. Wang, J.L. Huang, Synthesis and characterization of electrochromic plate-like tungsten oxide films by acidic treatment of electrochemical anodized tungsten, Electrochim. Acta, 112 (2013), pp. 2431

[18] A.K. Geim, Graphene: status and prospects, Science, 324 (2009), p. 1530

[19] Y.W. Zhu, S. Murali, W.W. Cai, X.S. Li, J.W. Suk, J.R. Potts, R.S. Ruoff, Graphene and graphene oxide: synthesis, properties, and applications, Adv. Mater., 22 (2010), p. 3906

[20] Q.J. Xiang, J.G. Yu, M. Jaroniec, Graphene-based semiconductor photocatalysts, Chem. Soc. Rev., 41 (2012), p. 782

[21] Y.Y. Liang, Y.G. Li, H.L. Wang, J.G. Zhou, J. Wang, T. Regier, H.J. Dai, $\mathrm{Co}_{3} \mathrm{O}_{4}$ nanocrystals on graphene as a synergistic catalyst for oxygen reduction reaction, Nature Mater., 10 (2011), p. 780

[22] J. Yan, T. Wei, W. Qiao, B. Shao, Q. Zhao, L. Zhang, Z. Fan, Rapid microwave-assisted synthesis of graphene nanosheet/Co3O4 composite for supercapacitors, Electrochim. Acta, 55 (2010), p. 6973

[23] H.L. Wang, L.F. Cui, Y.A. Yang, H.S. Casalongue, J.T. Robinson, Y.Y. Liang, Y. Cui, H.J. Dai, $\mathrm{Mn}_{3} \mathrm{O}_{4}$-graphene hybrid as a high-capacity anode material for lithium ion batteries, J. Am. Chem. Soc., 132 (2010), p. 13978

[24] Y.W. Zhu, S. Murali, M.D. Stoller, K.J. Ganesh, W.W. Cai, P.J. Ferreira, A. Pirkle, R.M. Wallace, K.A. Cychosz, M. Thommes, D. Su, E.A. Stach, R.S. Ruoff, Carbonbased supercapacitors produced by activation of graphene, Science, 332 (2011), p. 1537 
[25] H. Wang, J.T. Robinson, X. Li, H. Dai, Solvothermal reduction of chemically exfoliated graphene sheets, J. Am. Chem. Soc., 131 (2009), p. 9910

[26] A.B. Kaiser, C. Gomez-Navarro, R.S. Sundaram, M. Burghard, K. Kern, Electrical conduction mechanism in chemically derived graphene monolayers, Nano Lett., 9 (2009), p. 178

[27] R. J. Mortimer, Electrochromic materials, Chem. Soc. Rev., vol. 26, no. 3, p. 147, Jan. 1997.

[28] R. J. Mortimer, A. L. Dyer, and J. R. Reynolds, Electrochromic organic and polymeric materials for display applications, Displays, vol. 27, no. 1, pp. 218, Jan. 2006.

[29] M. Li, Y. Wei, J. Zheng, D. Zhu, and C. Xu, Highly contrasted and stable electrochromic device based on well-matched viologen and triphenylamine, Org. Electron., vol. 15, no. 2, pp. 428434, Feb. 2014.

[30] K. Choi, S. J. Yoo, Y.-E. Sung, and R. Zentel, High Contrast Ratio and Rapid Switching Organic Polymeric Electrochromic Thin Films Based on Triarylamine Derivatives from Layer-by-Layer Assembly, Chem. Mater., vol. 18, no. 25, pp. 58235825, Dec. 2006.

[31] R. J. Mortimer, Organic electrochromic materials, Electrochim. Acta, vol. 44, no. 18, pp. 29712981, May 1999.

[32] R. G. Compton, A. M. Waller, P. M. S. Monk, and D. R. Rosseinsky, Electron paramagnetic resonance spectroscopy of electrodeposited species from solutions of 1,1?-bis-(pcyanophenyl)-4,4?-bipyridilium (cyanophenyl paraquat, CPQ), J. Chem. Soc. Faraday Trans., vol. 86, no. 14, p. 2583, Jan. 1990.

[33] E. Steckhan, Ed., Electrochemistry IV, vol. 152. Berlin/Heidelberg: Springer-Verlag, 1990 .

[34] J. Roncali, Conjugated poly(thiophenes): synthesis, functionalization, and applications, Chem. Rev., vol. 92, no. 4, pp. 711738, Jun. 1992.

[35] M. Mastragostino, C. Arbizzani, A. Bongini, G. Barbarella, and M. Zambianchi, Polymer-based electrochromic devicesI. Poly(3-methylthiophenes), Electrochim. Acta, vol. 38, no. 1, pp. 135140, Jan. 1993.

[36] Roundhill, D. Max. Photochemistry and photophysics of metal complexes. New York: Plenum Press, 1994.

[37] M. Higuchi, Electrochromic OrganicMetallic Hybrid Polymers: Fundamentals and Device Applications, Polym. J., vol. 41, no. 7, pp. 511520, May 2009. 
[38] S. Bernhard, J. I. Goldsmith, K. Takada, and H. D. Abrua, Iron(II) and copper(I) coordination polymers: electrochromic materials with and without chiroptical properties., Inorg. Chem., vol. 42, no. 14, pp. 438993, Jul. 2003.

[39] Kurth, Dirk G., Markus Schtte, and Jin Wen. Metallo-supramolecular polyelectrolyte multilayers with cobalt (II): preparation and properties. Colloids and Surfaces A: Physicochemical and Engineering Aspects 198 (2002): 633-643.

[40] E. C. Constable, The Coordination Chemistry of 2,2:6,2-Terpyridine and Higher Oligopyridines, Adv. Inorg. Chem., vol. 30, no. C, pp. 69121, 1986.

[41] V. A. Friese and D. G. Kurth, From coordination complexes to coordination polymers through self-assembly, Curr. Opin. Colloid Interface Sci., vol. 14, no. 2, pp. 8193, Apr. 2009 .

[42] F. S. Han, M. Higuchi, and D. G. Kurth, Metallo-Supramolecular Polymers Based on Functionalized Bis-terpyridines as Novel Electrochromic Materials, Adv. Mater., vol. 19, no. 22, pp. 39283931, Nov. 2007.

[43] J.-H. Li and M. Higuchi, Substituent Effects on Metallo-Supramolecular Coordination Polymers, J. Inorg. Organomet. Polym. Mater., vol. 20, no. 1, pp. 1018, Feb. 2010.

[44] Selkowitz, Stephen, et al. Window systems for high-performance buildings. New York: Norton, 2004.

[45] On the Heating of Parked Cars - Doing Science To Stuff on WordPress.com. (n.d.). Retrieved May 22, 2014, from http : //blog.doingsciencetostuff.com/2013/07/18/onthe - heating - of - parked-cars/

[46] Greenhouse Effect: Background Material. (n.d.). Retrieved June 11, 2014, from http : //www.ucar.edu/learn/1_3_1.htm

[47] Amasawa, E. (2013, February 24). Design and characterization of a durable and highly efficient energy-harvesting electrochromic window. Retrieved from https : //digital.lib.washington.edu : 443/xmlui/handle/1773/25168

[48] The Polyene Series - CMDITRWIKI. (n.d.). Retrieved May 28, 2014, from http : $/ /$ photonicswiki.org/index.php?title $=$ The $_{\text {Polyene }}$ eries

[49] Xu, J., Nie, G., Zhang, S., Han, X., Hou, J., \& Pu, S. (2005). Electrochemical copolymerization of indole and 3,4-ethylenedioxythiophene. Journal of Materials Science, 40(11), 28672873. doi:10.1007/s10853-005-2421-x 
[50] Funt, B. L., \& Bhadani, S. N. (1964). ELECTROINITIATED POLYMERIZATION OF STYRENE: II. KINETICS IN SOLUTIONS OF TETRAMETHYLAMMONIUM SALTS. Canadian Journal of Chemistry, 42(12), 27332738. doi:10.1139/v64-404

[51] Inzelt, G. (2012). Conducting Polymers. Berlin, Heidelberg: Springer Berlin Heidelberg. doi:10.1007/978-3-642-27621-7

[52] Kim, Sooyeun, and Minoru Taya. "Electropolymerization kinetic study of 3, 3-dimethyl3, 4-dihydro-2H-thieno [3, 4-b][1,4] dioxepine and its optical optimization for electrochromic window applications." Electrochimica Acta 55.19 (2010): 5307-5311.

[53] Dkhissi, A., Louwet, F., Groenendaal, L., Beljonne, D., Lazzaroni, R., \& Brdas, J. L. (2002). Theoretical investigation of the nature of the ground state in the lowbandgap conjugated polymer, poly(3,4-ethylenedioxythiophene). Chemical Physics Letters, 359(5-6), 466472. doi:10.1016/S0009-2614(02)00651-6

[54] Takahashi, K., Limmer, S. J., Wang, Y., \& Cao, G. (2004). Synthesis and Electrochemical Properties of Single-Crystal $\mathrm{V}_{2} \mathrm{O}_{5}$ Nanorod Arrays by Template-Based Electrodeposition. The Journal of Physical Chemistry B, 108(28), 97959800. doi:10.1021/jp0491820

[55] Ohno, Hiroyuki, ed. Electrochemical aspects of ionic liquids. John Wiley \& Sons, 2011.

[56] Chiang, C., Davis, G., Harding, C., \& Takahashi, T. (1986). Polymeric electrolyte based on poly(ethylene imine) and lithium salts. Solid State Ionics, 18-19, 300305. doi:10.1016/0167-2738(86)90131-1

[57] Shriver, D. F., Papke, B. L., Ratner, M. A., Dupon, R., Wong, T., \& Brodwin, M. (1981). Structure and ion transport in polymer-salt complexes. Solid State Ionics, 5(C), 8388. Retrieved from http://www.scopus.com/inward/record.url?eid $=2-s 2.0-$ 0019623862\&partnerID $=t Z O t x 3 y 1$

[58] Unal, B., Klein, R. J., Yocca, K. R., \& Hedden, R. C. (2007). Influence of DGEBA crosslinking on $\mathrm{Li}^{+}$ion conduction in poly(ethyleneimine) gels. Polymer, 48(20), 60776085. doi:10.1016/j.polymer.2007.08.003

[59] Campet, G., et al. "PLR (Plastic Lithium Rechargeable) Batteries using Nanoscale Materials: A Convenient Source of Electrical Energy for the Future?." BULLETINKOREAN CHEMICAL SOCIETY 20 (1999): 885-892.

[60] Song, J. Y., Wang, Y. Y., \& Wan, C. C. (1999). Review of gel-type polymer electrolytes for lithium-ion batteries. Journal of Power Sources, 77(2), 183197. doi:10.1016/S03787753(98)00193-1 
[61] Snow, A. G., Sanders, R. A., Frech, R., \& Glatzhofer, D. T. (2003). Synthesis and spectroscopic studies of linear poly(N-(2-(2-methoxyethoxy)ethyl)ethylenimine), a PEI/PEO hybrid, and its interactions with lithium triflate. Electrochimica Acta, 48(14-16), 20652069. doi:10.1016/S0013-4686(03)00186-5

[62] IONESCUVASII, L., GARCIA, B., \& ARMAND, M. (2006). Conductivities of electrolytes based on PEI-b-PEO-b-PEI triblock copolymers with lithium and copper TFSI salts. Solid State Ionics, 177(9-10), 885892. doi:10.1016/j.ssi.2006.01.024

[63] IONESCUVASII, L., ABULEBDEH, Y., \& ARMAND, M. (2005). Synthesis and characterization of new block copolymer electrolytes with solvating affinities for different cations. Solid State Ionics, 176(37-38), 27692775. doi:10.1016/j.ssi.2005.08.014

[64] Dielectric Constant. (n.d.). Retrieved June 01, 2014, from http : //macro.lsu.edu/howto/solvents/DielectricConstant.htm

[65] Coordinate Covalent Bonding - EasyChem - The Ultimate Resource for HSC Chemistry: Syllabus-Based Dot-Point Study Notes/Summaries, Past Exam Papers, and More. (n.d.). Retrieved June 02, 2014, from http : //www.easychem.com.au/monitoring - and - management/the atmosphere/coordinate - covalent - bonding

[66] Bisphenol A diglycidyl ether — Sigma-Aldrich. (n.d.). Retrieved June 12, 2014, from http://www.sigmaaldrich.com/catalog/product/sigma/d3415?lang $=$ en\&region $=$ $U S$

[67] Polyethylenimine, branched average $\mathrm{Mw} 25,000$ by LS, average Mn 10,000 by GPC, branched — Sigma-Aldrich. (n.d.). Retrieved June 12, 2014, from http : //www.sigmaaldrich.com/catalog/product/aldrich/408727?lang = en\&region $=U S$

[68] Poly(ethylene glycol) methyl ether methacrylate average $\mathrm{Mn} 500$, contains $100 \mathrm{ppm}$ MEHQ as inhibitor, $200 \mathrm{ppm}$ BHT as inhibitor - Sigma-Aldrich. (n.d.). Retrieved June 02, 2014, from http : //www.sigmaaldrich.com/catalog/product/aldrich/447943?lang $=$ en\&region $=U S$

[69] Poly(ethylene glycol) average mol wt 200 - Sigma-Aldrich. (n.d.). Retrieved June 02, 2014, from http : //www.sigmaaldrich.com/catalog/product/sial/p3015?lang = en\&region $=U S$

[70] 2,2-Bis(hydroxymethyl)propionic acid 98\% — Sigma-Aldrich. (n.d.). Retrieved June 12, 2014, from http ://www.sigmaaldrich.com/catalog/product/aldrich/106615?lang = en\&region $=U S$ 
Appendix A

\section{EXPERIMENTAL RESULTS: VARIATION OF TRANSMITTANCE OF ELECTROLYTES FROM $20^{\circ} \mathrm{C}$ TO $120^{\circ} \mathrm{C}$}
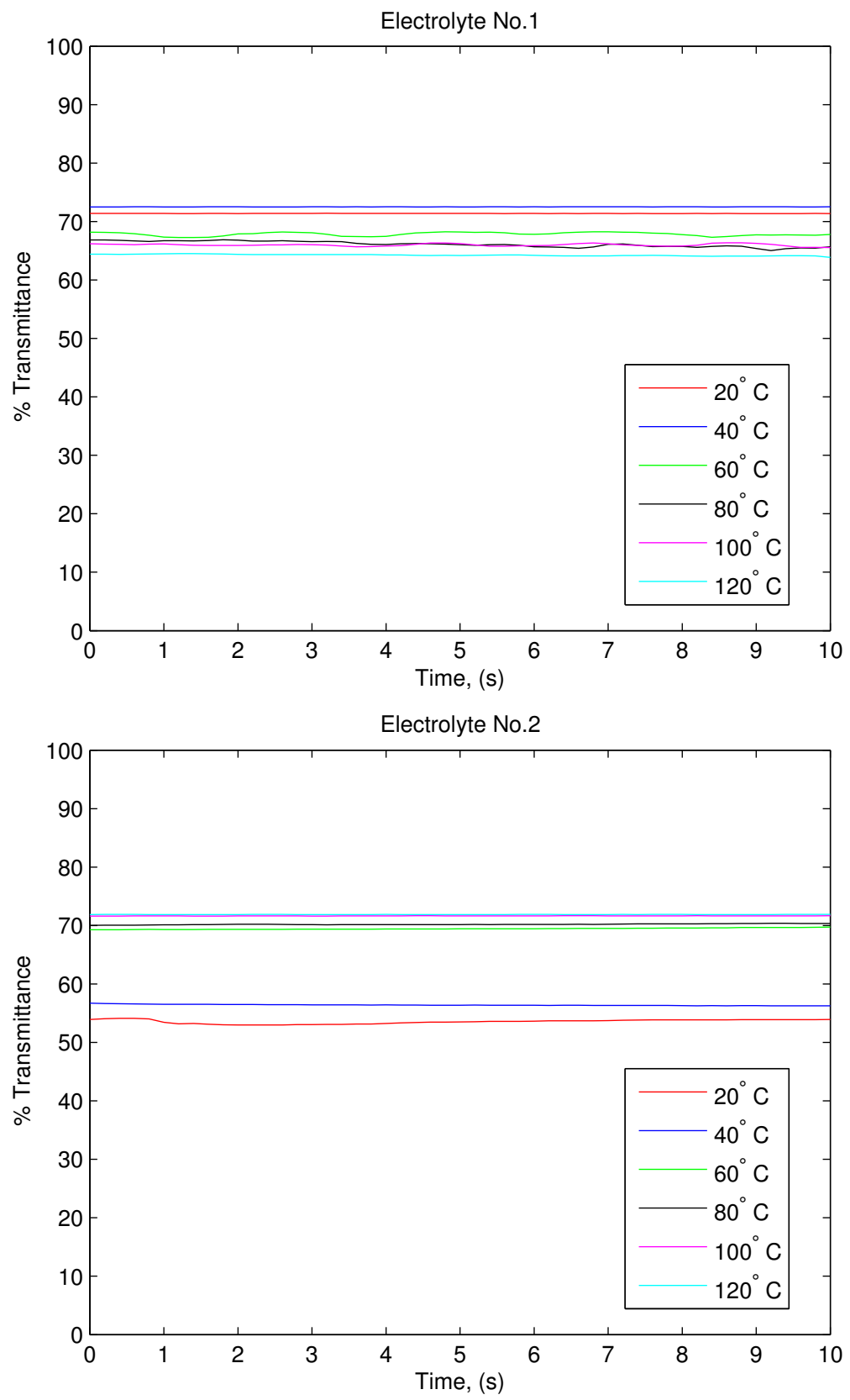

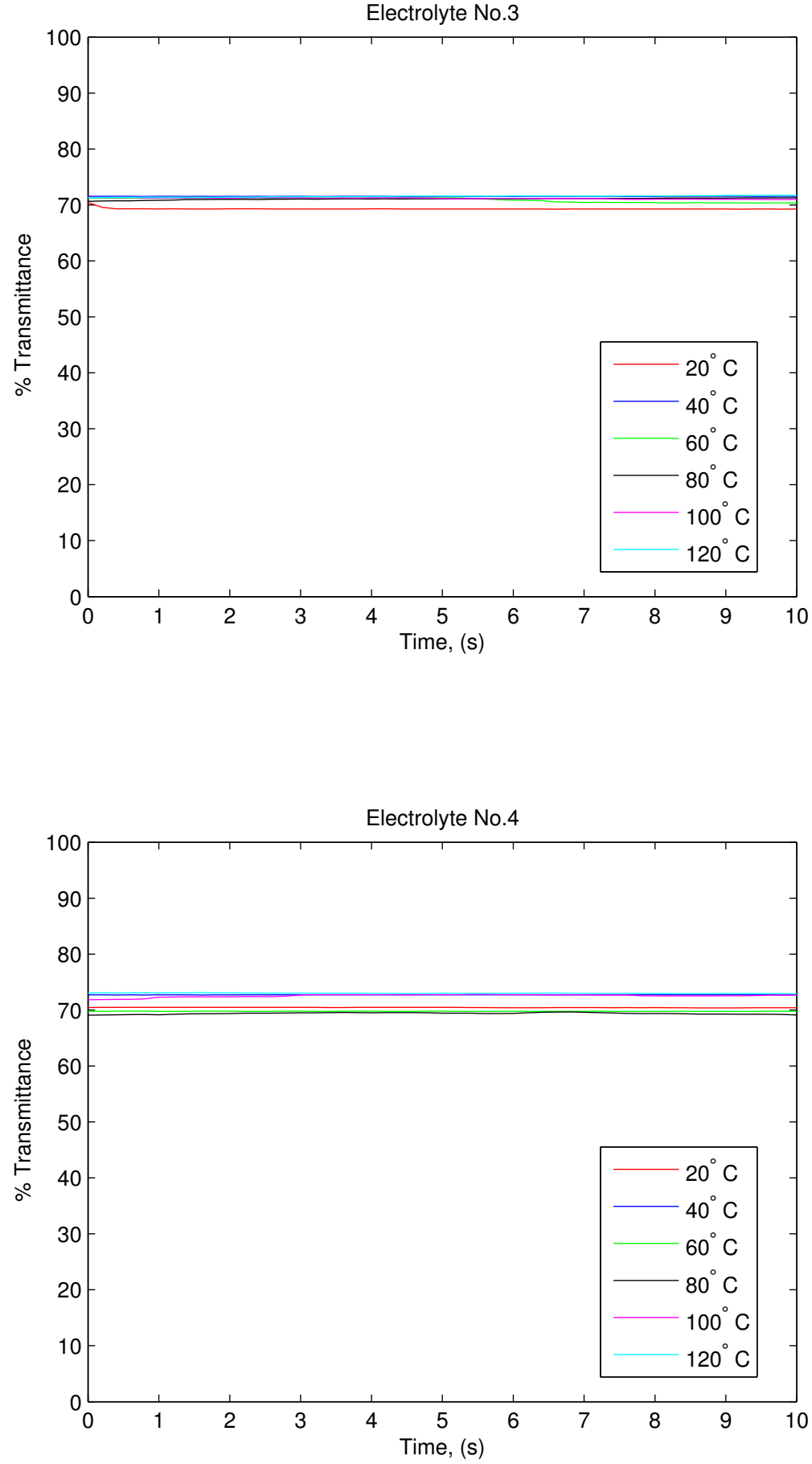
Electrolyte No.5
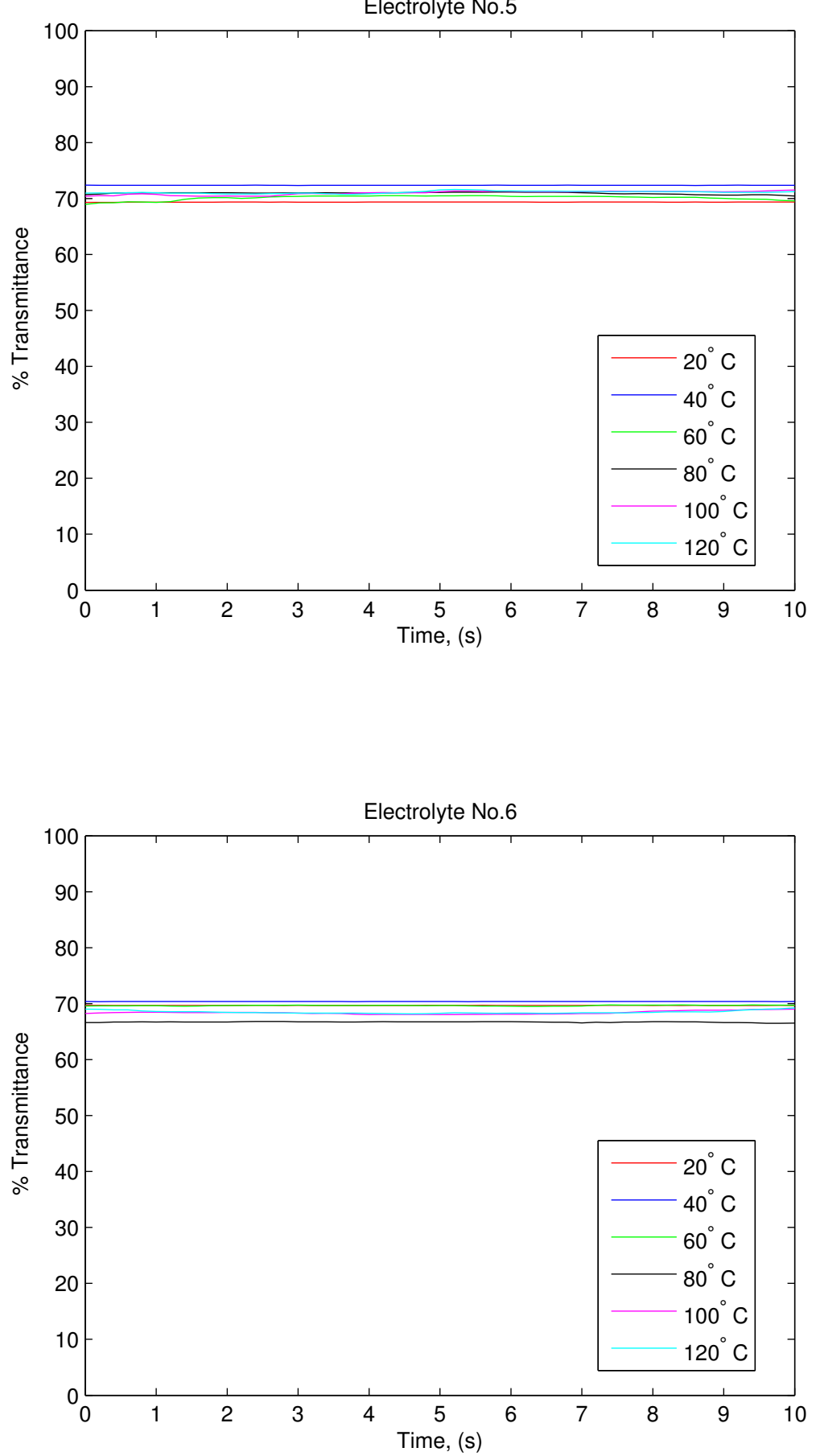

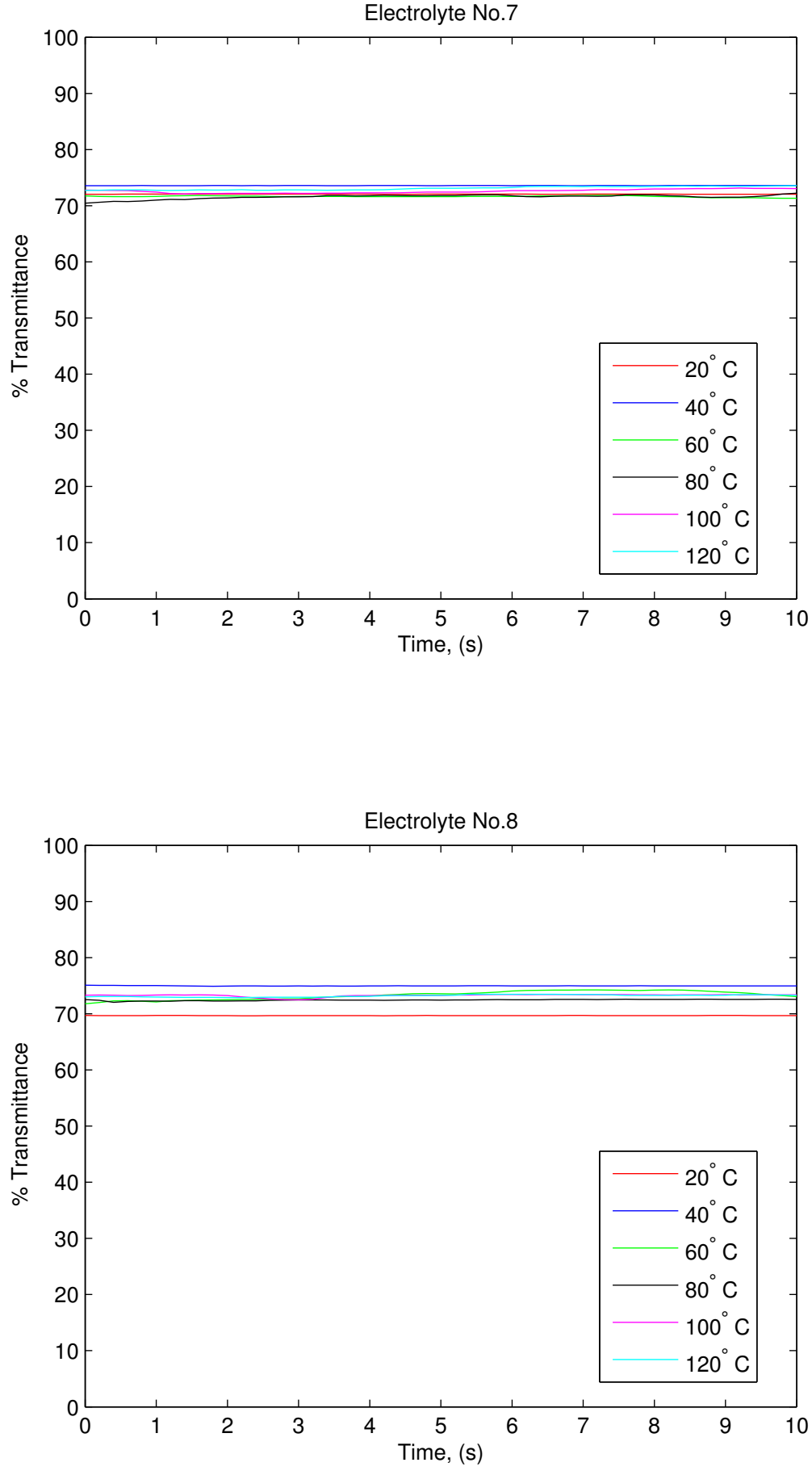
Electrolyte No.9
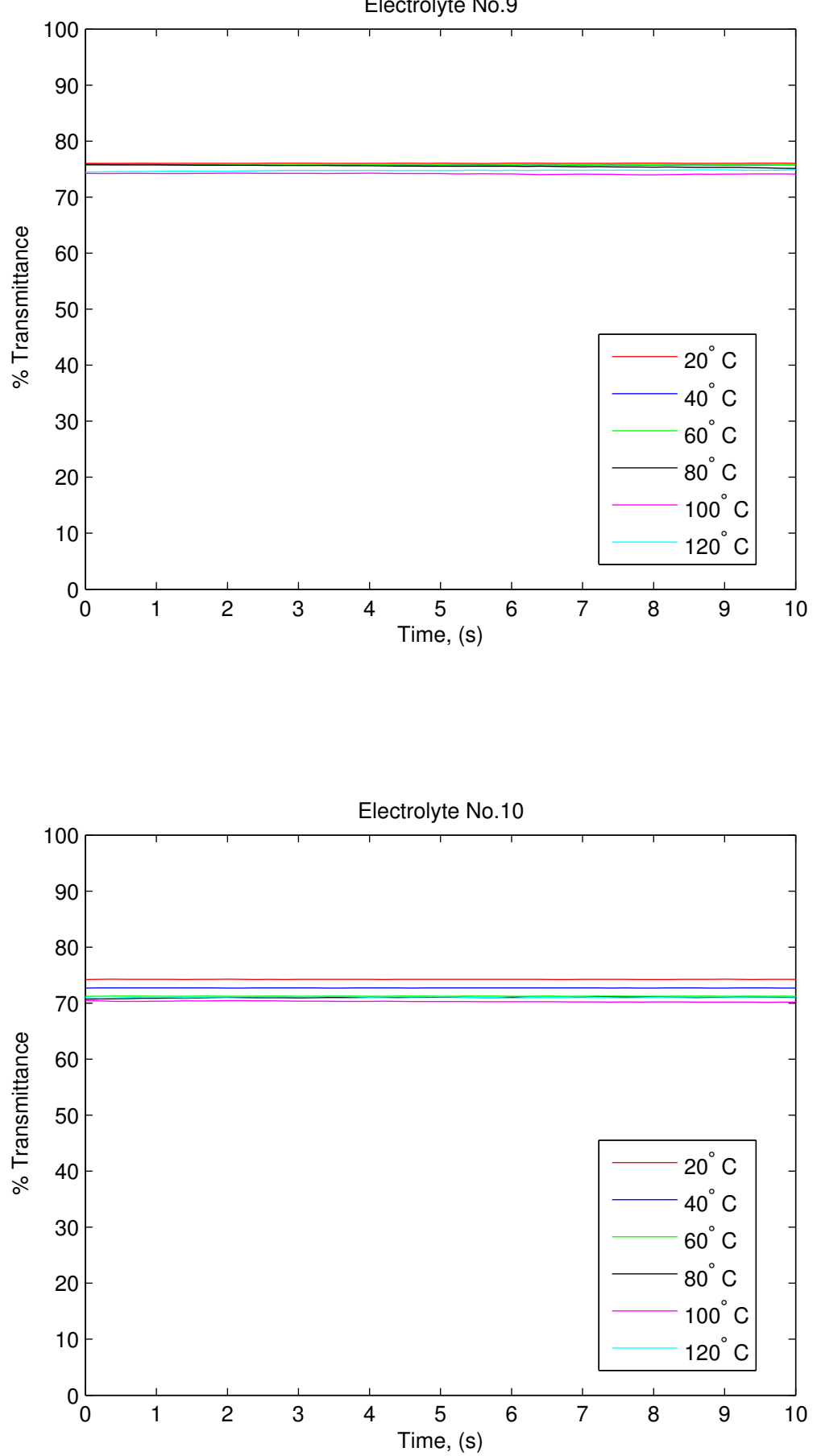

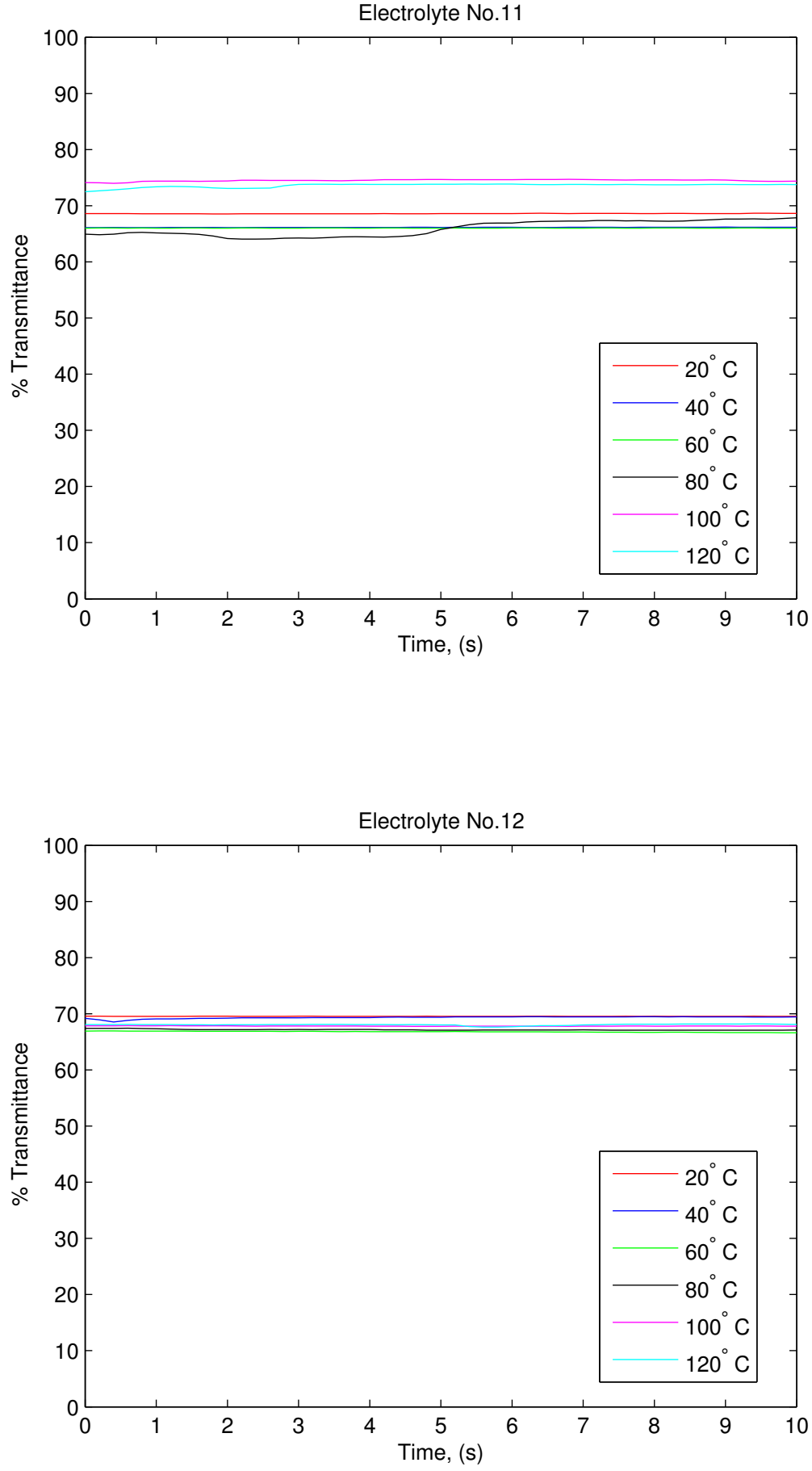

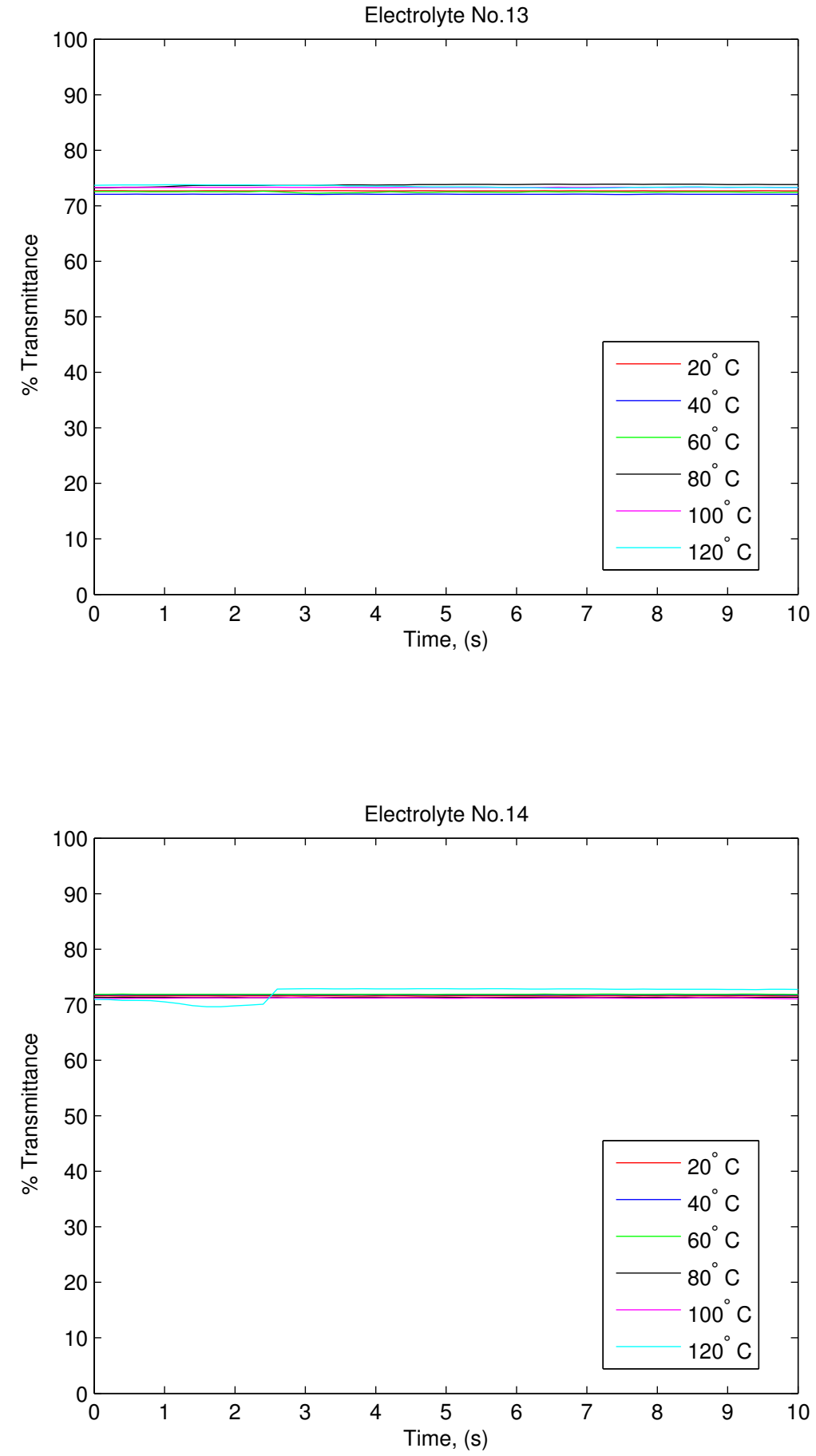
Electrolyte No.15

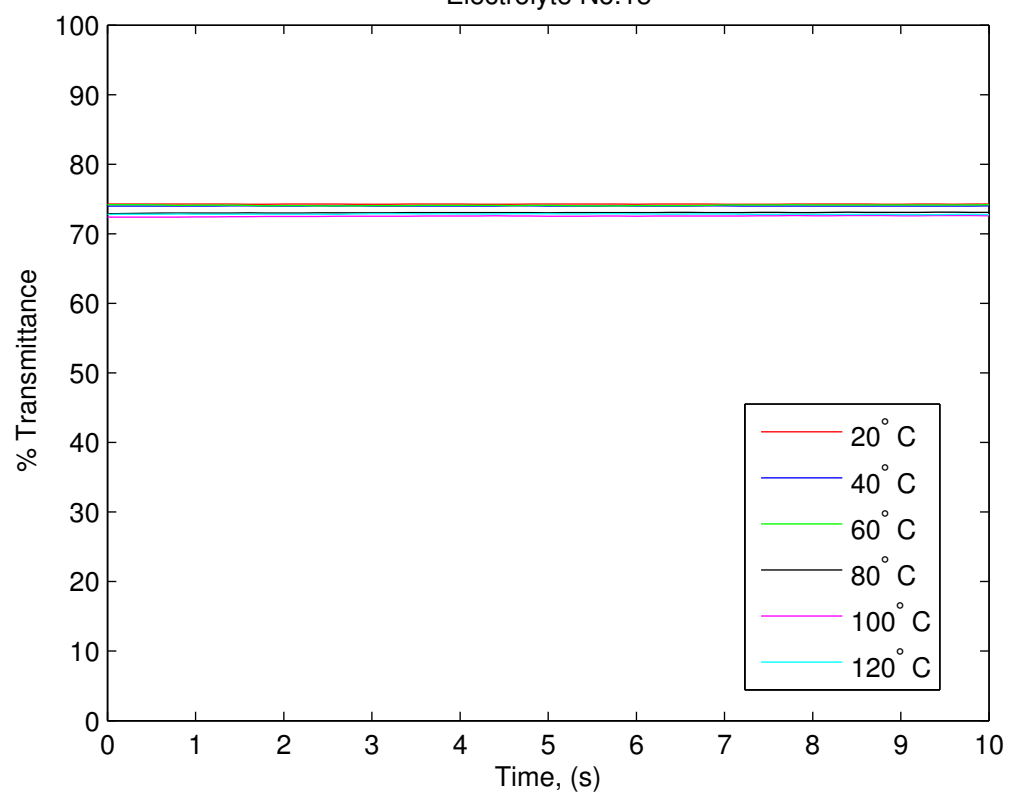

Electrolyte No.16

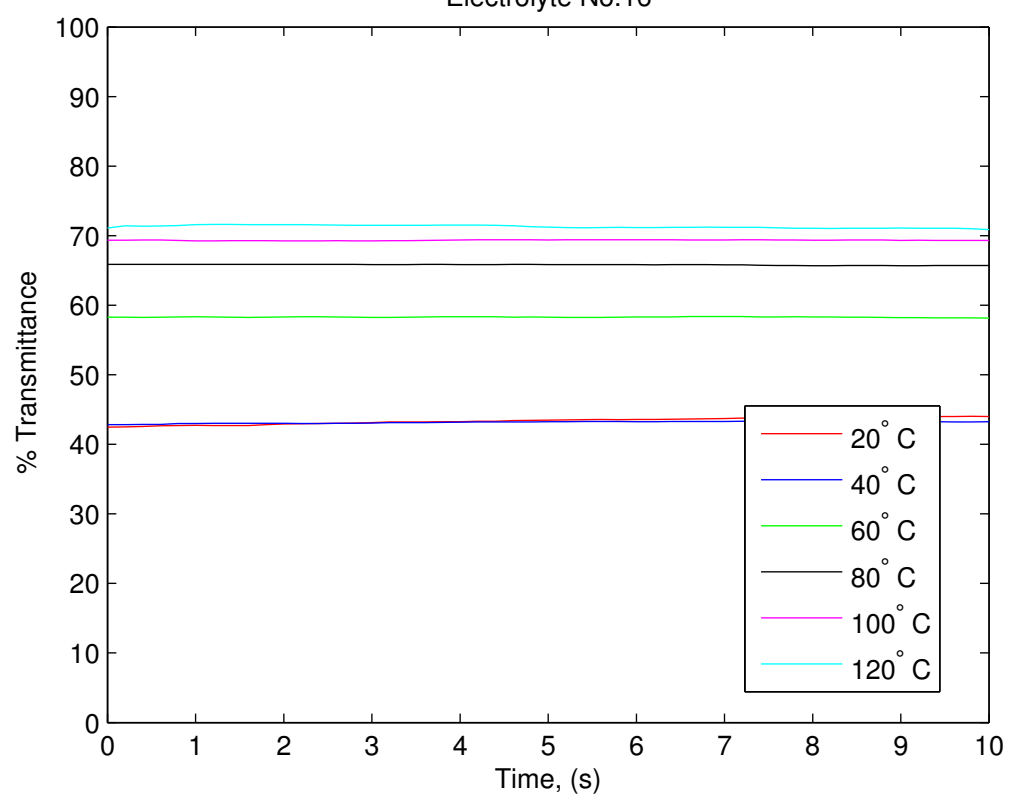



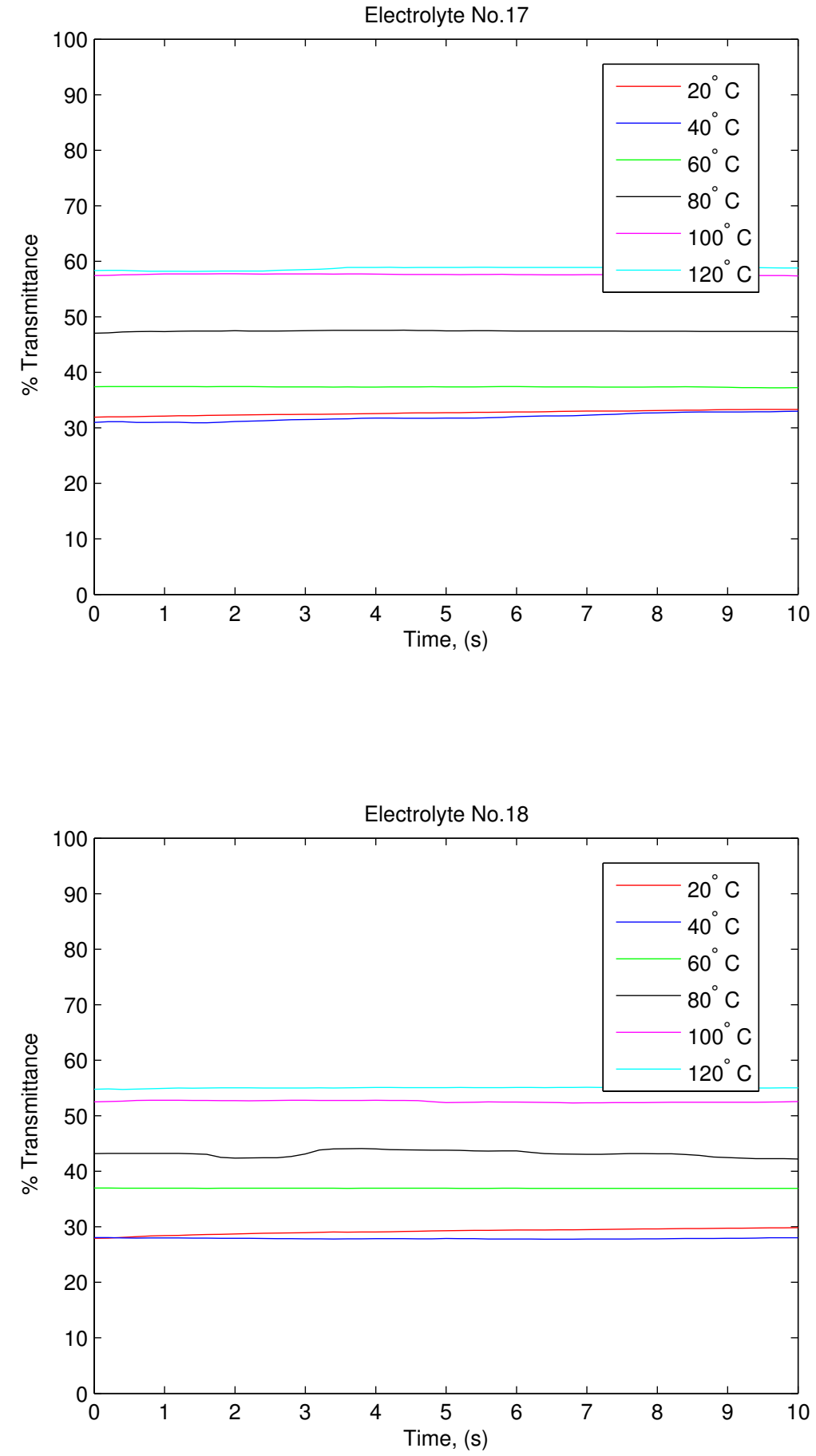
Electrolyte No.19

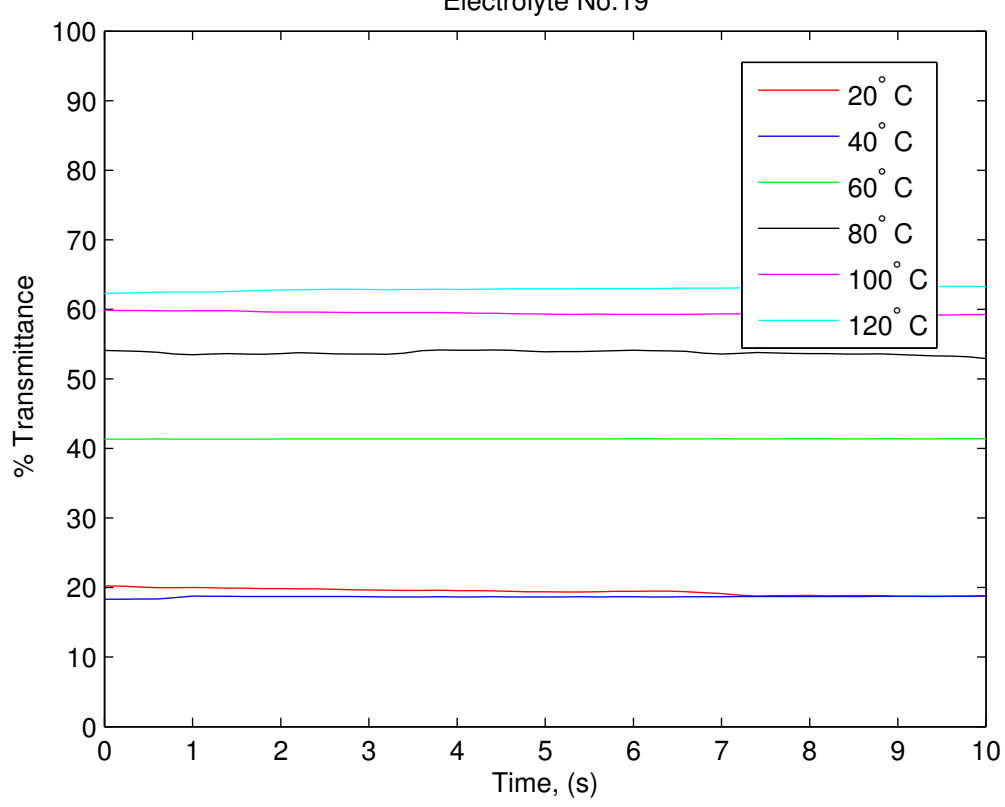

Electrolyte No.20

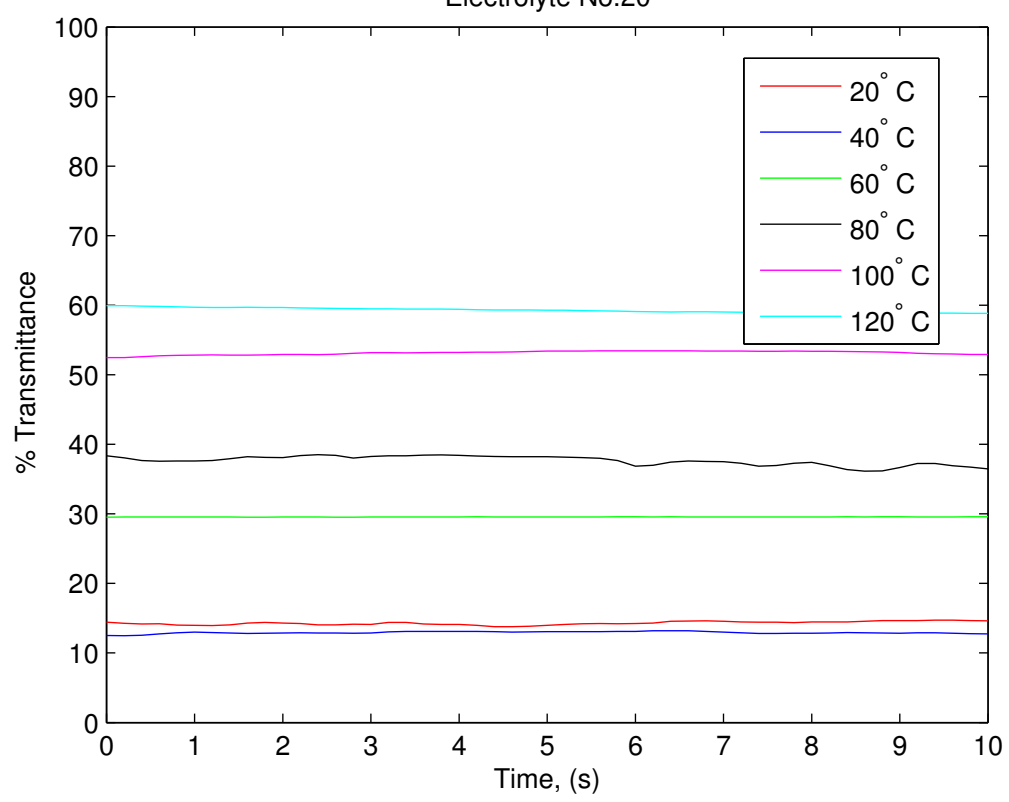


Appendix B

EXPERIMENTAL RESULTS: VARIATION OF IONIC CONDUCTIVITY OF ELECTROLYTES FROM $20^{\circ} \mathrm{C}$ TO $120^{\circ} \mathrm{C}$
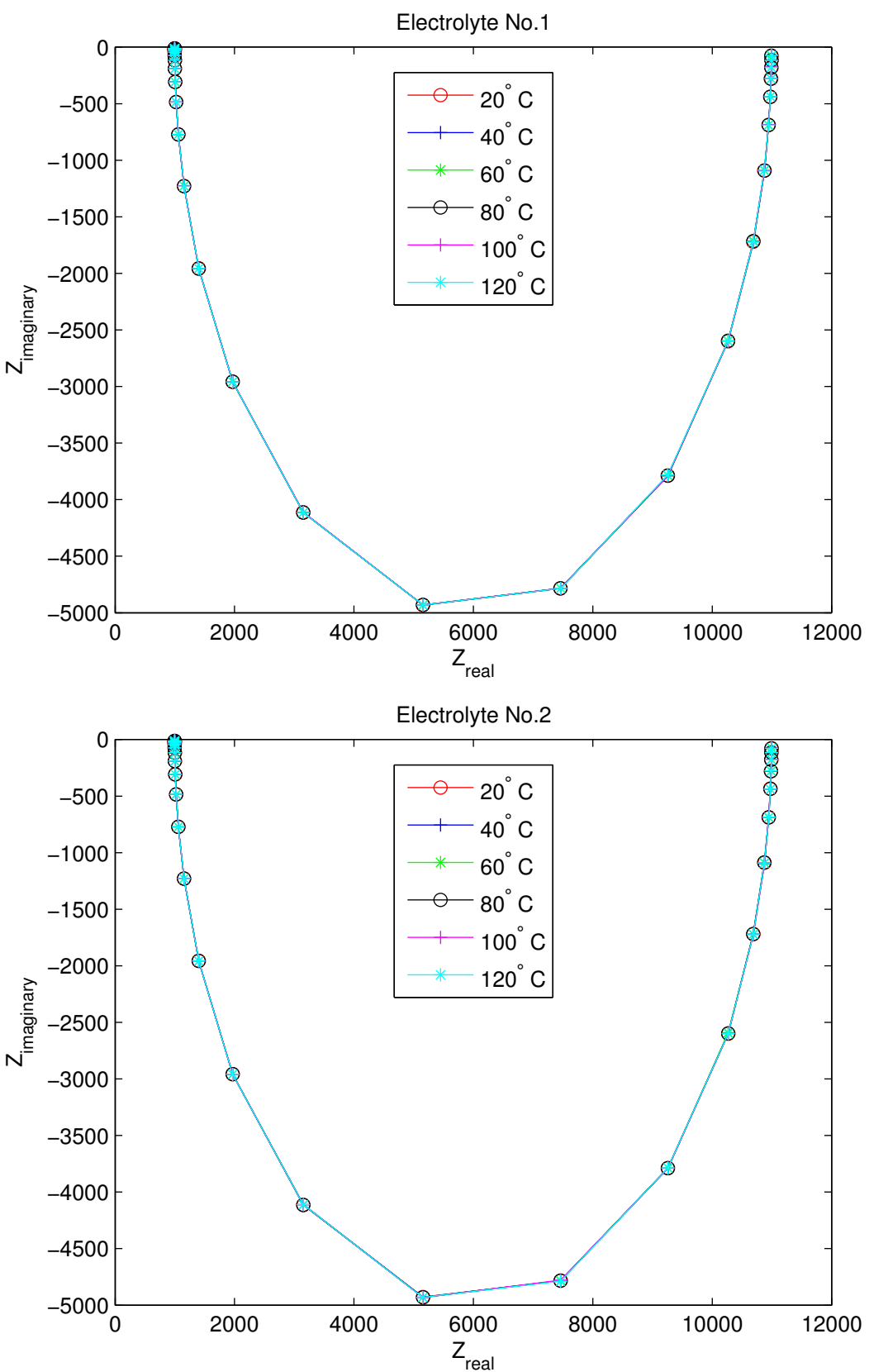

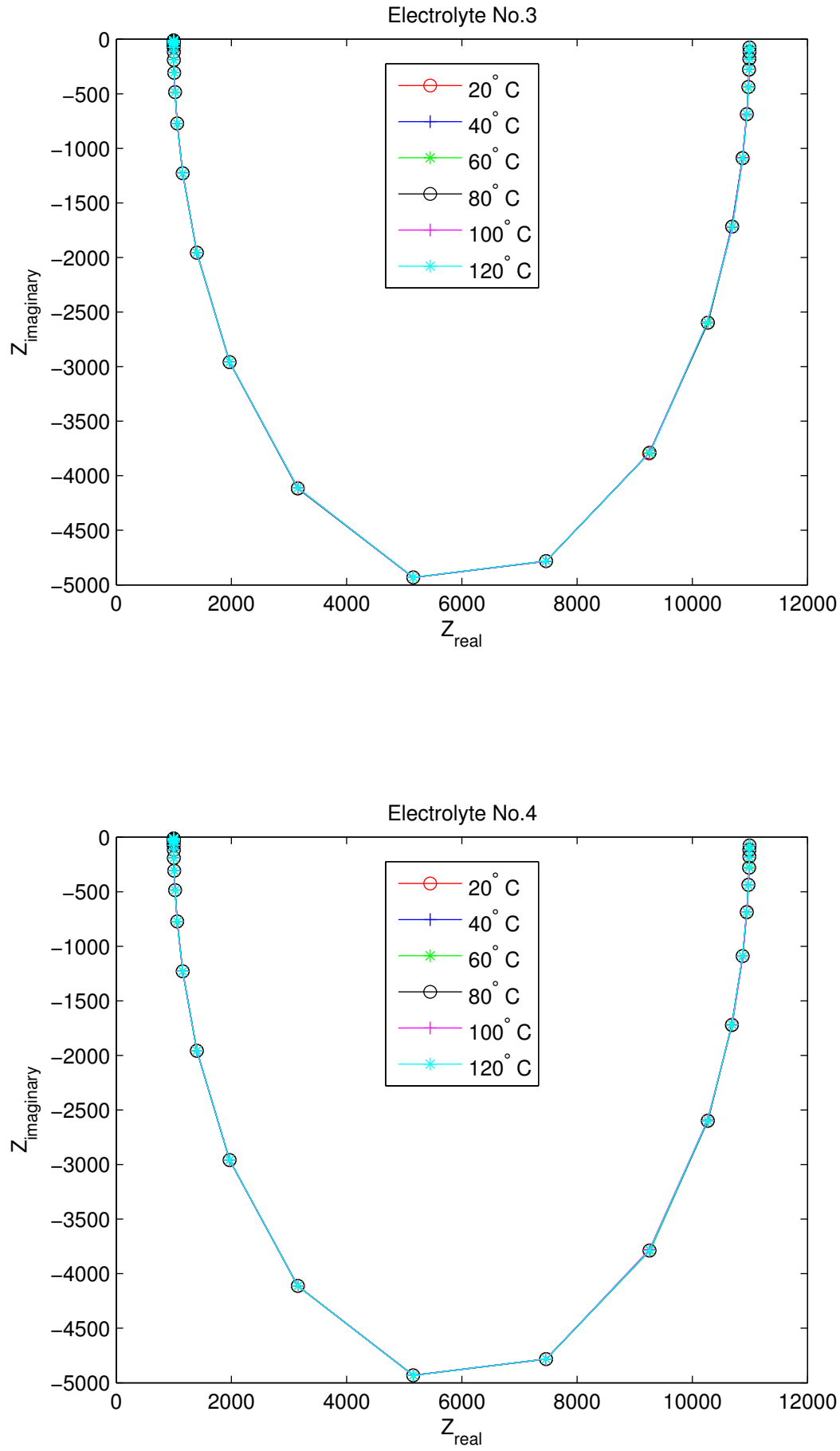

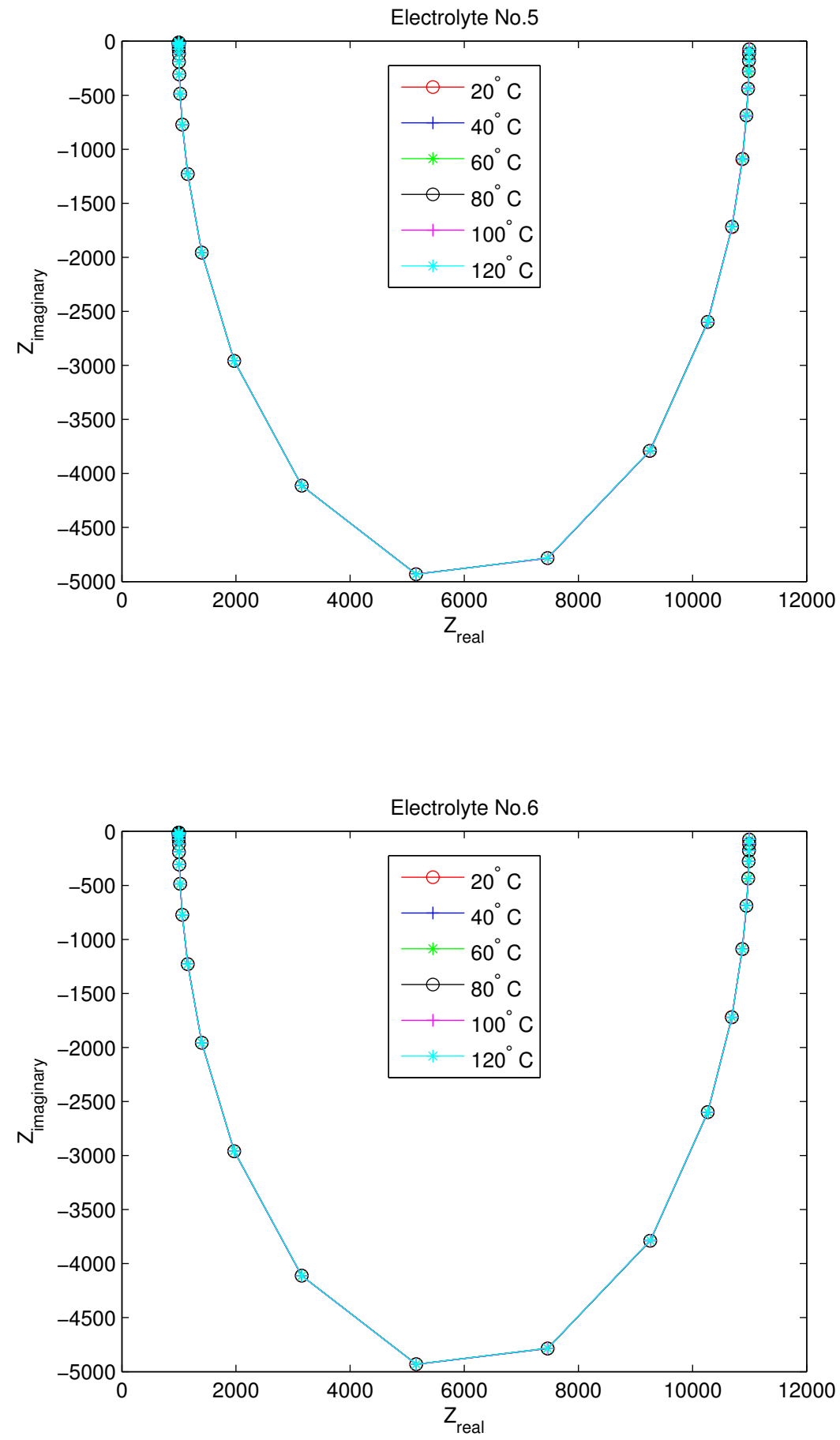

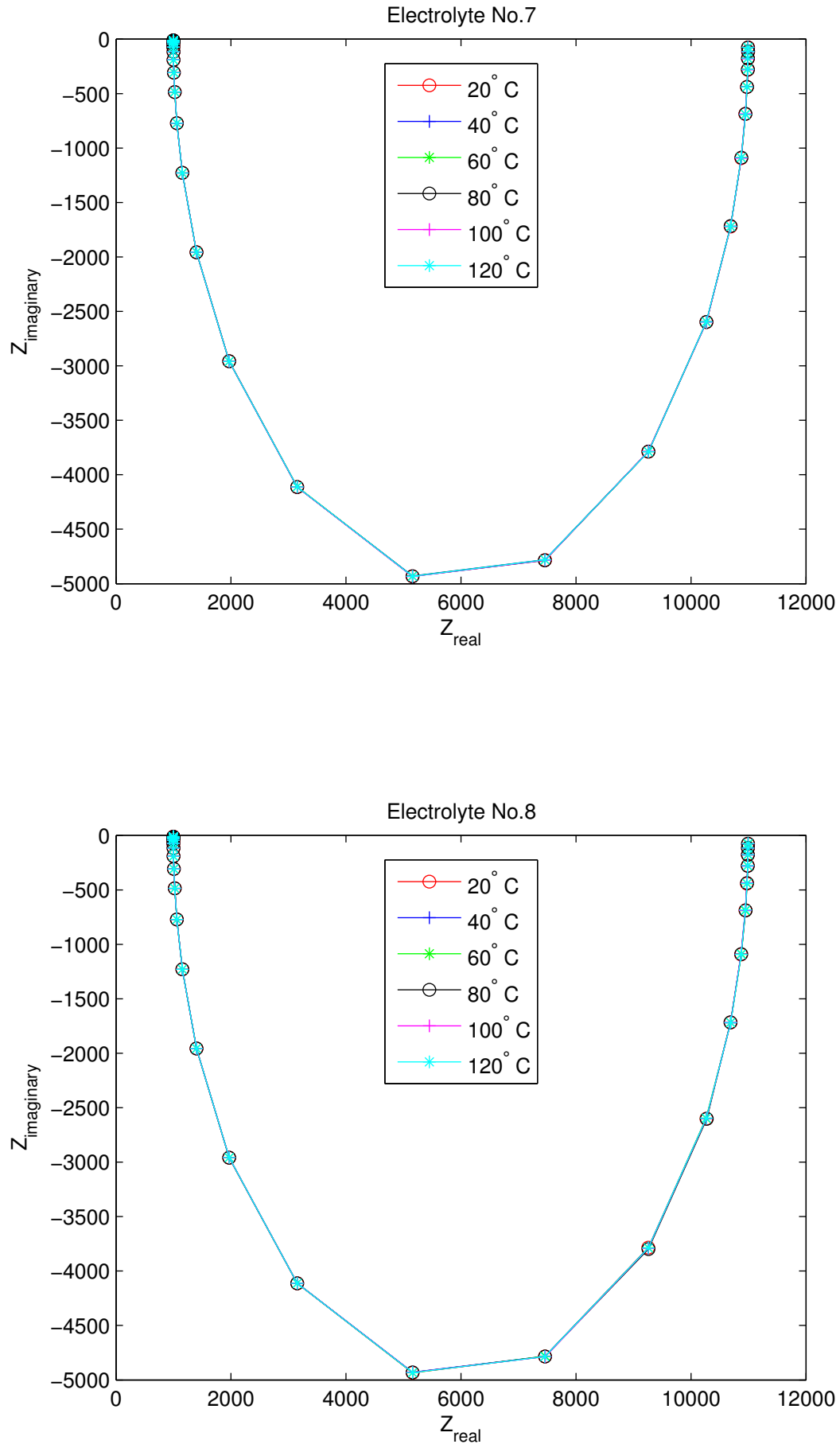

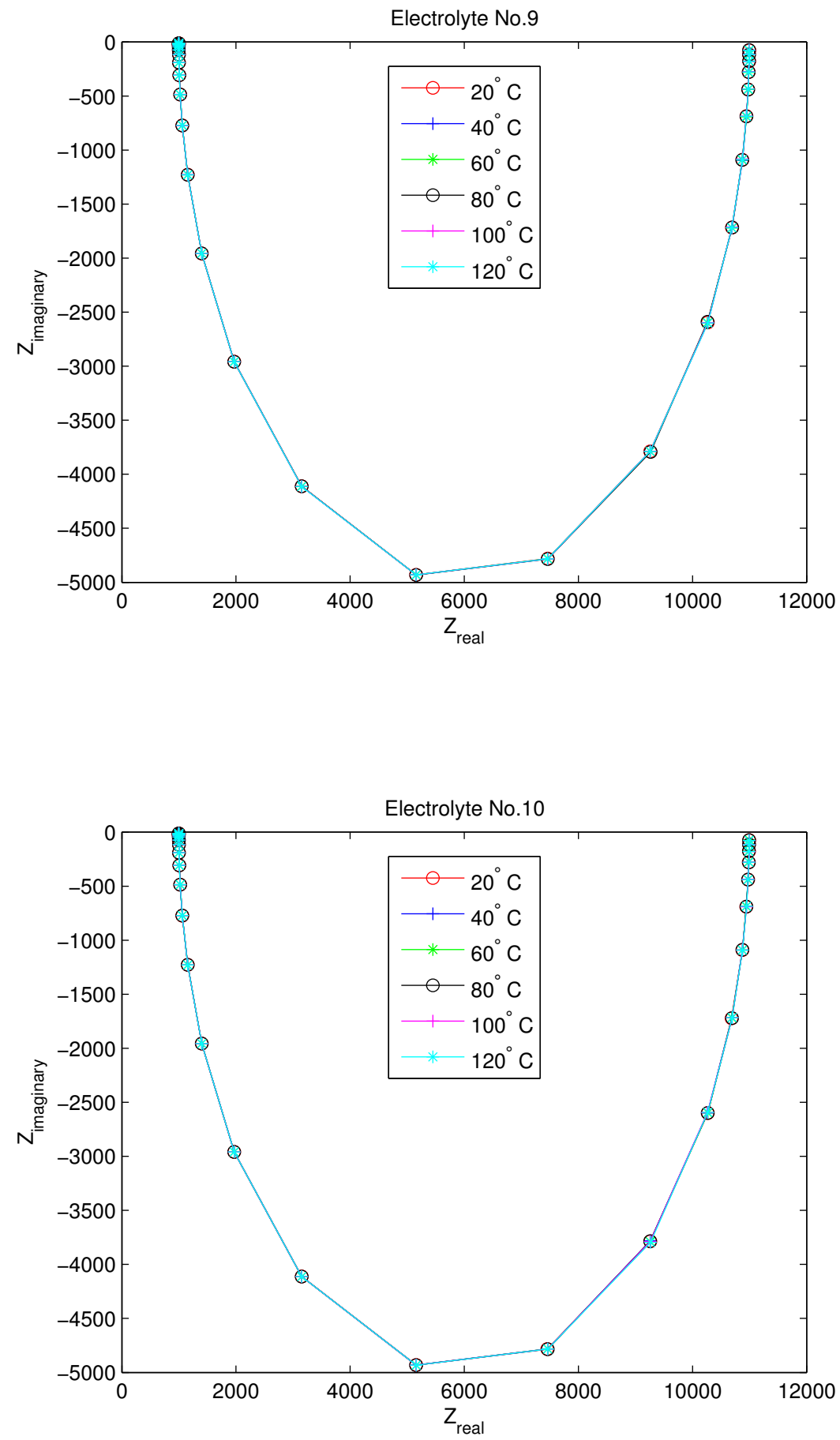

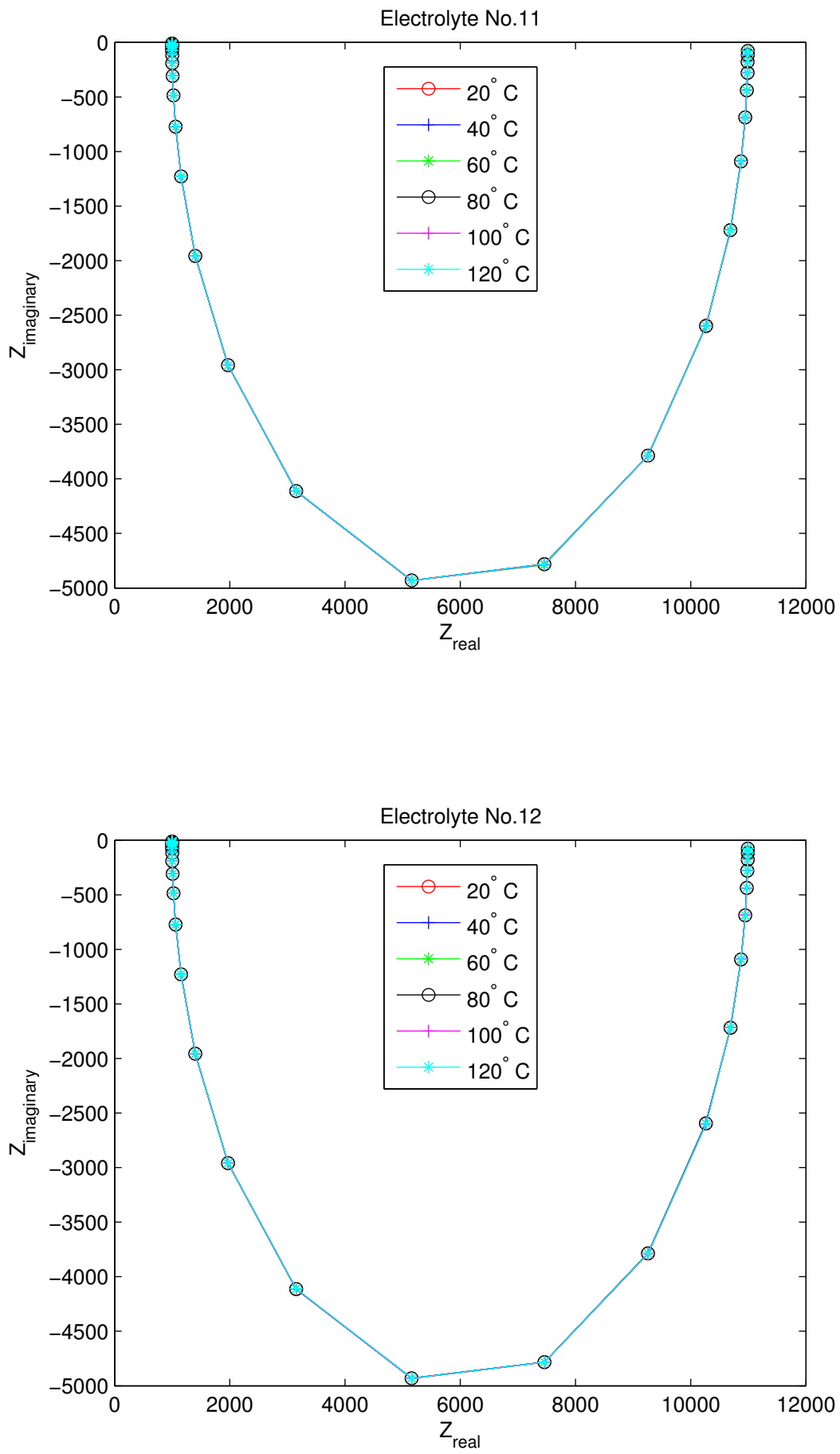
Electrolyte No.13
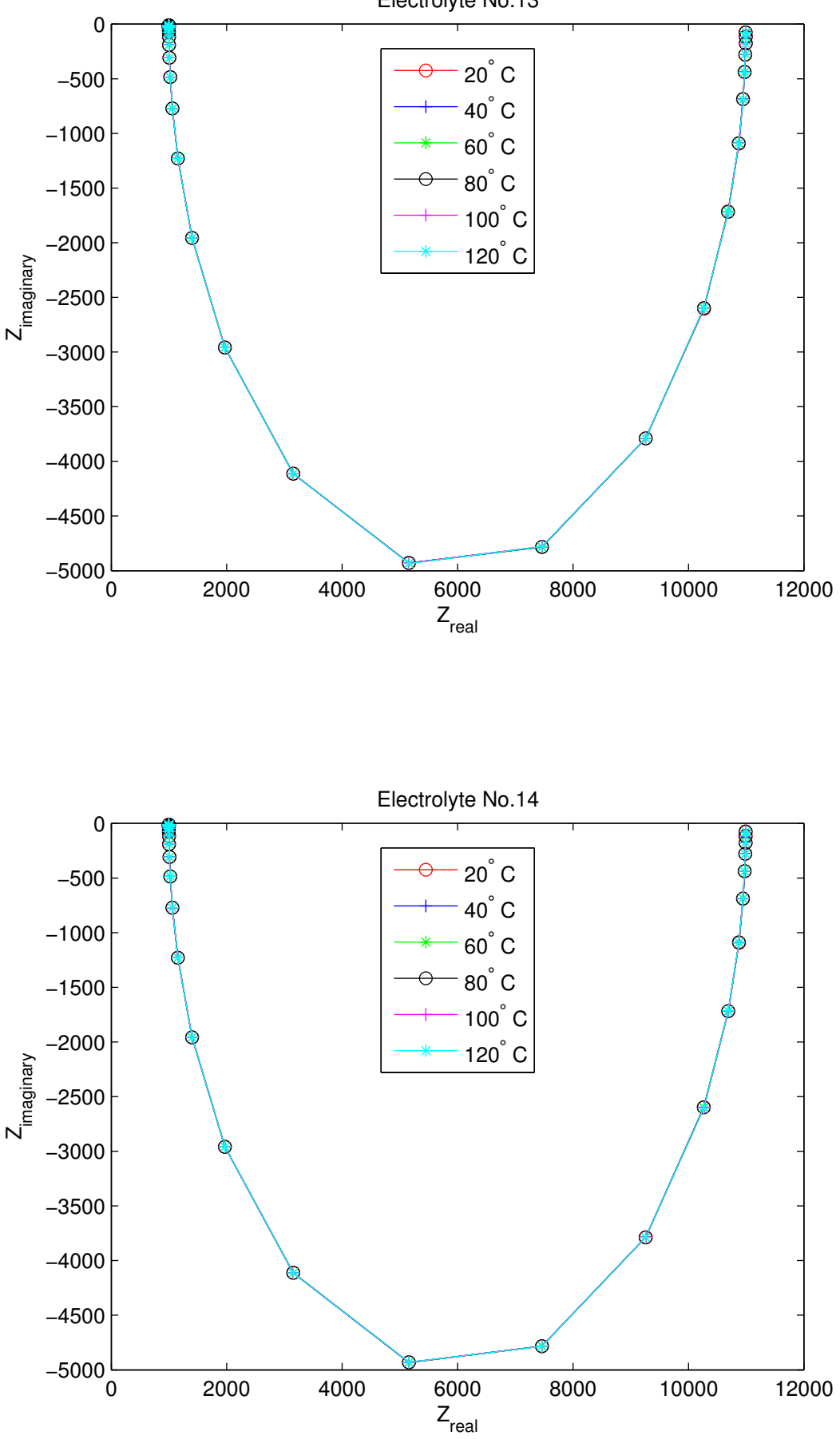

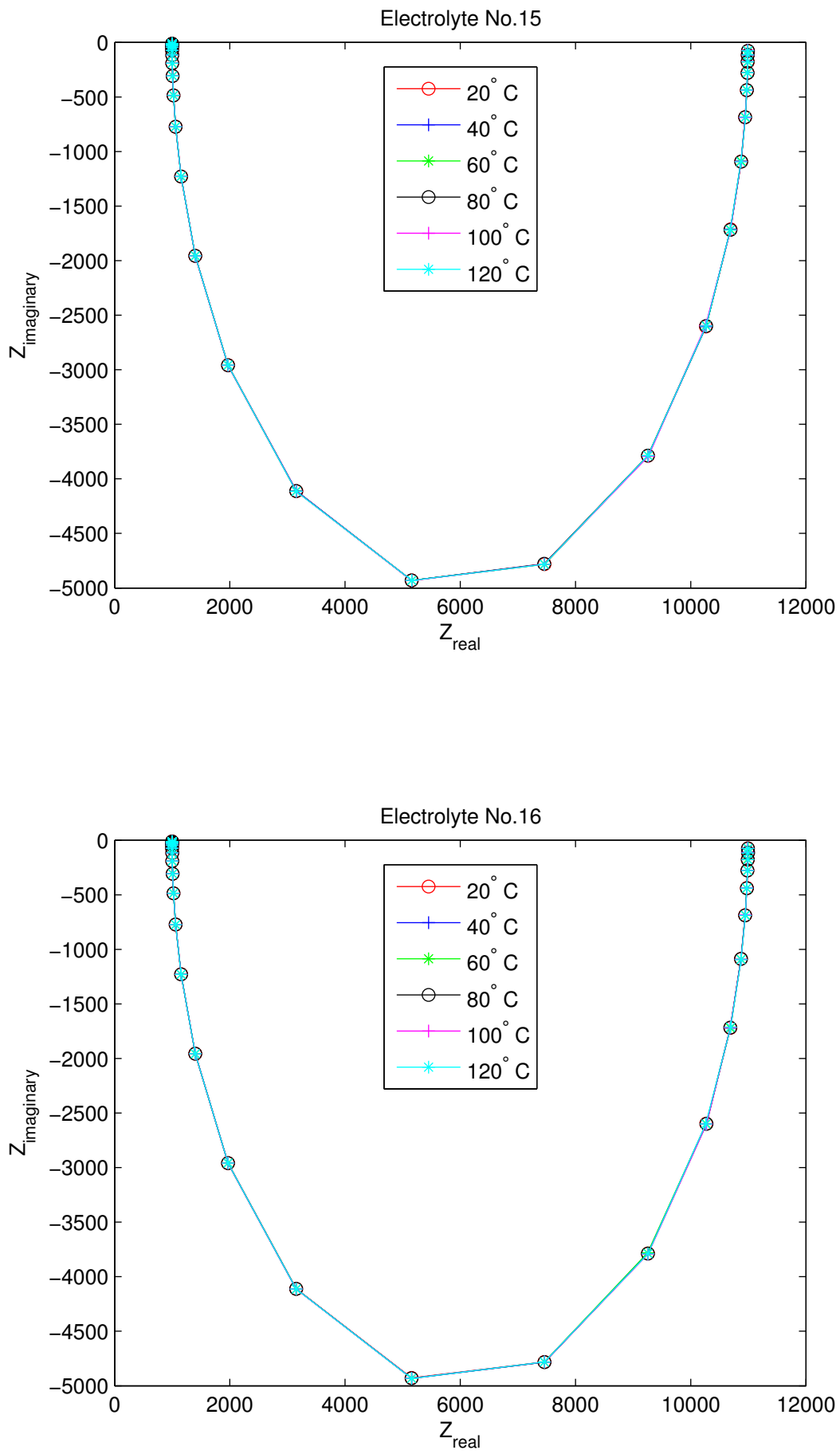
Electrolyte No.17
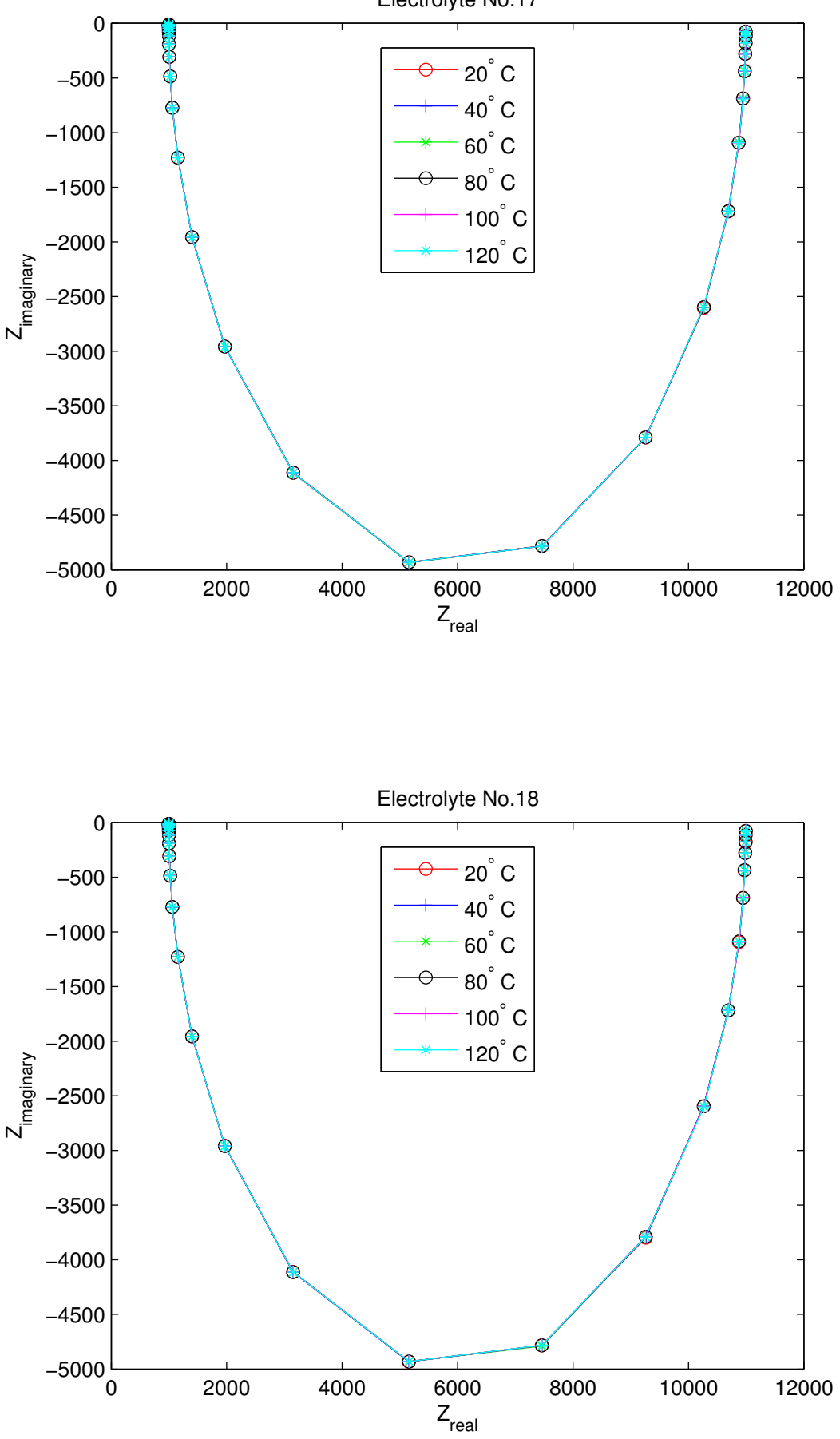

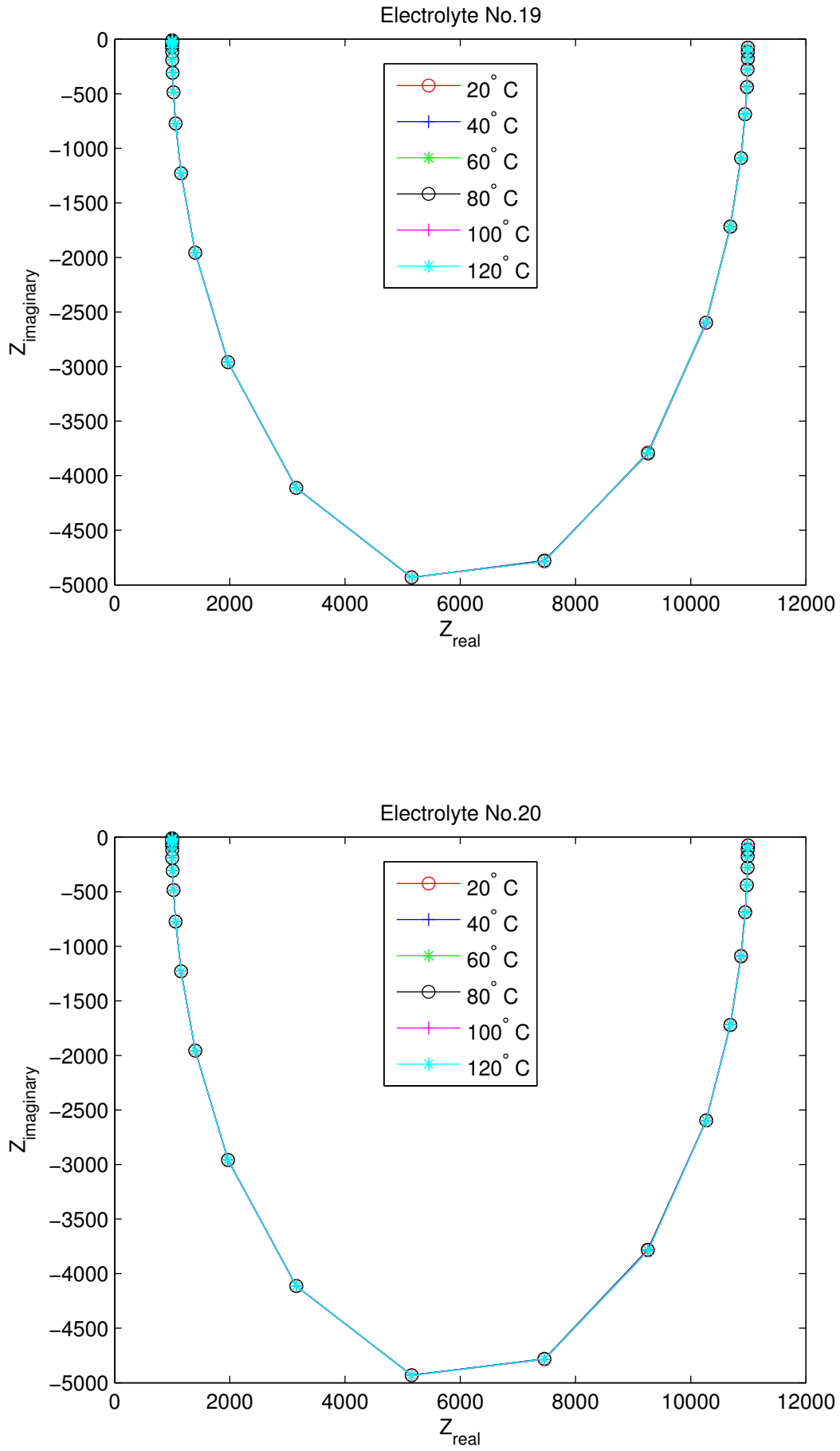
Appendix C

EXPERIMENTAL RESULTS: COUNTER ELECTRODE TRANSMITTANCE

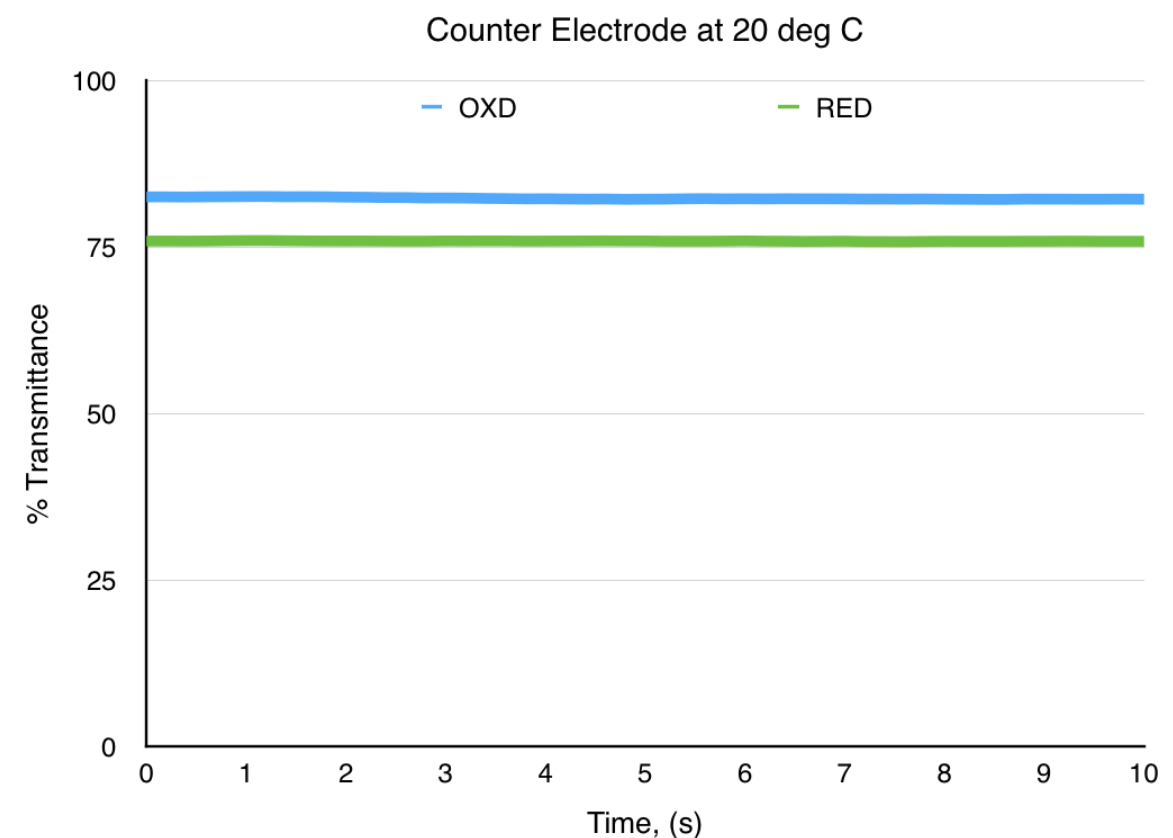

Counter Electrode at $40 \mathrm{deg} \mathrm{C}$

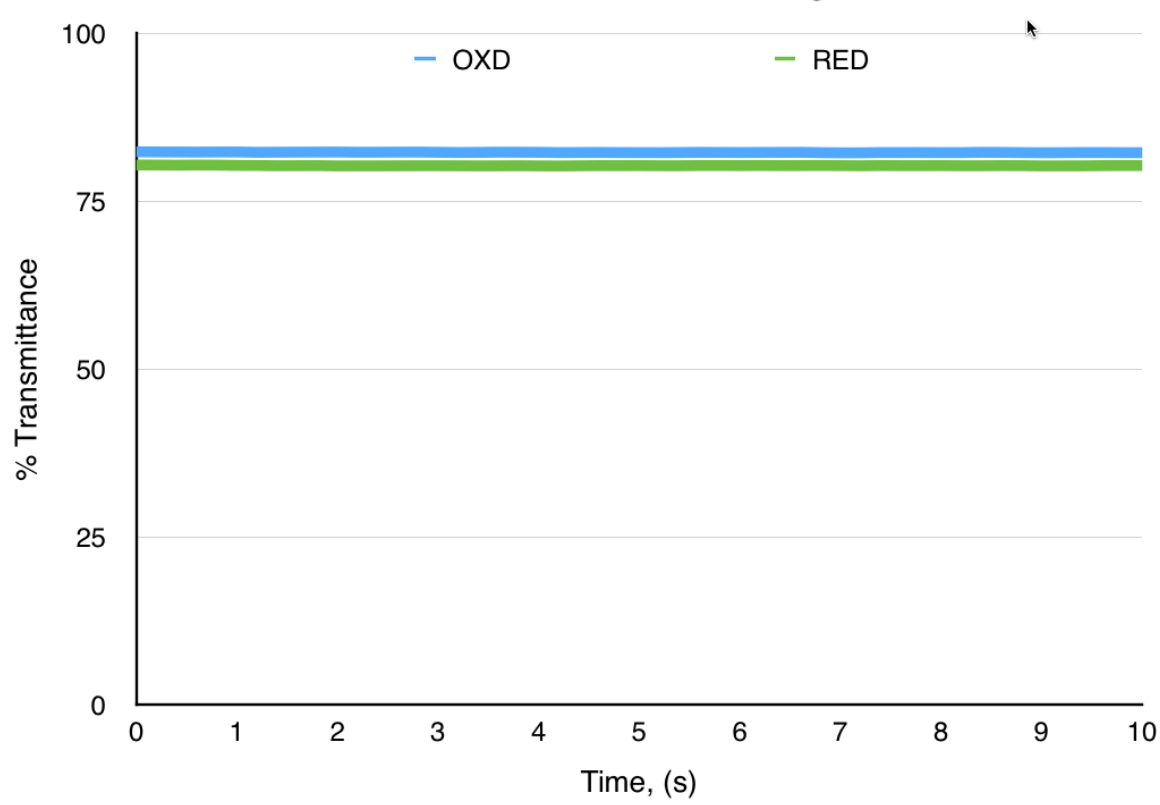



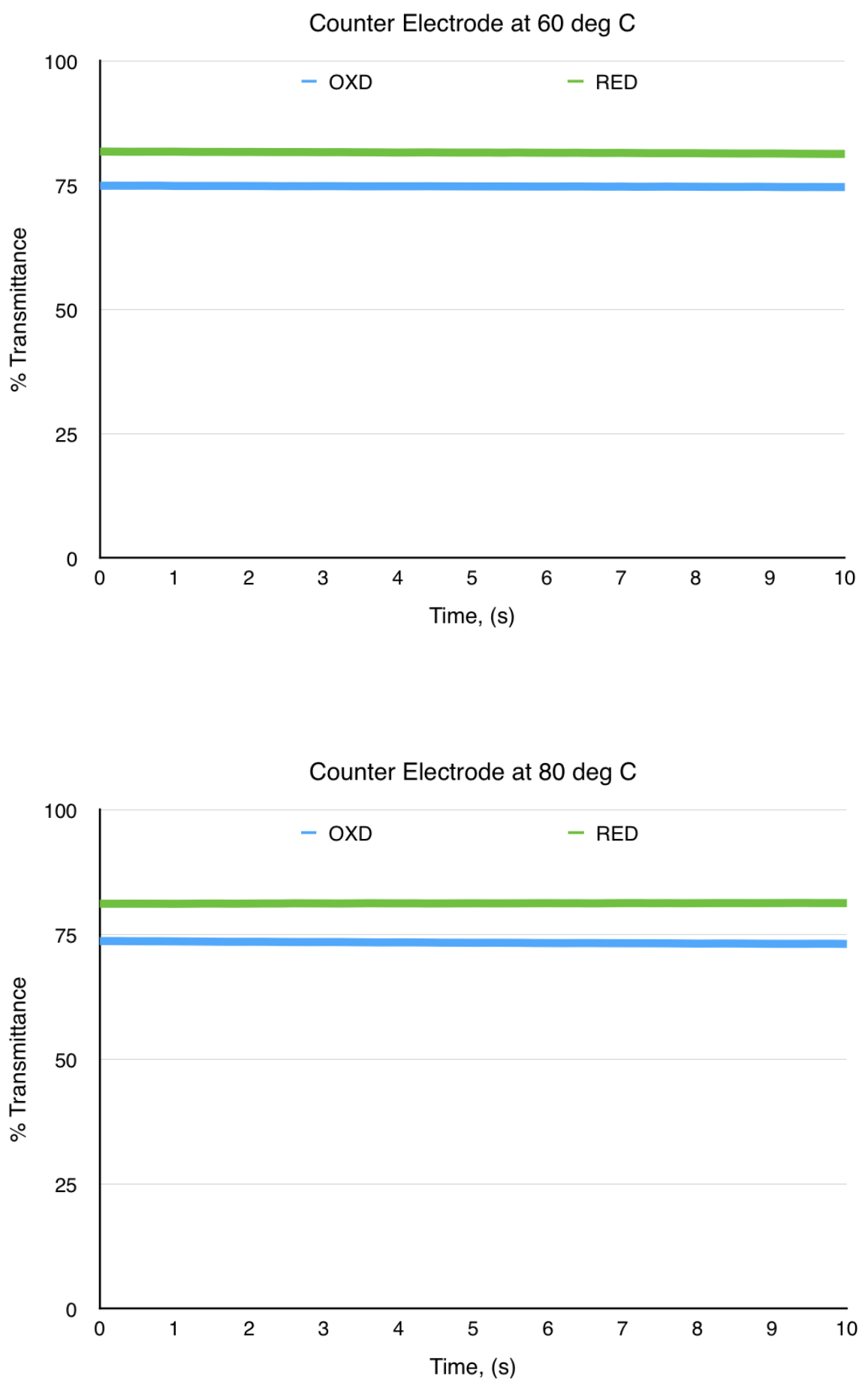

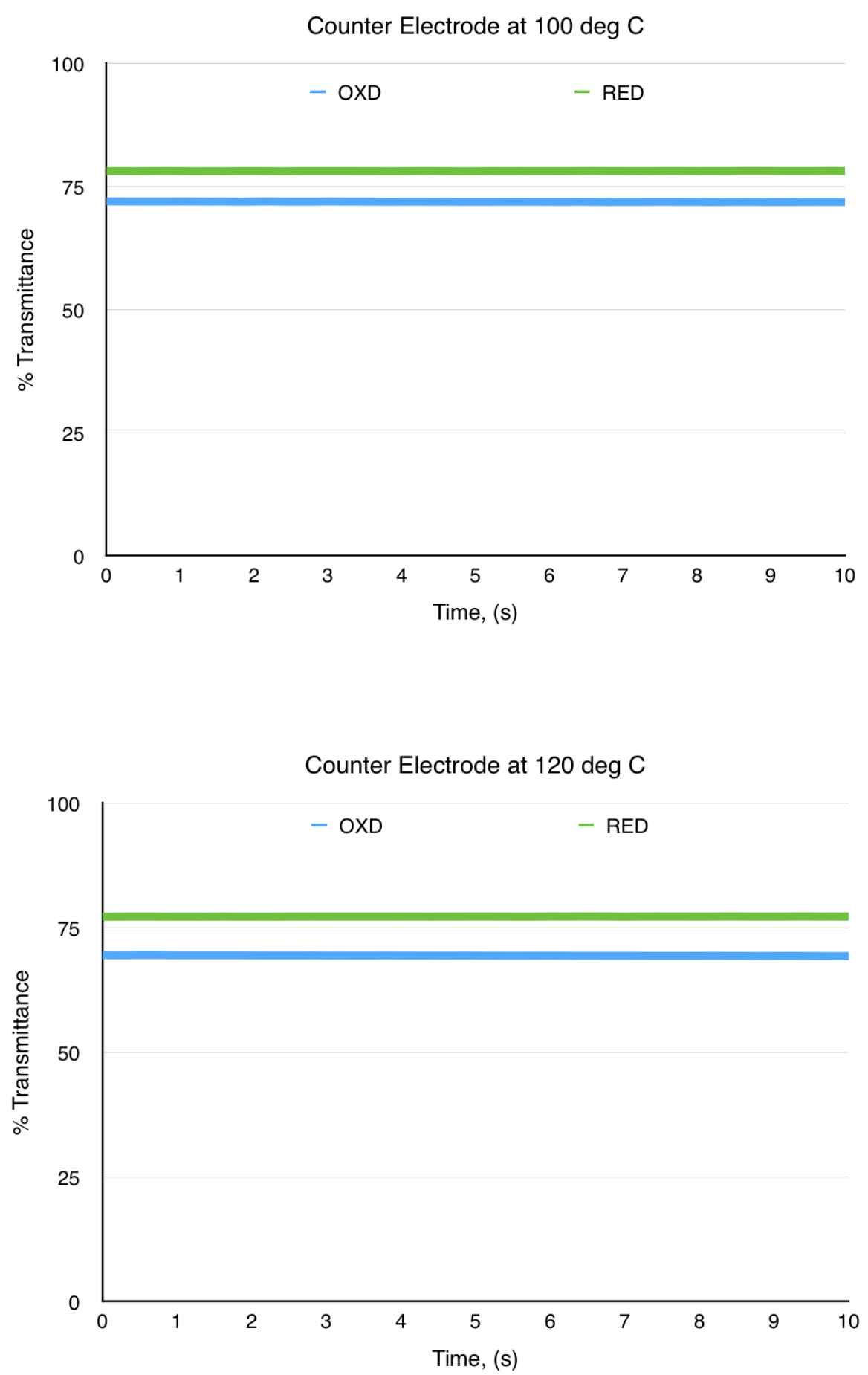
Appendix D

EXPERIMENTAL RESULTS: WORKING ELECTRODE TRANSMITTANCE
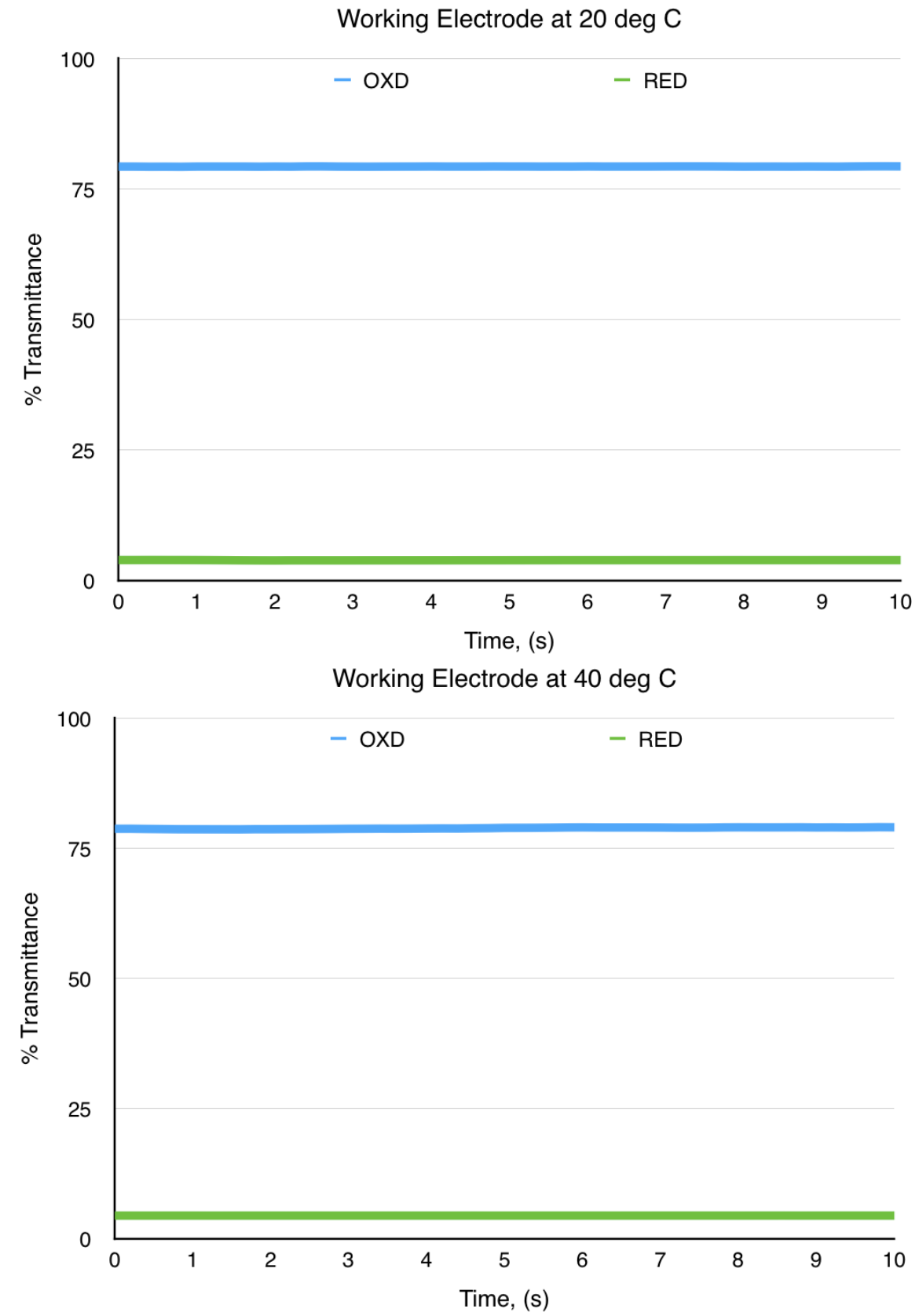

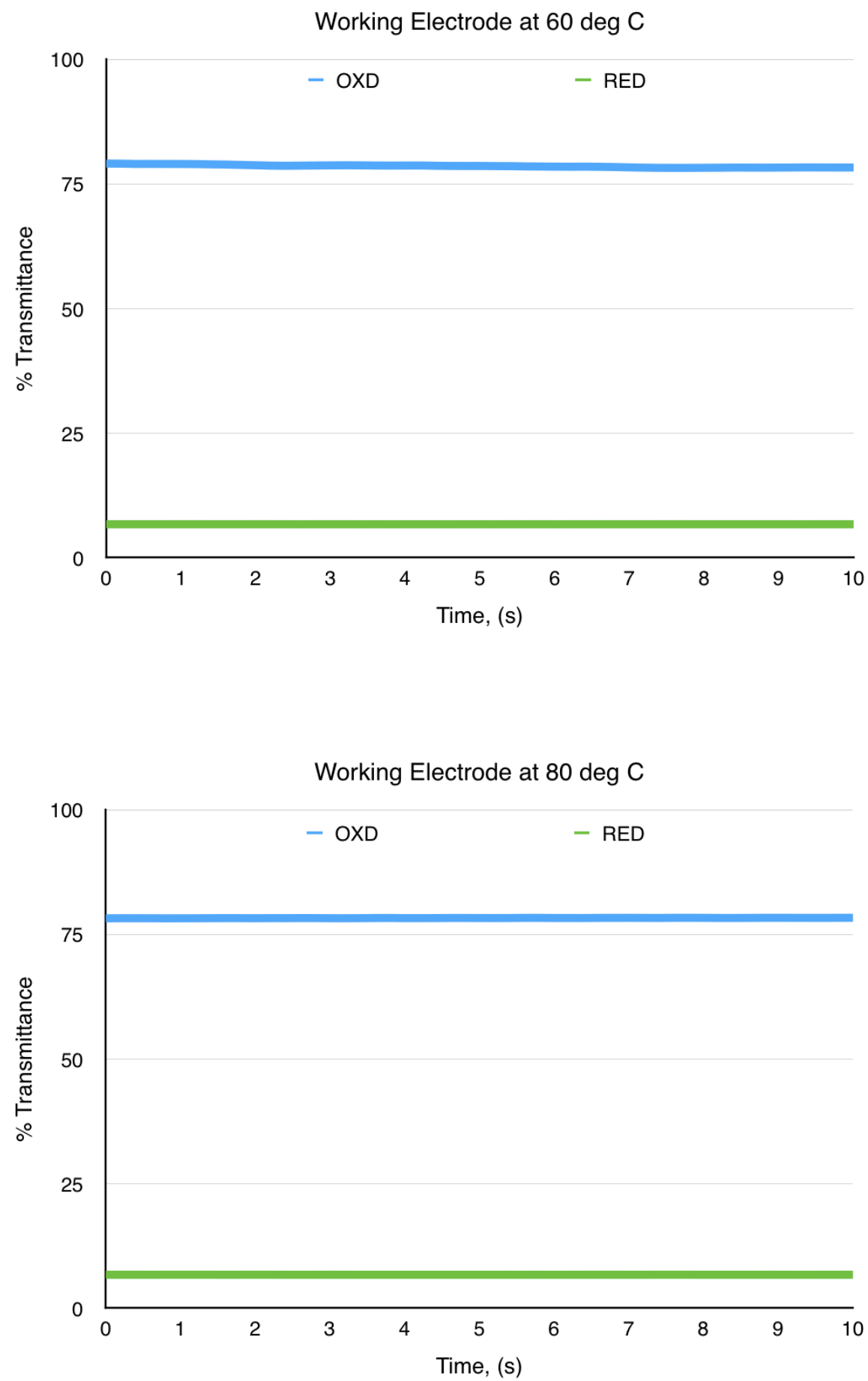

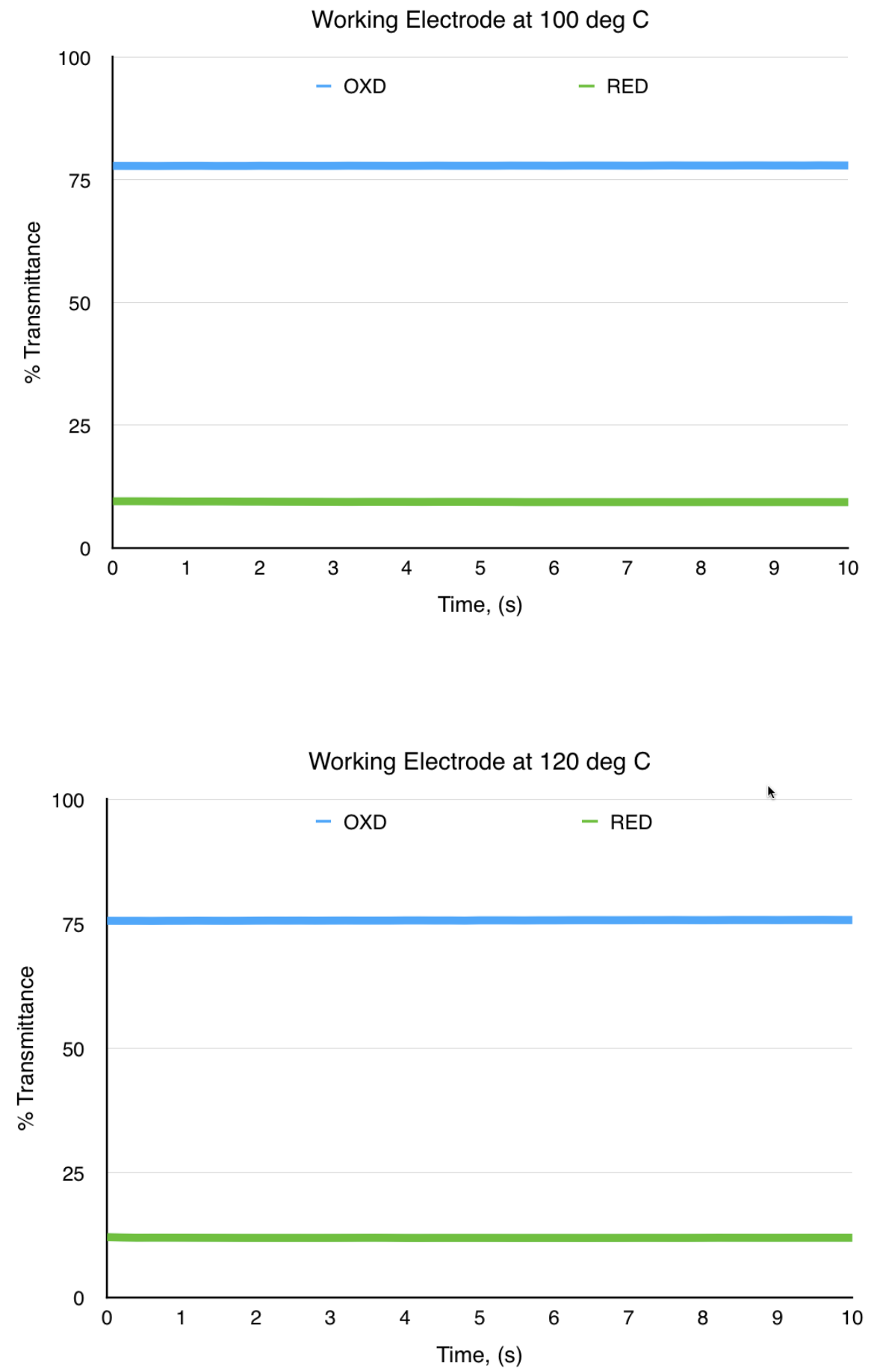